Anomalous Diffusion in Ising-like

Models 
Published by

Proefschriftmaken

phone: $+31(0) 302006189$

De Limiet 26

4131 NR Vianen

e-mail: info@Proefschriftmaken.nl http://www.Proefchriftmaken.nl

Cover illustration: The pictures on the cover are taken by Ms. Jinghan Zhang, my wife. They are taken near the Kromme Rijn river, where we use to have a walk. These photos don't have any relation to the researches contain in this thesis. However, these images express how our love for the four years' lives in Utrecht. We will miss everything there after we come back to our homeland.

ISBN: 978-94-6423-111-3

NUR: 925

Copyright (c) 2020 Wei Zhong. All rights reserved. 


\section{Anomalous Diffusion in Ising-like Models}

\section{Anomale verspreiding in Ising-achtige modellen}

(met een samenvatting in het Nederlands)

\section{Proefschrift}

ter verkrijging van de graad van doctor aan de Universiteit Utrecht op gezag van de rector magnificus, prof. dr. H.R.B.M. Kummeling, ingevolge het besluit van het college voor promoties in het openbaar te verdedigen op maandag 11 januari 2021 des ochtends te 10.30 uur

$$
\text { door }
$$

\section{Wei Zhong}

geboren op 19 september 1990 te Changting, Fujian, China 
Promotor: Prof. dr. G. T. Barkema

Copromotor: Dr. D. Panja 
This thesis is dedicated to my beloved wife Ms. Jinghan Zhang For her always accompany and endless support. 



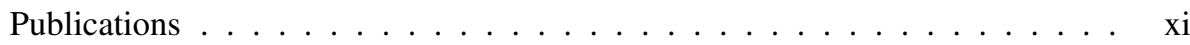

1 Introduction 1

1.1 Anomalous diffusion $\ldots \ldots \ldots \ldots \ldots \ldots \ldots$

1.2 Motivation for this thesis . . . . . . . . . . . . . . 3

1.3 Spin models considered in this thesis . . . . . . . . . . . . 4

1.3.1 Ising model and its critical properties . . . . . . . . . . 4

1.3.2 $\phi^{4}$ model and its critical properties . . . . . . . . . 5

1.3.3 Bond-diluted Ising model and its critical properties . . . . . . 6

1.4 Markov Chain Monte Carlo . . . . . . . . . . . . . . . . . . . . . . . 6

$1.4 .1 \quad$ Ergodicity . . . . . . . . . . . . . . . . . 7

1.4.2 Detailed balance . . . . . . . . . . . . . . . 7

1.5 Simulation algorithms and dynamics $\ldots \ldots \ldots \ldots$

1.5.1 Glauber dynamics . . . . . . . . . . . . . . . 8

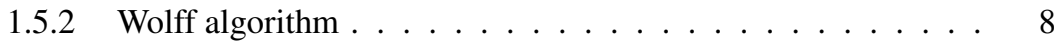

1.5 .3 Kawasaki Dynamics . . . . . . . . . . . . . . . . 11

1.6 Finite Size Scaling . . . . . . . . . . . . . . . . . . . . 11

1.7 Thesis outline . . . . . . . . . . . . . . . . . . 12

2 Generalized Langevin Equation Formulation for Anomalous Diffusion in the Ising Model at the Critical Temperature 15

2.1 Introduction . . . . . . . . . . . . . . . . . . . . . 16

2.2 Anomalous diffusion in the Ising model at the critical temperature . . 17

2.2.1 The model and dynamics . . . . . . . . . . . . . 17

2.2.2 Anomalous diffusion in the Ising model . . . . . . . . . 17

2.3 Restoring forces: the physics of anomalous diffusion in the Ising model 20

2.3 .1 Restoring forces . . . . . . . . . . . . . . . . 20

2.3.2 The time-decay behavior of restoring forces _. . . . . 20

2.3.3 Anomalous diffusion stems from these restoring forces . . . . 24 
viii

2.3.4 Restoring forces and anomalous diffusion: a similar story for polymer dynamics . . . . . . . . . . . . . . 25

2.4 Generalised Langevin Equation formulation for anomalous diffusion in the Ising model . . . . . . . . . . . . 26

2.4.1 Numerical confirmation of the GLE formulation (and determination of the damping coefficient $\zeta$ ) . . . . . . . . . 27

2.4.2 The GLE formulation for driven Ising systems _ . . . . . . 29

2.5 Discussion . . . . . . . . . . . . . . . . . . 30

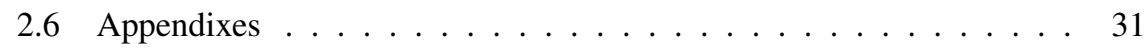

2.6.1 Appendix A: Boundary conditions effect. . . . . . . . . 31

2.6.2 Appendix B: Velocity autocorrelation of the magnetizations . 31

3 Anomalous diffusion in the off-critical Ising model with Monte Carlo spinflip dynamics

3.1 Introduction . . . . . . . . . . . . . . . . . . . 34

3.2 The model and Monte Carlo spin-flip dynamics . . . . . . . . . . . . 34

3.3 Mean-square displacement of the bulk magnetization . . . . . . . . 35

3.3.1 The effective exponent and its exponent flow diagram . . . . 35

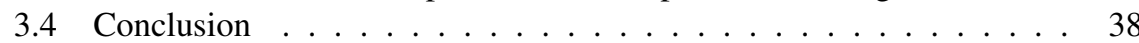

4 Critical Dynamical Exponent of the Two-Dimensional Scalar $\phi^{4}$-Model with Local Moves 41

4.1 Introduction . . . . . . . . . . . . . . . . . 42

4.2 The Measurement of the Critical Dynamical Exponent _ . . . . . . 43

4.2.1 The Model and the Dynamics . . . . . . . . . . . . . . 43

4.2.2 Measurement of the Correlation function $\langle\Phi(t) \Phi(0)\rangle \quad \ldots \quad . \quad 43$

4.2.3 Mean-Square Displacement of the Order Parameter . . . . . 44

4.3 The GLE formulation of the anomalous diffusion in the $\phi^{4}$-model . . 48

4.3.1 The GLE formulation for the anomalous diffusion in the $\phi^{4}$ model . . . . . . . . . . . . . . . 48

4.3.2 Verification of the first equation of the GLE and the power-law behavior of $\mu(t) \ldots \ldots \ldots \ldots \ldots$

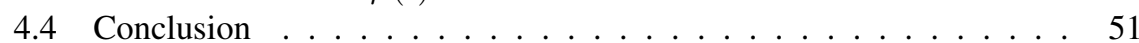

5 Super slowing down in the bond-diluted Ising model 53

5.1 Introduction . . . . . . . . . . . . . . . . . . . 54 54

5.2 Bond-diluted Ising model and its critical temperature . . . . . . . . 55

5.3 Dynamical exponent for different values of $p \ldots \ldots \ldots$

5.4 Anomalous diffusion of the total magnetization $\ldots \ldots \ldots \ldots$

5.5 Discussion . . . . . . . . . . . . . . . . . 60

5.6 Appendixes . . . . . . . . . . . . . . . 60

5.6.1 Appendix A: Relevant length scale for critical phenomena of the bond-diluted Ising model . . . . . . . . . . . . . . . 60

5.6.2 Appendix B: Equilibrium critical exponents $\nu$ and $\gamma \ldots \ldots$. . 61

5.6.3 Appendix C: Number of different types of bonds . . . . . . 62 
6 Approximate dynamical eigenmodes of the Ising model with local spinexchange moves

6.1 Introduction . . . . . . . . . . . . . . . 66

6.2 The model and the Fourier modes as the approximate dynamical modes 67

6.2.1 Ising model with local spin-exchange (Kawasaki) dynamics . 67

6.2.2 Fourier modes for line magnetization . . . . . . . . . . 68

6.2.3 Equilibrium properties of the Fourier mode amplitudes . . . . 68

6.2.4 Fourier modes as approximate dynamical eigenmodes of the model . . . . . . . . . . . . . . . 70

6.3 Dynamics of two physical observables using the Fourier modes as approximate dynamical eigenmodes . . . . . . . . . . 72

6.3.1 Autocorrelation function of the line magnetization . . . . . 72

6.3.2 Anomalous diffusion of the line magnetization . . . . . . . 73

6.4 Generalised Langevin Equation formulation for the anomalous diffusion in the Ising model with Kawasaki dynamics . . . . . . . . . . 75

6.4.1 Generalized Langevin Equation for the line magnetization . . 76

6.4.2 Verification of the power-law behavior of $\mu(t) \ldots 77$

6.5 Conclusion ......................... 77

6.6 Appendix: Scaling of $\left\langle\left|A_{p}\right|\right\rangle^{2}$ with $p$ for the 2D Ising model . . . . . . 78

7 Conclusion and outlook $\quad 83$

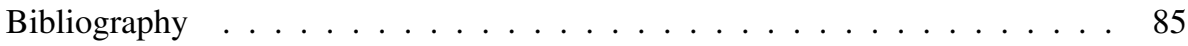

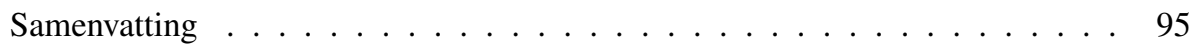

Acknowledgements . . . . . . . . . . . . . . . . . 97

Curriculum Vitae . . . . . . . . . . . . . . . . . . . 99 

This thesis is based on the following works:

- Chapter 2

Wei Zhong, Debabrata Panja, Gerard T. Barkema, and Robin C. Ball, Generalized Langevin equation formulation for anomalous diffusion in the Ising model at the critical temperature. Phys. Rev. E 98, 012124 (2018).

- Chapter 3

Wei Zhong, Gerard T. Barkema, Debabrata Panja, Anomalous diffusion in the off-critical Ising model with Monte Carlo spin-flip dynamics. in preparation.

- Chapter 4

Wei Zhong, Gerard T. Barkema, Debabrata Panja, and Robin C. Ball, Critical dynamical exponent of the two-dimensional scalar $\phi^{4}$ model with local moves. Phys. Rev. E 98, 062128 (2018).

- Chapter 5

Wei Zhong, Gerard T. Barkema, and Debabrata Panja, Super slowing down in the bond-diluted Ising model. Phys. Rev. E 102, 022132 (2020).

- Chapter 6

Wei Zhong, Debabrata Panja, and Gerard T. Barkema, Approximate dynamical eigenmodes of the Ising model with local spin-exchange moves. Phys. Rev. E 100, 012132 (2019). 



\section{CHAPTER 1}

\section{Introduction}

In this chapter, we briefly introduce some basic concepts and models of anomalous diffusion. After expressing the motivation of our research, and presenting the properties of three spin models are considered in this thesis, the Monte Carlo methods used in this thesis are discussed. This is followed by a description of the Metropolis algorithm, the Wolff algorithm, and Kawasaki dynamics. Then, we also show how finite-size scaling can be used to extract critical exponents from the data obtained from Monte Carlo simulations. At the end of this chapter, the outline of the thesis is provided. 


\subsection{Anomalous diffusion}

In case of normal diffusion the mean-square displacement (MSD) of a particle $\left\langle\Delta r^{2}(t)\right\rangle$ increases linearly in time. The term anomalous diffusion is used to denote a particle's mean-square displacement $\left\langle\Delta r^{2}(t)\right\rangle$ deviating from (its normal behavior of) increasing linearly in time $t$; and commonly refers to the power-law behavior $\left\langle\Delta r^{2}(t)\right\rangle \sim t^{c}$ for some $c \neq 1$. The cases of $c<1$ and $c>1$ are known as subdiffusion and superdiffusion respectively.

Although the term "anomalous" diffusion was originally coined to denote an anomaly — in this case, a deviation from normal diffusion — anomalous diffusion has increasingly become the norm [1]. Observed in many materials and systems such as in fractal systems and disordered media [2, 3], financial markets [4], transport in (crowded) cellular interiors [5], and migration of cells [6], bacteria [7], and animal foraging [8], anomalous diffusion has naturally received intense attention in the last decade. Interest in the topic revolves largely around the following questions. What causes the exponent to differ from unity? Can one predict the exponent from the underlying dynamics of the system? Are there universality classes for systems exhibiting anomalous diffusion?

A number of distinct classes of stochastic processes have been developed/identified for anomalous diffusion in the recent years. Three most prominent theoretical (stochastic) models of anomalous diffusion are:

- Transport on fractals: a popular model used for percolating and disordered materials $[3,9,10,11]$, wherein the moving particle encounters obstacles on its path,

- Continuous-time random walk (CTRW): a model where particles move from trap to trap $[12,13,14,15]$, where times of waiting at the traps as well as the trap-to-trap distance is power-law distributed, and

- Gaussian models like fractional Brownian motion (fBm) which describes a Gaussian process with power-law memory $[16,17]$, attributed to the "material medium" that surrounds the particle that undergoes anomalous diffusion.

Despite the progress as mentioned above, which model describes an instance of (experimentally) observed anomalous diffusion is often the subject of fierce debate, as evidenced by the recent case of anomalous diffusion observed for tracer particles in cell cytoplasm [18, 19, 20, 21, 22, 23], where all three of the above stochastic models have been fitted to the experimental data $[19,20,21,22,23,24,25,26]$. For physical systems where the dynamical rules for particle movement are known (in contrast to a complicated medium like a cell cytoplasm), one would expect to have a much easier task to model anomalous diffusion, yet it can still remain quite a challenge.

An overview of the available theoretical models, including a summary of their distinctive features and stochastic properties can be found in a recent perspective article [27]. 


\subsection{Motivation for this thesis}

Recently, Walter and Barkema [28] reported numerically that the magnetization of the $2 D$ Ising model at the critical temperature experiences anomalous diffusion with anomalous exponent equal to $\gamma /(\nu z) \approx 0.81$ (Fig. 1.1), where $\gamma=1.75$ and $\nu=1.0$ are two equilibrium critical exponents for the $2 D$ Ising model, and $z \approx 2.1665$ [29] is the dynamical exponent. Their qualitative explanation for this subdiffusive behavior (note $\gamma /(\nu z)<1$ ) is as follows.

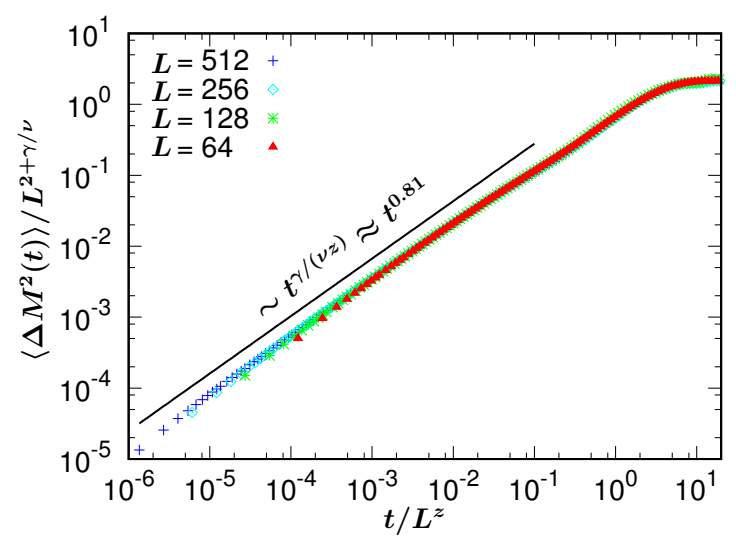

Figure 1.1: The mean-square displacement (MSD) of the total magnetization in the $2 D$ Ising model at $T_{c}$. The solid line indicates that the total magnetization experiences anomalous diffusion with the anomalous exponent $c \approx \gamma /(\nu z)$.

The mean-square displacement (MSD) of the total magnetization $M(t)$ is defined as

$$
\left\langle\Delta M^{2}(t)\right\rangle=\left\langle[M(t)-M(0)]^{2}\right\rangle .
$$

At the short times $(t \approx 1)$, the individual spin flips in the model are uncorrelated, and since there are $L^{D}$ spins all together in the $D$-dimensional Ising model,

$$
\left\langle\Delta M^{2}(t)\right\rangle \sim L^{D} t
$$

At long times $\left(t \gtrsim L^{z}\right)$, the magnetization is no longer auto-correlated, i.e., $\langle M(t) M(0)\rangle \approx$ 0 , meaning that

$$
\left\langle\Delta M^{2}(t)\right\rangle=\left\langle 2 M(t)^{2}\right\rangle \sim L^{D+\gamma / \nu} .
$$

If the two points $\left\langle\Delta M^{2}(1)\right\rangle \approx\left(1, L^{D}\right)$ and $\left\langle\Delta M^{2}\left(L^{z}\right)\right\rangle \approx\left(L^{z}, L^{D+\gamma / \nu}\right)$ in a $\log -\log$ plot are joined by a straight line, then the line will have a slope of $\gamma /(\nu z)$, which is exactly the subdiffusion exponent seen in Fig. 1.1. However, this qualitative explanation does not provide an insight into the underlying physics of the anomalous diffusion in the Ising model.

The central theme of this thesis is the subdiffusive behavior of magnetization in a number of spin models at their critical temperatures (inclusive of the Ising model). We 
establish that the subdiffusive behavior from "restoring forces", works as "inertia" of the surrounding spins to resist changes in magnetization. The restoring forces give rise to to memory effects, slowing down the MSD of the total magnetization, and thereby leading to the subdiffusive behavior.

The restoring forces can be measured - as we do for all the spin models considered in this thesis - by tracking the "velocity" autocorrelation function of magnetization $\langle\dot{M}(t) \dot{M}(0)\rangle$. As we do so, we find that $\langle\dot{M}(t) \dot{M}(0)\rangle \sim-L^{D} t^{(c-2)}$ (note the negative sign, due to the restoring force). Mathematically, integrating this quantity twice in time using the Green-Kubo relation one obtains

$$
\left\langle\Delta M(t)^{2}\right\rangle \sim L^{D} t^{c}
$$

This physics of anomalous diffusion of magnetization is consistent with a Generalized Langevin Equation (GLE) description with power-law memory effects in time, i.e., the fBm description of anomalous diffusion. Emphatically, the negative sign in the velocity autocorrelation function in Eq. (1.4) rules out a CTRW description for anomalous diffusion in these spin models.

The work in this thesis also suggests that anomalous diffusion of magnetization in spin models likely generically belongs to the fBm class.

\subsection{Spin models considered in this thesis}

We have considered three spin models here, i.e., the normal Ising model, the scalar $\phi^{4}$ model, and the bond-diluted Ising model in two- and (or) three-dimensions with computer simulations. Periodic boundary conditions are employed. Now, let's get into more details about different models.

\subsubsection{Ising model and its critical properties}

As one of the most important and well known models to study phase transition, the Ising model describes the evolving dynamics of $N$ spins on the lattice. The Hamiltonian of the Ising model on an $L \times L$ square lattice, without an external field, is defined as

$$
\mathcal{H}=-J \sum_{\langle i, j\rangle} s_{i} s_{j}
$$

where $s_{i}= \pm 1$ is the spin at site $i$, and $J$ is the coupling constant of interaction among the spins. The summation runs over all the nearest-neighbor spins.

It has been proved by Ernst Ising that there is no phase transition for the $1 D$ Ising model [30]. However, for the spatial dimension $d \geq 2$ the transition does exist, and Onsager solved the $2 D$ Ising model analytically [31].

When the temperature is approaching the critical temperature, the correlation length diverges, a number of critical exponents are proposed to understand the critical behavior of the system, and some of them are listed in Tab. 1.1. 


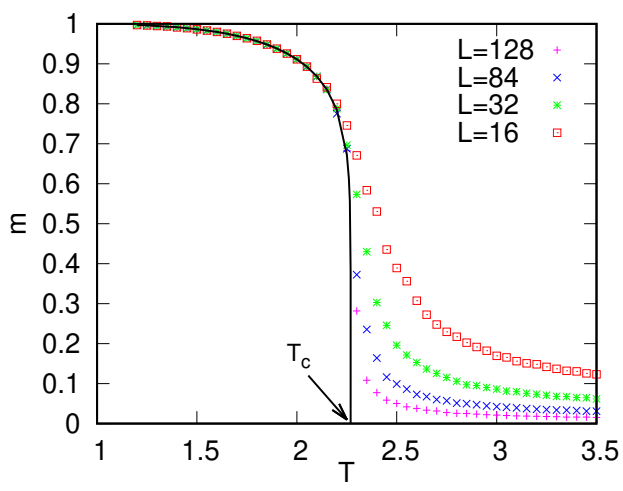

Figure 1.2: Phase diagram for the $2 D$ Ising model without the external field. The solid line is the analytic result calculated by Onsager [31], and for the rest results are from Monte Carlo simulations. As we can see from the figure that when the system size becomes large, the behavior of the order parameter $m$ is approaching to the analytic result.

\begin{tabular}{c|c|c|c|c}
\hline \hline $\begin{array}{c}\text { Ising model } \\
\text { dimension } d\end{array}$ & $\gamma$ & $\nu$ & $z_{c}$ & $T_{c}$ \\
\hline \hline 2 & $7 / 4$ & 1 & $2.1665(12)$ & $\frac{2}{\ln (1+\sqrt{2})}$ \\
\hline 3 & $1.237075(10)$ & $0.629971(4)$ & $2.03(4)$ & $4.5116174(2)$ \\
\hline
\end{tabular}

Table 1.1: Some critical exponents and the critical temperature in the Ising model [32], using $k_{B}=J=1$ for the critical temperature $T_{c}$.

\subsection{2 $\phi^{4}$ model and its critical properties}

The $\phi^{4}$-model has become one of the most useful tools in studying of critical phenomena $[33,34,35,36]$. In two dimensions, the lattice version of the $\phi^{4}$-model is defined by the action $\mathcal{S}$ and Hamiltonian $\mathcal{H}$ as

$$
\mathcal{S}=\frac{\mathcal{H}}{k_{B} T}=-\beta \sum_{\langle i j\rangle} \phi_{i} \phi_{j}+\sum_{i}\left[\phi_{i}^{2}+\lambda\left(\phi_{i}^{2}-1\right)^{2}\right],
$$

where $-\infty<\phi_{i}<\infty$ is the dynamical variable at site $i, \beta$ and $\lambda$ are two model constants. The summation of the first term in the r.h.s of Eq. (1.6) runs over all the nearest-neighbor spins, and for an $L \times L$ square lattice $0 \leq(i, j)<L$. The order parameter for the $\phi^{4}$-model is defined as $\Phi=\sum_{i} \phi_{i}$, and the dynamics of the model is given by $[37,38]$

$$
\begin{aligned}
\dot{\phi}_{i} & =-\Omega \frac{\partial \mathcal{S}}{\partial \phi_{i}}+\xi(i, t) \\
\left\langle\xi(i, t) \xi\left(i^{\prime}, t^{\prime}\right)\right\rangle & =2 \Omega \delta\left(i-i^{\prime}\right) \delta\left(t-t^{\prime}\right),
\end{aligned}
$$


where $\xi(i, t)$ is a Gaussian noise term, and $\Omega$ represents the dissipation constant, which is related to the noise term by the Fluctuation-Dissipation relation (1.8).

The equilibrium properties of the model in relation to the critical phenomenon are well-studied. Earlier investigations of the 2D and 3D lattice $\phi^{4}$-model have indicated that the critical exponents $\gamma$ and $\nu$ are the same as these for the Ising model, e.g. in 2D, $\gamma=1.75$ and $\nu=1[39,40,41]$. Simultaneously, Monte Carlo simulations of the 2D lattice $\phi^{4}$-model have supported the idea that the $\phi^{4}$-model belongs to the Ising universality class [42], although its dynamical properties are not well studied.

\subsubsection{Bond-diluted Ising model and its critical properties}

As a variation of the Ising model, the Hamiltonian of the bond-diluted Ising model without an external field is defined as

$$
\mathcal{H}=-\sum_{\langle i j\rangle} J_{i j} s_{i} s_{j},
$$

where $s_{i}= \pm 1$ is the spin residing at site $i,\langle i j\rangle$ denotes the sum running over all nearest neighbor sites, and the coupling constant $J_{i j}$ is given by the distribution function

$$
P\left(J_{i j}\right)=p \delta\left(J_{i j}-1\right)+(1-p) \delta\left(J_{i j}\right),
$$

with $p$ being the bond concentration $(0 \leq p \leq 1)$. The function (1.10) simply means that the value of $J_{i j}$ is 1 with probability $p$, and 0 otherwise.

For the normal Ising model $(p=1)$, there is a second-order phase transition at $T_{c}(1)=2 / \ln (1+\sqrt{2})$ [32]. When $p$ reaches the percolation threshold $p_{c}=1 / 2$, its critical temperature decreases to zero: $T_{c}\left(p_{c}\right)=0$ [43]. We have shown in Chapter 5 that for the $2 D$ bond-diluted Ising model, the equilibrium exponents $\gamma$ and $\nu$ are numerically indistinguishable respectively from $7 / 4$ and unity for $p \geq 0.6$, confirming the results from Ref. [44].

\subsection{Markov Chain Monte Carlo}

To study the equilibrium properties of models like the Ising model, a commonly used technique is Markov Chain Monte Carlo.

We generate a Markov chain as

$$
C_{0}, C_{1}, C_{2}, \ldots . . C_{n} \text {. }
$$

The probability for configuration $C_{t+1}$ depends only on the previous configuration $C_{t}$. The probability of getting to $C_{t+1}$ from $C_{t}$ is via the transition probability

$$
P\left(C_{t} \rightarrow C_{t+1}\right),
$$

where the transition probability $P\left(C_{t} \rightarrow C_{t+1}\right)$ should not vary over time, and it depends only on the states $C_{t}$ and $C_{t+1}$. Besides, it should obey the following constrain that 


$$
\sum_{C_{t}} P\left(C_{t} \rightarrow C_{t+1}\right)=1
$$

In order to generate states that are sampled according to the Boltzmann distribution, it is sufficient to have algorithms that satisfy the conditions of "ergodicity" and "detailed balance".

\subsubsection{Ergodicity}

An important property of Monte Carlo algorithms is ergodicity. It means that given a long enough time of simulation, the system should be able to reach any state of the system from any other state.

As mentioned before, in our Monte Carlo simulations, we want to sample the configurations according to the Boltzmann distribution. If the algorithm we have employed violates ergodicity, then there is at least a state $v$ that can not be reached from another state $u$ no matter how long we have run our simulation. When the state $u$ is chosen as the initial state, then the Boltzmann probability will not hold because of the zero probability to find the state $v$. Therefore, ergodicity should not be violated for the algorithms that we have used, so that we can measure the correct average values of the quantities we are interested in.

\subsubsection{Detailed balance}

When our Monte Carlo method meets the condition of ergodicity, the system can reach any state from any other state. Another important condition is detailed balance. Together, ergodicity and detailed balance ensure that when the system reaches equilibrium, the probability distribution of the configurations is indeed the Boltzmann distribution.

Mathematically the condition of detailed balance can be defined as [45]

$$
p_{v} P(v \rightarrow u)=p_{u} P(u \rightarrow v) .
$$

This condition indicates that on average the frequency of transitions from state $u$ to $v$ is the same as the frequency of transitions from $v$ to $u$. When the system is equilibrated, it guarantees that the probability distribution of the configurations is the Boltzmann distribution, which reveals that $p_{v}=\exp \left(-\beta E_{v}\right)$ and $p_{u}=\exp \left(-\beta E_{u}\right)$, resulting in

$$
\frac{P(u \rightarrow v)}{P(v \rightarrow u)}=\frac{p_{v}}{p_{u}}=e^{-\beta\left(E_{v}-E_{u}\right)} .
$$

This equation, together with Eq. (1.13), are the constraints for the transition probability in our algorithms.

Next, we will discuss some well known algorithms and dynamics. First, we discuss Glauber dynamics, in which single spins are proposed to be flipped, and the proposed spin-flips are accepted according to the Metropolis algorithm. Next, we discuss a cluster algorithm named Wolff algorithm. Thirdly, we discuss Kawasaki dynamics in which spins of neighboring sites are exchanged. 


\subsection{Simulation algorithms and dynamics}

\subsubsection{Glauber dynamics}

The first algorithm we need to explore is the Metropolis algorithm, also known as Metropolis-Hastings algorithm.It was first proposed by Nicholas Metropolis and his coauthors in 1953 [46]. It was used as a fast algorithm to sample atomistic states of hard-sphere gases. Later in 1970 [47], Hastings extended this algorithm to a more general formula; this extended algorithm is called the Metropolis-Hastings algorithm.

The algorithm describes that in the Ising model with the so-called Glauber dynamics, which is a single-spin-flip dynamics, i.e., at each time step, only a single spin will be selected to attempt a flip. The transition probability between two state $u$ and $v$ is

$$
P(u \rightarrow v)= \begin{cases}e^{-\beta\left(E_{v}-E_{u}\right)} & \text { if } E_{v}-E_{u}>0 \\ 1 & \text { otherwise }\end{cases}
$$

Supposing we have two states $u$ and $v$, and $E_{v}-E_{u}>0$, then we have

$$
\frac{P(u \rightarrow v)}{P(v \rightarrow u)}=\frac{e^{-\beta\left(E_{v}-E_{u}\right)}}{1}=e^{-\beta\left(E_{v}-E_{u}\right)} .
$$

We can obtain the same result as Eq. (1.17) for $E_{v}-E_{u}<0$. It proves that the Metropolis probability Eq. (1.16) satisfies detailed balance.

For Ising-like models, in practice we implement the Metropolis algorithm by using the following steps:

1 Initialize. One commonly-used initial state is obtained by assigning arbitrary values to each spin. Another commonly-used initial state is obtained by taking all spins up (or down).

2 For each iteration $t$, a single spin is randomly selected to flip, yielding a transition from state $u$ to state $v$. The resulting energy difference $\Delta E=E_{v}-E_{u}$ is measured. If $\Delta E<0$, then the flip is accepted. Otherwise, the flip is accepted with the usual Metropolis probability $e^{-\beta \Delta E}$.

Normally, a thermalization process is necessary for all simulations so that we can measure different quantities after the system has reached its equilibrium state. The single-spin-flip dynamics with Metropolis probability is known as Glauber dynamics.

\subsubsection{Wolff algorithm}

Glauber dynamics is often already a fast algorithm. However, we are mostly interested in the physics near the critical point, and due to the phenomenon of critical slowing down [45], exactly at the critical point, the dynamics is not very fast. Often, the correlation time $\tau$ at the critical point increases in power-law fashion with the system size $L$ :

$$
\tau \sim L^{z}
$$




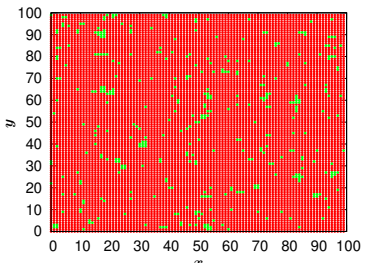

(a)

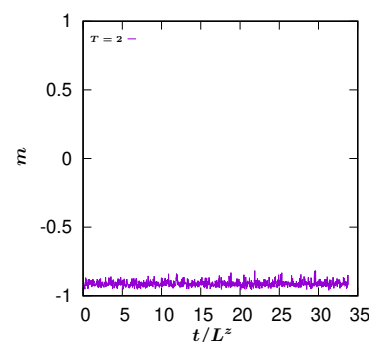

(d)

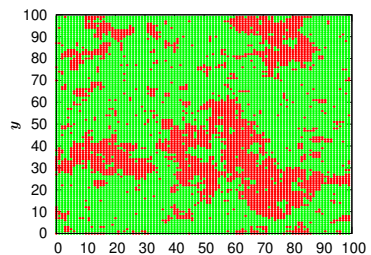

(b) ${ }^{x}$

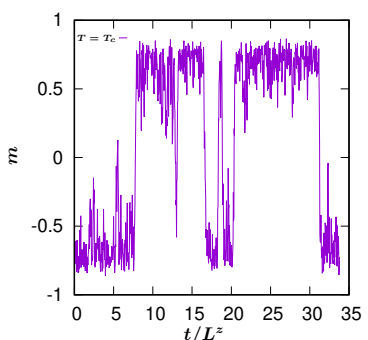

(e)

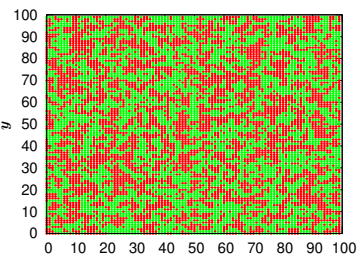

(c) ${ }^{x}$

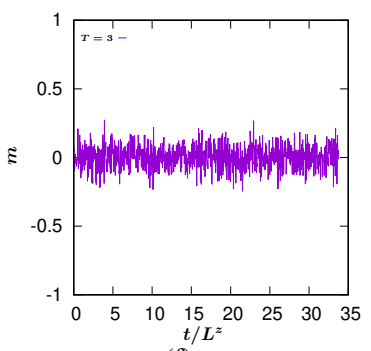

(f)

Figure 1.3: (a)-(c) Typical snapshots obtained via the Metropolis algorithm for the $2 D$ Ising model with the temperatures are $T=2.0, T_{c}$, and 3.0, respectively. The green dots represent value +1 of the spin, and the red ones are for spins whose value is -1 . The system size is $L=100$ for each figure. (d)-(f) The corresponding time series of the order parameter $m$ after the system reaches its equilibrium state. 
The dynamical exponent of the Metropolis algorithm is $z=2.1665$ (12) [29], which indicates that this algorithm is still a bit slow at $T_{c}$. It inspires us to look for a new algorithm for simulating faster dynamics around the critical point, and the Wolff algorithm is one of the candidate solutions.

The Wolff algorithm, which was proposed by Ulli Wolff in 1989 [48], is a cluster algorithm. It is a relatively small extension of previous work of Swendsen and Wang [49] which flips multiple clusters in a single iteration. In contrast, the Wolff algorithm only flips one single cluster at each time step. When we are in state $u$, at first, a seed spin is selected randomly. Then, we begin to check its neighbors. Those neighbor spins that share the same value with the seed spin, are added to the cluster with probability $P_{a d d}$. For each new spin that was added to the cluster, we also check its neighbors and test them in the same way to find out whether we should add these neighbors to the cluster or not. We repeat this procedure until there are no spins left in the cluster whose neighbors have not been considered to join the cluster. Finally, the cluster is ready, and we flip all spins in the cluster, leading us to the new state $v$.

About the probability $P_{a d d}$, a convenience choice is

$$
P_{a d d}=1-\exp (-2 \beta J) .
$$

Since the transition probability can be separated as

$$
P(u \rightarrow v)=g(u \rightarrow v) A(u \rightarrow v),
$$

where $g(u \rightarrow v)$ is the selection probability, which defines the probability that given an initial state $u$, our algorithm will generate the target state $v$, and $A(u \rightarrow v)$ is the acceptance ratio.

For the Wolff algorithm, if there are $m$ attempts to add a new spin to the cluster are failed when we go from state $u$ to $v$, and there are $n$ failures from state $v$ to $u$, then the selection probabilities are $\left(1-P_{a d d}\right)^{m}$ and $\left(1-P_{a d d}\right)^{n}$. Then we have

$$
\frac{P(u \rightarrow v)}{P(v \rightarrow u)}=\frac{g(u \rightarrow v) A(u \rightarrow v)}{g(v \rightarrow u) A(v \rightarrow u)}=\left(1-P_{a d d}\right)^{m-n} \frac{A(u \rightarrow v)}{A(v \rightarrow u)}
$$

The acceptance ratios are chosen as $A(u \rightarrow v)=A(v \rightarrow u)=1$, and the energy difference of state $u$ and $v$ depends on the failure cases. For each of the failure cases from $u$ to $v$, the bond to be broken is shared by two aligned spins, resulting in the energy change $+2 J$. From state $v$ to $u$, it experiences a similar process. So, if we focus on the energy change between state $v$ and state $u$, then we have

$$
E_{v}-E_{u}=2 J m+(-2 J n)=2 J(m-n) .
$$

Combining Eqs.(1.20) - (1.22) we have

$$
\frac{P(u \rightarrow v)}{P(v \rightarrow u)}=e^{-2 \beta J(m-n)}=e^{-\beta\left(E_{v}-E_{u}\right)}
$$

Eq. (1.23) tells us that the selected probability $P_{a d d}$ satisfies the detailed balance. 
As we can find from Ref. [45], around the critical temperature, the Wolff algorithm indeed is a much faster algorithm than the Metropolis algorithm. Since we want to improve the efficiency of our simulation, when we study the target system with other algorithms as for instance Glauber dynamics, the Wolff algorithm normally is used to thermalize the system to its equilibrium state around $T_{c}$ so that we can save a lot of time to deal with the measurement process.

\subsubsection{Kawasaki Dynamics}

Both Glauber dynamics and Wolff algorithm are invented for the Ising model in which the magnetization is allowed to fluctuate in time. However, there are some situations that require us to keep the magnetization to be a constant, for instance in the context of lattice gas models. We call the Ising model with constant density of magnetization the conserved-order-parameter (COP) Ising model. Kawasaki dynamics [50] is an algorithm for the COP Ising model.

Unlike what we have done for the normal Ising model with Glauber dynamics, in which we attempt to flip a single spin at one step, now two neighboring sites are selected in each time step. We try to exchange their spin values, and the change will be rejected or accepted through the Metropolis probability

$$
P(u \rightarrow v)= \begin{cases}e^{-\beta\left(E_{v}-E_{u}\right)} & \text { if } E_{v}-E_{u}>0 \\ 1 & \text { otherwise }\end{cases}
$$

We have proven that this probability fulfills detailed balance. It can be shown that it also satisfies ergodicity, within the set of states with the same magnetization. This algorithm turns out to be not so efficient. A faster choice is that instead of choosing two neighboring spins, we select two spins at random positions, and then do the exchange dynamics. This non-local exchange dynamics is faster than the normal Kawasaki dynamics, and obtains the same equilibrium results; it can therefore be used for thermalization.

For now, three well-used algorithms for Ising-like models are introduced. Certainly, there are more algorithms worth to be mentioned, we recommend Refs. [45, $51,52]$ for more details.

\subsection{Finite Size Scaling}

After collecting data from Monte Carlo simulations, it is always worth to ask what we can learn from them. For systems with a second-order phase transition, the behavior at the critical point attracts a lot of attention. Normally, we are interested in the behavior of quantities in the thermodynamic limit, where the system size goes to infinite. Unfortunately, due to the limited power of a computer, we can only simulate systems with relatively small sizes. A method is required that can extract critical exponents and the critical temperature from data obtained with small sizes, and finite-size scaling (FSS) is such a method. 
To understand how FSS works, we employ the magnetic susceptibility $\chi$ as an example, and it is defined as

$$
\chi(L)=\beta N\left(\left\langle m^{2}\right\rangle-\langle m\rangle^{2}\right)
$$

Based on the theory of FSS [45], at the critical point, the susceptibility is rescaled as

$$
\chi=L^{\gamma / \nu} \bar{\chi}\left(L^{1 / \nu} T^{\prime}\right)
$$

where $T^{\prime}=\left|T-T_{c}\right| / T_{c}$ is the reduced temperature.
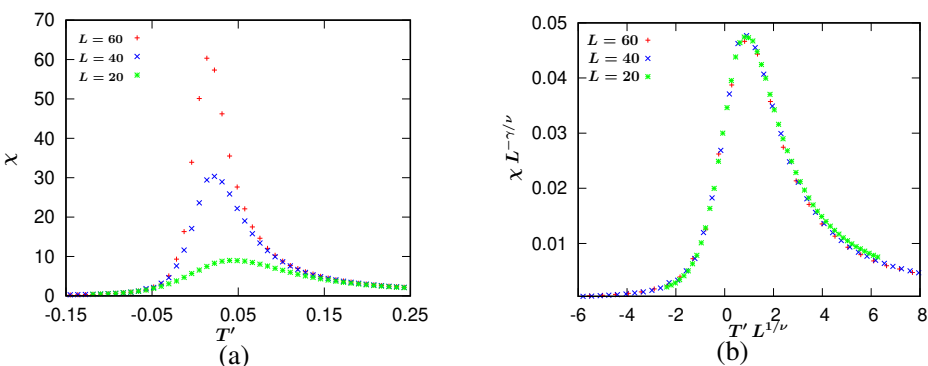

(b)

Figure 1.4: (a) The magnetic susceptibility as a function of temperature. (b) By scaling the susceptibility as $\chi=L^{\gamma / \nu} \bar{\chi}\left(L^{1 / \nu} T^{\prime}\right)$, where $T^{\prime}=\left|T-T_{c}\right| / T_{c}$ is the reduced temperature, it provides us the approximate values of the equilibrium exponents $\gamma \approx$ $1.76, \nu \approx 1.0$, and the critical temperature $T_{c} \approx 2.26$.

The scaling results shown in Figure 1.4 demonstrate the power of the FSS: when the susceptibility curves for different system sizes are scaled on top of each other, it provides us the proper values of the equilibrium exponents $\gamma$ and $\nu$ and the critical temperature $T_{c}$. The FSS is a powerful tool that helps us to determine accurately many critical properties.

\subsection{Thesis outline}

Until now we have provided a basic introduction into anomalous diffusion, and introduced some background of Ising-like models. Then the simulation methods are described in some detail, followed by three specific algorithms. We have also discussed finite size scaling, which is a useful method to analyze the data generated from Monte Carlo simulations.

In chapter 2, the anomalous diffusion is reported as a common phenomenon at the critical point for both $2 D$ and $3 D$ Ising models. We have numerically proven that the GLE is a proper model to describe the anomalous diffusion in the Ising model. Besides, we find that the anomalous exponent can be used to measure the dynamical exponent for Ising-like systems.

We then explore more of the anomalous diffusion near but not exactly at the critical point in chapter 3 . The diffusion exponent flows are measured for temperatures around 
$T_{c}$, and we obtain that for $T<T_{c}$, there is no true exponent, instead it has a peak value in the exponent flow diagram. While for $T>T_{c}$, the true exponent does exist. We find that the diffusion exponent is flowing away from the critical value of the true exponent from both sides, indicating that these results could be treated as a method to identify the phase transition in the Ising system.

In chapter 4 , we measure the dynamical exponent of the $2 D$ scalar $\phi^{4}$ model, which is a model claimed to belong to the same universality class as the $2 D$ Ising model, via two independent methods: directly measuring the decay of the correlation function $\langle m(t) m(0)\rangle$, then obtain $z$ from these data, and the scaling of the MSD $\left\langle[m(t)-m(0)]^{2}\right\rangle$. Our results confirm that the $2 D$ scalar $\phi^{4}$ model indeed shares the dynamical exponent with the $2 D$ Ising model, indicating that they belong to the same universality class.

After this chapter, the bond-diluted Ising model with different bond concentrations is considered in chapter 5, and we find that the dynamical exponent increases to infinity when the bond concentration approaches the percolation threshold, which is referred to as "super slowing down" behavior.

Up to this point, what we have investigated are mainly numerical results. In chapter 6 , we try to figure out the possibility to derive analytically the dynamical properties of a model in the set of Ising systems. The 2D Ising model with Kawasaki dynamics is studied. Its Fourier modes are treated as the dynamical eigenmodes, from which we calculate the MSD and autocorrelation function analytically in approximation. The results are consistent with findings in the previous chapters and the analytic methods help us eliminate the strong deviations arising from finite-size effects.

Finally, we conclude the thesis in chapter 7, and some unsolved problems are proposed for future studies. 

CHAPTER 2

\section{Generalized Langevin Equation Formulation for Anomalous Diffusion in the Ising Model at the Critical Temperature}

We consider the two- (2D) and three-dimensional (3D) Ising model on a square lattice at the critical temperature $T_{c}$, under Monte-Carlo spin flip dynamics. The bulk magnetization and the magnetization of a tagged line in the 2D Ising model, and the bulk magnetization and the magnetization of a tagged plane in the $3 \mathrm{D}$ Ising model exhibit anomalous diffusion. Specifically, their mean-square displacement increases as power-laws in time, collectively denoted as $\sim t^{c}$, where $c$ is the anomalous exponent. We argue that the anomalous diffusion in all these quantities for the Ising model stems from time-dependent restoring forces, decaying as power-laws in time - also with exponent $c$ - in striking similarity to anomalous diffusion in polymeric systems. Prompted by our previous work that has established a memory-kernel based Generalized Langevin Equation (GLE) formulation for polymeric systems, we show that a closely analogous GLE formulation holds for the Ising model as well. We obtain the memory kernels from spin-spin correlation functions, and the formulation allows us to consistently explain anomalous diffusion as well as anomalous response of the Ising model to an externally applied magnetic field in a consistent manner. 


\subsection{Introduction}

Despite the progress as mentioned in Sec.1.1, which model describes an instance of (experimentally) observed anomalous diffusion is often the subject of fierce debate, as evidenced by the recent case of anomalous diffusion observed for tracer particles in cell cytoplasm [18, 19, 20, 21, 22, 23], where all three of the above stochastic models have been fitted to the experimental data $[24,25,19,20,21,22,23,26]$. For physical systems where the dynamical rules for particle movement are known (in contrast to a complicated medium like a cell cytoplasm), one would expect to have a much easier task to model anomalous diffusion, yet it can still remain quite a challenge.

For polymeric systems, where anomalous diffusion is commonplace, it is only recently that Panja has established that the anomalous diffusion for tagged monomers are explained by "restoring forces" that decay as a power-law in time with the anomalous exponent of diffusion [53, 54]. From these characteristics it has been shown that anomalous diffusion in polymeric systems can be modeled by a Generalized Langevin Equation (GLE) with a memory kernel, and it belongs to the class of fBm [55].

The $\mathrm{fBm}$ characteristics of anomalous diffusion have been verified for flexible $[56,57]$ and semiflexible polymers [58], and polymer membranes [59, 60, 61]. Importantly, they have been used to successfully explain the dynamics of translocation of polymers across membranes [62, 63, 64, 65]. The fBm model framework has been generalized/extended to the linear transport regime for flexible polymers [54], and has similarly been used to explain field-driven polymer translocation [66] and polymer adsorption [67] for weak fields and adsorption energies. It has also found applications in strong nonlinear regimes for flexible polymers [68].

In this chapter, we take on characterizing anomalous diffusion in magnetization space for the Ising model on a square lattice at the critical temperature, undergoing Monte-Carlo spin-flip dynamics. That the total magnetization for this model exhibits anomalous diffusion has been reported by one of us in Ref. [28]. Additionally, we report that the magnetization of a tagged line in the 2D Ising model, and the magnetization of a tagged plane in the 3D Ising model, also exhibit anomalous diffusion. We argue that the anomalous diffusion for all these quantities for the Ising model stems from time-dependent restoring forces, decaying as power-laws in time - with the anomalous exponent of diffusion — in striking similarity to polymeric systems, and show that a closely analogous GLE formulation holds for the Ising model as well. We obtain the memory kernel from spin-spin correlation functions, and the formulation allows us to consistently explain anomalous diffusion as well as anomalous response of the Ising model to an externally applied magnetic field in a consistent manner.

The organization of this chapter is as follows. In Sec. 2.2 we introduce the Ising model and report the anomalous exponents of magnetization. In Sec. 2.3 we explain how restoring forces - that hold the key to anomalous diffusion - develop and work. In Sec. 2.4 we develop the GLE formulation for anomalous diffusion in the Ising model. The chapter is concluded with a discussion in Sec. 2.5. 
Generalized Langevin Equation Formulation for Anomalous Diffusion in the Ising Model at the Critical Temperature

\subsection{Anomalous diffusion in the Ising model at the crit- ical temperature}

\subsubsection{The model and dynamics}

We consider the Ising model on a square lattice. The Hamiltonian, at zero external magnetic field, is rewritten as

$$
\mathcal{H}_{0}=-J \sum_{\langle i j\rangle} s_{i} s_{j},
$$

where $s_{i}= \pm 1$ is the spin at site $i$, and $J$ is the coupling constant of interaction among the spins. The summation runs over all the nearest-neighbor spins. The linear size of the system is $L$; i.e., $0 \leq(i, j)<L$. Our samples satisfy periodic boundary conditions at all times, and all properties we report here are studied (or measured) at the critical temperature $T_{c}$.

The key quantity of focus in this chapter is the mean-square displacement (MSD) for magnetization $M(t)$ at time $t$ as

$$
\left\langle\Delta M^{2}(t)\right\rangle=\left\langle[M(t)-M(0)]^{2}\right\rangle,
$$

where $M(t)$ can take several forms. All angular brackets in this chapter, including those in Eq. (2.2), denote ensemble average. In the two-dimensional (2D) Ising model, we consider the respective cases where it is the bulk magnetization $M_{2 D, b}$, or the "line magnetization" $M_{2 D, l}$, the magnetization of a tagged line of spins in the $y$-direction. Similarly, in the three-dimensions, we consider the bulk magnetization $M_{3 D, b}$ and the magnetization $M_{3 D, p}$ of a tagged $x z$-plane.

We simulate the dynamics of the system using Monte Carlo moves, following the Metropolis algorithm. At any time-step a spin is randomly selected to flip, and the resulting change $\Delta E$, where $E$ is the energy of the system, is measured. The move is accepted with unit probability if $\Delta E \leq 0$; if not, then the move is accepted with the usual Metropolis probability $e^{-\Delta E /\left(k_{B} T_{c}\right)}$, where $k_{B}$ is the Boltzmann constant.

All simulation results reported here use $k_{B}=J=1$.

\subsubsection{Anomalous diffusion in the Ising model}

Let us denote by $D$ the spatial dimension of the support of the tagged magnetization given by $M$, meaning $D=1$ for a tagged line and $D=2$ for bulk in the $2 \mathrm{D}$ Ising model, while for the 3D Ising model $D=2$ for a tagged plane and $D=3$ for bulk. At short times $t \lesssim 1$, the individual spin flips in the model are uncorrelated, and since there are $L^{D}$ spins all together in these entities spatial dimensions,

$$
\left\langle\Delta M^{2}(t)\right\rangle \simeq L^{D} t
$$

At long times, $t \gg L^{z_{c}}$, where the $z_{c}$ is the dynamic exponent for the Ising model at $T_{c}$, we expect $\langle M(t) M(0)\rangle=0$. This means that

$$
\left\langle\Delta M^{2}(t)\right\rangle \equiv\left\langle[M(t)-M(0)]^{2}\right\rangle_{t \gg L^{z_{c}}}=2\left\langle M^{2}\right\rangle
$$


which is a purely equilibrium quantity which we can calculate from the equilibrium spin-spin correlations. We then have

$$
\left\langle M^{2}\right\rangle=\sum_{i \in L^{D}} \sum_{j \in L^{D}}\left\langle s_{i} s_{j}\right\rangle=\sum_{i \in L^{D}} \sum_{j \in L^{D}} r_{i j}^{2-d-\eta} \approx \int_{1}^{L} \frac{d^{D} r}{r^{d-2+\eta}} \sim L^{2 D-d+2-\eta},
$$

where $r_{i j}$ is the Euclidean distance between the two spins $i$ and $j, d$ is the spatial dimension of the model (i.e., $d=2$ and 3 for two- and three-dimensional Ising models respectively), and the critical exponent $\eta$ is related to $\gamma$ and $\nu$ via the scaling relation $2-\eta=\gamma / \nu$. (Note this result requires an integral $\int_{1}^{L} \frac{d^{D} r}{r^{d-2+\eta}}$ to be dominated by large $r$, which is why we have excluded line magnetization $D=1$ in three dimensions $d=3$ from this chapter.)

We now make the scaling assumption of an intervening power law with time

$$
\left\langle\Delta M^{2}(t)\right\rangle \propto t^{c}
$$

connecting across intermediate times from Eq. (2.3) at $t \simeq 1$ to Eqs. (2.4-2.5) at $t \simeq L^{z_{c}}$. The match at $t \simeq 1$ forces $\left\langle\Delta M^{2}(t)\right\rangle \simeq L^{D} t^{c}$, and the match at large time $L^{z_{c}}$ then requires $L^{D+c z_{c}} \simeq L^{2 D-d+\gamma / \nu}$, leading to

$$
c=\frac{D-d+\gamma / \nu}{z_{c}} .
$$

The full scaling prediction valid for all $t \gg 1$ is then

$$
\left\langle\Delta M^{2}(t)\right\rangle / L^{2 D-d+\gamma / \nu}=f\left(t / L^{z_{c}}\right)
$$

where $f(x) \simeq x^{c}$ for $x \ll 1$. Using the values of the critical exponents corresponding to $k_{B}=J=1$, as presented in Table 2.1 , the explicit power laws for $1 \lesssim t \lesssim L^{z_{c}}$ become

$$
\begin{gathered}
\left\langle\Delta M_{2 D, l}^{2}(t)\right\rangle \sim L t^{(\gamma / \nu-1) / z_{c}} \approx L t^{0.35} \\
\left\langle\Delta M_{2 D, b}^{2}(t)\right\rangle \sim L^{2} t^{\gamma /\left(\nu z_{c}\right)} \approx L^{2} t^{0.81} \\
\left\langle\Delta M_{3 D, p}^{2}(t)\right\rangle \sim L^{2} t^{(\gamma / \nu-1) / z_{c}} \approx L^{2} t^{0.48} . \\
\left\langle\Delta M_{3 D, b}^{2}(t)\right\rangle \sim L^{3} t^{\gamma /\left(\nu z_{c}\right)} \approx L^{3} t^{0.97},
\end{gathered}
$$

indicating that anomalous diffusion in the Ising model is ubiquitous at the critical temperature. As pointed out earlier, the results of the bulk magnetization were first obtained by one of us in Ref. [28].

The power-laws in Eq. (2.9) are verified in Figure 2.1. To obtain these data, we first thermalised the system. We then produced a number of independent time-series of $M(t)$, from which we measured $\left\langle\Delta M^{2}(t)\right\rangle$. In some of the plots in Figure 2.1 we notice a small deviation from the power-laws at late times: we have verified that this is caused by periodic boundary conditions - they are different when free boundary conditions are employed. Two examples of this can be found in Appendix A. 
Generalized Langevin Equation Formulation for Anomalous Diffusion in the Ising Model at the Critical Temperature
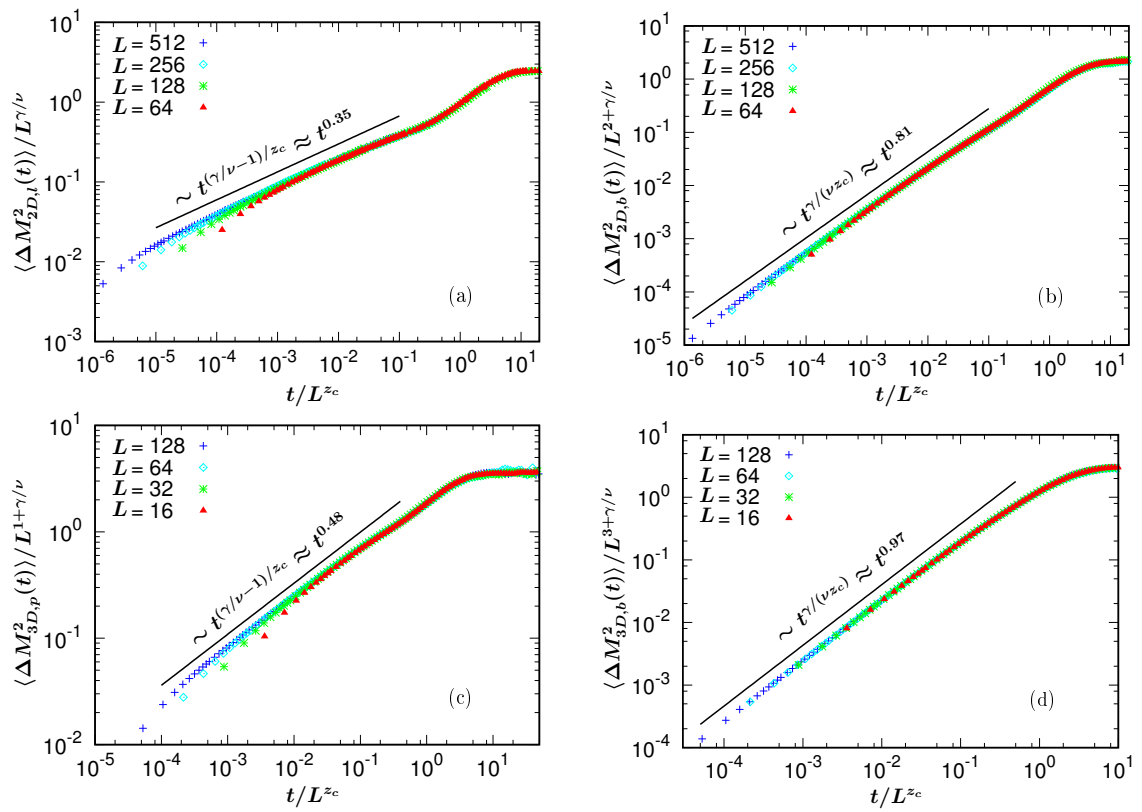

Figure 2.1: The mean-square displacement (MSD) of the magnetization $\left\langle\Delta M^{2}(t)\right\rangle$ in the Ising model at $T_{c}$ : (a) tagged line magnetization for the 2D Ising model, (b) bulk magnetization for the 2D Ising model, (c) tagged plane magnetization for the 3D Ising model, and (d) bulk magnetization for the 3D Ising model. The $x$ - and $y$-axes are scaled according to Eq. (2.8) leading to excellent data collapse over different $L$. The black solid lines denote the power-laws shown in Eq. (2.6). 


\begin{tabular}{c|c|c|c|c}
\hline \hline $\begin{array}{c}\text { Ising model } \\
\text { dimension } d\end{array}$ & $\gamma$ & $\nu$ & $z_{c}$ & $T_{c}$ \\
\hline \hline 2 & $7 / 4$ & 1 & $2.1665(12)$ & $\frac{2}{\ln (1+\sqrt{2})}$ \\
\hline 3 & $1.237075(10)$ & $0.629971(4)$ & $2.03(4)$ & $4.5116174(2)$ \\
\hline
\end{tabular}

Table 2.1: The relevant critical exponents and the critical temperature in the Ising model [29, 32, 69], using $k_{B}=J=1$ for the critical temperature $T_{c}$.

\subsection{Restoring forces: the physics of anomalous diffu- sion in the Ising model}

In this section we focus on the physics of anomalous diffusion. We argue that anomalous diffusion in the Ising model stems from restoring forces, in close parallel to polymeric systems.

\subsubsection{Restoring forces}

Imagine that the value of the tagged magnetization $M$ changes by an amount $\delta M$ due to thermal spin flips on the tagged line at $t=0$. Due to the interactions dictated by the Hamiltonian, the spins within and surrounding the tagged region, in the ensuing times, will react to this change. This reaction will be manifest in the two following ways: (a) the surrounding spins will to some extent adjust to the change over time, and (b) during this time the value of $M$ will also readjust to the persisting values of the surrounding spins, undoing at least a part of $\delta M$. It is the latter that we interpret as the result of "inertia" of the surrounding spins that resists changes in $M$, and the resistance itself acts as the restoring force to the changes in the tagged magnetization.

Since the imposed change $\delta M$ will be partially undone for $t>0$, we can expect the "velocity" autocorrelation function $\langle\dot{M}(0) \dot{M}(t)\rangle$ to be negative, an ingredient that we will use to establish the connection between the restoring forces and anomalous diffusion in Sec. 2.3.3.

\subsubsection{The time-decay behavior of restoring forces}

The main ingredient to connect the restoring forces and anomalous diffusion lies in how the former decays in time. To this end, we first consider the following thoughtexperiment, along the line described above in Sec. 2.3.1. On an equilibrated set of samples of the two-dimensional Ising model we create a small excess tagged magnetization $\delta M$ at $t=0$ with the constraint that we do not allow this excess to be subsequently undone; this corresponds to an imposed evolution of the tagged magnetization $d M(t) / d t=(\delta M) \delta(t)$, where $\delta(t)$ is the Kronecker delta function. The 
Generalized Langevin Equation Formulation for Anomalous Diffusion in the Ising Model at the Critical Temperature
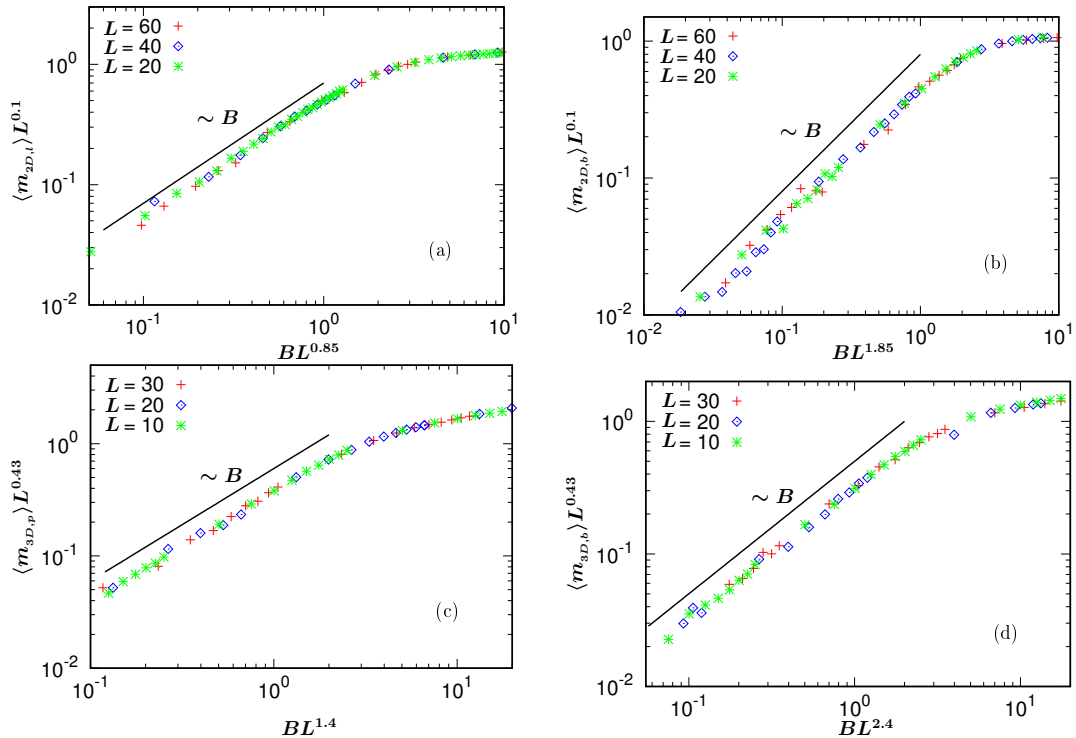

Figure 2.2: Plots showing the scaling form $\langle m\rangle L^{\kappa} \sim f\left(B L^{\lambda}\right)$ with $\kappa-\lambda=D-d+$ $\gamma / \nu$, confirming Eq. (2.11). The (numerically found) values of $\lambda$ are 0.1 in $2 \mathrm{D}$ and 0.43 in 3D: (a) $\left\langle m_{2 \mathrm{D}, l}\right\rangle$ (b) $\left\langle m_{2 \mathrm{D}, b}\right\rangle$, (c) $\left\langle m_{3 \mathrm{D}, p}\right\rangle$ and (d) $\left\langle m_{3 \mathrm{D}, b}\right\rangle$ (note: $\gamma / \nu \approx 1.75$ in $2 \mathrm{D}$ and $\approx 1.97$ in $3 \mathrm{D})$. 


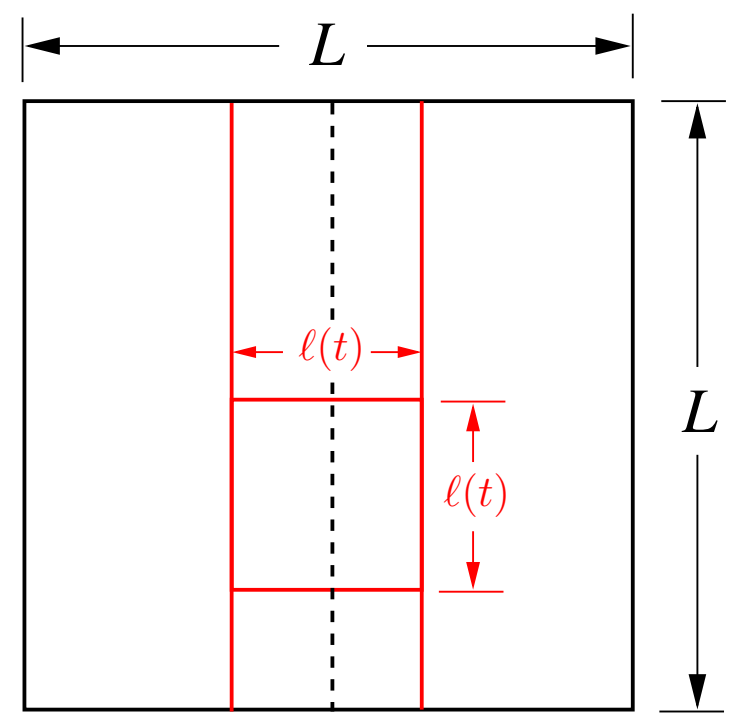

Figure 2.3: The thought experiment performed on the tagged line magnetization for the two-dimensional Ising model. A small excess (line) magnetization $\delta M_{2 \mathrm{D}, l}$ is created on the tagged line of spins, denoted by the dashed line, with the constraint that we do not allow the excess magnetization to be undone. Up to time $t$, this action creates a rectangular zone of width $\ell(t) \sim t^{1 / z_{c}}$ around the tagged line, shown by the red solid lines, which we can consider equilibrated to the new situation, in the following sense. If we consider the red square of size $\ell(t) \times \ell(t)$, then after time $t$ the spins therein will all have equilibrated to the segment of the tagged line within that square, and vice versa.

resulting restoring force at later time $t$ we will then write as

$$
f(t)=-k(t) \delta M
$$

where we interpret $k(t)$ as the magnetic analogue of a spring constant: in conventional magnetic language this is related to the susceptibility of the tagged magnetization through $k^{-1}=L^{D} \chi$.

For long times $t \gg L^{z_{c}}$ our spring constant will be the equilibrium one which is given by the equilibrium Fluctuation Theorem as

$$
k^{-1}=\beta\left\langle M^{2}\right\rangle \sim L^{2 D-d+\gamma / \nu} .
$$

Equation (2.11) can be confirmed by equilibrating samples under the magnetic analogue of an externally applied force, which is an external field applied to the tagged magnetization (i.e., the field is applied on the domain of support of the magnetization), such that the Hamiltonian becomes $\mathcal{H}=\mathcal{H}_{0}-M B$. We then expect a mean tagged magnetization density $\langle m\rangle=M L^{-D}=k^{-1} B L^{-D} \simeq B L^{D-d+\gamma / \nu}$ at small values 
Generalized Langevin Equation Formulation for Anomalous Diffusion in the Ising Model at the Critical Temperature

of $B$, which is the manifestation of linear response of the system under weak external forcing. More generally, we can expect a full scaling form $\langle m\rangle L^{\kappa} \sim f\left(B L^{\lambda}\right)$ for some $\kappa$ and $\lambda$, where the scaling function $f(x)$ has the property that $f(x \rightarrow \infty) \rightarrow$ constant, and $f(x \rightarrow 0) \sim x$ due to the linear dependence of $\langle m\rangle$ on $B$ as $B \rightarrow 0$. The latter condition implies that $\kappa-\lambda=D-d+\gamma / \nu$.

The scaling form $\langle m\rangle=M L^{-D}=k^{-1} B L^{-D} \simeq B L^{D-d+\gamma / \nu}$ with $\kappa-\lambda=$ $D-d+\gamma / \nu$ is confirmed in Figure 2.2. The quantity $\lambda$ is numerically found to be 0.1 and 0.43 for Ising models in two- and in three-dimensions respectively.

For intermediate times we expect equilibrium response to be achieved only locally across a length-scale $\ell(t) \sim t^{1 / z_{c}}$ within and around the tagged zone (see Figure 2.3). Within a region of the tagged zone of side $\ell(t)$ we then expect a contribution of tagged magnetization $\langle\Delta M\rangle_{\ell(t)} \sim B \ell(t)^{2 D-d+\gamma / \nu}$. Adding the response from $(L / \ell(t))^{D}$ such regions then leads to

$$
\langle M(t)\rangle=k(t)^{-1} B \sim B L^{D} \ell(t)^{D-d+\gamma / \nu} \sim B L^{D} t^{c},
$$

where the exponent $c$ is as already given in Eq. (2.7). The various cases of this result are verified in Figure 2.4.
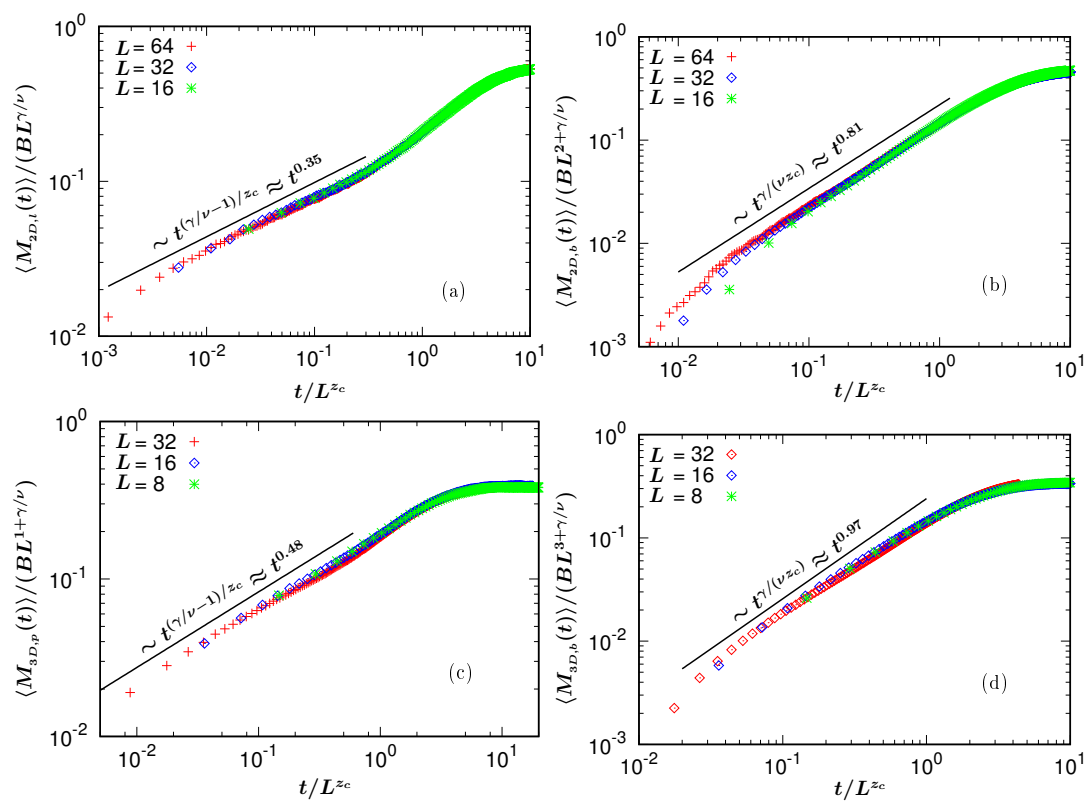

Figure 2.4: Average magnetization as a function of time when the magnetic field is switched on for the equilibrated samples at $t=0$ : (a) tagged line magnetization $\left\langle M_{2 D, l}(t)\right\rangle$ and (b) bulk magnetization $\left\langle M_{2 D, b}(t)\right\rangle$ for the 2D Ising model, and (c) the tagged plane magnetization $\left\langle M_{3 D, p}(t)\right\rangle$ and (d) bulk magnetization $\left\langle M_{3 D, b}(t)\right\rangle$ for the 3D Ising model.

To summaries, the key result of this section is that if we create an excess tagged 
magnetization $\delta M$ at $t=0$ and do not allow it to relax away, then a time-dependent restoring force $f(t)$ acts such as would reverse it, where

Tagged line magnetization in two-dimensions:

$$
f_{\text {rest }}(t)=-L^{-1} t^{\left.-(\gamma / \nu-1) / z_{c}\right)} \delta M_{2 \mathrm{D}, l},
$$

Bulk magnetization in two-dimensions:

$$
f_{\text {rest }}(t)=-L^{-2} t^{-\gamma /\left(\nu z_{c}\right)} \delta M_{2 \mathrm{D}, b},
$$

Tagged plane magnetization in three-dimensions:

$$
f_{\text {rest }}(t)=-L^{-2} t^{-(\gamma / \nu-1) / z_{c}} \delta M_{3 \mathrm{D}, p},
$$

and Bulk magnetization in three-dimensions:

$$
f_{\text {rest }}(t)=-L^{-3} t^{-\gamma /\left(\nu z_{c}\right)} \delta M_{3 \mathrm{D}, b} \text {. }
$$

\subsubsection{Anomalous diffusion stems from these restoring forces}

The main result of Sec. 2.3.2, for which $\dot{M}(t) \propto \delta(t)$, can be represented as the following formal time-dependent "impedance-admittance relation" [62, 63, 64]

$$
f_{\text {rest }}(t)=-\int_{0}^{t} d t^{\prime} \mu\left(t-t^{\prime}\right) \dot{M}\left(t^{\prime}\right),
$$

with a causal memory function given by $\mu(t)=k(t) \sim L^{-D} t^{-c}$ as in Eq. (2.13) for $t>0$, and $\mu(t)=0$ for $t<0$. Equation (2.14) is obtained from Eq. (2.13) using the superposition principle: the total restoring force at time $t$ is a sum of all preceding $\delta M$ values weighted by the (power-law) memory kernel $\mu$. In this formulation, $\dot{M}(t)$ plays the role of current through a circuit, with $f_{\text {rest }}(t)$ playing the role of the voltage, and $\mu(t)$ is the time-dependent impedance. On the one hand, this formulation means that $\left\langle f_{\text {rest }}(t) f_{\text {rest }}\left(t^{\prime}\right)\right\rangle_{\dot{M}=0}=\mu\left(\left|t-t^{\prime}\right|\right)$, while on the other, we can invert Eq. (2.14) to express $\dot{M}(t)$ as a function of $f_{\text {rest }}(t)$ involving the time-dependent admittance $a(t)$ as

$$
\dot{M}(t)=-\int_{0}^{t} d t^{\prime} a\left(t-t^{\prime}\right) f_{\text {rest }}\left(t^{\prime}\right)
$$

and correspondingly $\left\langle\dot{M}(t) \dot{M}\left(t^{\prime}\right)\right\rangle_{f_{\text {rest=0 }}}=a\left(t-t^{\prime}\right)$, with the impedance and the admittance following the relation $\tilde{a}(s) \tilde{\mu}(s)=1$ in the Laplace space $s$. These imply that $a(t)=\langle\dot{M}(t) \dot{M}(0)\rangle_{f_{\text {rest }=0}} \sim-L^{D} t^{c-2}$. Integrating this quantity twice in time using the Green-Kubo relation we obtain

$$
\langle\Delta M(t)\rangle^{2} \simeq L^{D} t^{c}
$$

(we will return to this calculation more formally in Sec. 2.4), leading us not only to the anomalous exponents of Eq. (2.6), but also the correct $L$-dependent prefactors for the data collapse in Figure 2.1. The results are summarized in Table 2.2. 
Generalized Langevin Equation Formulation for Anomalous Diffusion in the Ising Model at the Critical Temperature

\begin{tabular}{c|c|c}
\hline \hline Magnetization of & $\mu(t)$ & $\langle\Delta M(t)\rangle^{2}$ \\
\hline \hline tagged line in 2D & $L^{-1} t^{-(\gamma / \nu-1) / z_{c}}$ & $L t^{(\gamma / \nu-1) / z_{c}}$ \\
\hline bulk in 2D & $L^{-2} t^{-\gamma /\left(\nu z_{c}\right)}$ & $L^{2} t^{\gamma /\left(\nu z_{c}\right)}$ \\
\hline tagged plane in 3D & $L^{-2} t^{-(\gamma / \nu-1) / z_{c}}$ & $L^{2} t^{(\gamma / \nu-1) / z_{c}}$ \\
\hline bulk in 3D & $L^{-3} t^{-\gamma /\left(\nu z_{c}\right)}$ & $L^{3} t^{\gamma /\left(\nu z_{c}\right)}$ \\
\hline
\end{tabular}

Table 2.2: Memory functions and anomalous diffusion of magnetization in the Ising model. The anomalous diffusion applies only until the terminal relaxation time scaling $\sim L^{z_{c}}$.

\subsubsection{Restoring forces and anomalous diffusion: a similar story for polymer dynamics}

Although slightly off-topic, we now briefly point out that the dynamics of the restoring forces and anomalous diffusion for magnetization in the Ising model is practically identical to those in polymer dynamics [53, 54, 56, 62, 63, 64, 66, 67]. This subsection forms the basis of Sec. 2.4, where we discuss the Generalized Langevin Equation formulation of anomalous diffusion in the Ising model.

Even though anomalous diffusion in polymeric systems is the norm rather than an anomaly, we specifically pick the Rouse polymer to demonstrate the similarity; for instance, the anomalous diffusion of a tagged monomer in the Rouse model, which scales as $t^{2 \nu /(1+2 \nu)}$ until the terminal Rouse time $\tau_{R} \sim N^{1+2 \nu}$ (and diffusively thereafter). Here, $\nu$ is the Flory exponent ( $=3 / 4$ in two and $\approx 0.588$ in three dimensions), and $N$ is the polymer length.

Imagine that we move a tagged monomer by a small distance $\delta \vec{r}$ at $t=0$ and hold it at its new position $\forall t>0$ (just like in our thought experiment of Sec. 2.3.2, where we created an excess magnetization $\delta M$ at $t=0$ and did not allow it to be undone). For more details, we refer the reader to Ref. [56], where we analyzed this thought experiment. In the ensuing time, all the monomers within a backbone distance $n_{t} \sim t^{1 /(1+2 \nu)}$, counting away from the tagged monomer will equilibrate to the new position of the tagged monomer. However, the end-to-end distance of this equilibrated set of monomers is no longer their natural spatial extent $\left(\sim n_{t}^{\nu}\right)$, but is instead stretched by an amount $\propto \delta \vec{r}$. With the (entropic) spring constant of these $n_{t}$ equilibrated monomers scaling as $\sim n_{t}^{-2 \nu}$, the mean force the tagged monomer experiences at its new position is then given by $\vec{f}_{\text {rest }}(t) \sim-n_{t}^{-2 \nu}(\delta \vec{r}) \sim-t^{-2 \nu /(1+2 \nu)}(\delta \vec{r})$ [i.e., force $=($ spring constant $) \times$ stretching distance $]$. This relation is identical in formulation to Eqs. (2.13), and the rest of the emulated analysis (2.14-2.16) leads one to the result that the mean-square displacement of the tagged monomer increases as $t^{2 \nu /(1+2 \nu)}$. Of course this result only holds till the polymer's terminal Rouse time $\tau_{R} \sim N^{1+2 \nu}$, just like the anomalous diffusion in the Ising model survives until the terminal relaxation time scaling $\sim L^{z_{c}}$.

The reader may find a comparison of Table I in Ref. [54] and Table 2.2 of this chapter interesting. Note that at the critical temperature the system size $L$ corresponds to the polymer length $N$ : both systems reach criticality when these parameters reach 
infinity.

\subsection{Generalised Langevin Equation formulation for anomalous diffusion in the Ising model}

In the previous section we focused on the physics of the anomalous diffusion in the Ising model. Using a thought experiment we argued that the time-decay behavior of the restoring forces is the key ingredient to describe the relation between the restoring forces and anomalous diffusion in terms of the memory function $\mu(t)$. Equation (2.14) and its inverse formulation led us not only to the anomalous exponents for the meansquare displacements, but also to the correct $L$-dependent prefactors to obtain the data collapse in Figure 2.1. These results pose now an interesting question: could we formulate a stochastic differential equation for the anomalous diffusion in the Ising model?

A comparison to the corresponding relations between the restoring forces and anomalous diffusion for polymeric systems - taken up in the elaborate paper [54] by one of us - offers a clue to a possible answer to the above question. Therein the (anomalous) dynamics of a tagged monomer is shown to be described by the two following stochastic differential equations involving the monomeric velocity $v(t)$, the respective internal and external forces $f(t)$ and $f_{\text {ext }}$ that it experiences, and the memory function $\mu(t)$ :

$$
\begin{aligned}
\gamma v(t) & =f(t)+q_{1}(t) \\
f(t) & =-\int_{0}^{t} d t^{\prime} \mu\left(t-t^{\prime}\right) v\left(t^{\prime}\right)+f_{\mathrm{ext}}+q_{2}(t) .
\end{aligned}
$$

Here $\gamma$ is the viscous drag on the monomer by the surrounding (effective) medium, $q_{1}(t)$ and $q_{2}(t)$ are two noise terms satisfying $\left\langle q_{1}(t)\right\rangle=\left\langle q_{2}(t)\right\rangle=0$, and the fluctuationdissipation theorems (FDTs) $\left\langle q_{1}(t) q_{1}\left(t^{\prime}\right)\right\rangle \propto \gamma \delta\left(t-t^{\prime}\right)$ and $\left\langle q_{2}(t) q_{2}\left(t^{\prime}\right)\right\rangle \propto \mu\left(t-t^{\prime}\right)$ respectively. (Note that factors of $k_{\mathrm{B}} T$ terms have been suppressed from these equations.) The idea behind Eq. (2.17) is that while the internal restoring force builds on the history of the monomeric velocity, the latter simply responds instantaneously to the force it experiences.

Similarity between the second one of Eq. (2.17) and Eq. (2.14) prompts us to propose the total force as

$$
f(t)=-\int_{0}^{t} d t^{\prime} \mu\left(t-t^{\prime}\right) \dot{M}\left(t^{\prime}\right)+f_{\mathrm{ext}}+g(t),
$$

for the Ising model, where $f_{\text {ext }}$ is simply the externally applied force, such as a magnetic field. The noise term $g(t)$ satisfies the condition that $\langle g(t)\rangle=0$ and the corresponding FDT $\left\langle g(t) g\left(t^{\prime}\right)\right\rangle=\mu\left(\left|t-t^{\prime}\right|\right)$. As we have done before, Eq. (2.18) can be inverted, in terms of the admittance $a(t)$, to write

$$
\dot{M}(t)=-\int_{0}^{t} d t^{\prime} a\left(t-t^{\prime}\right) f\left(t^{\prime}\right)+\omega(t) .
$$


Generalized Langevin Equation Formulation for Anomalous Diffusion in the Ising Model at the Critical Temperature

The noise term $\omega(t)$ similarly satisfies $\langle\omega(t)\rangle=0$, and the FDT $\left\langle\omega(t) \omega\left(t^{\prime}\right)\right\rangle=a(\mid t-$ $\left.t^{\prime} \mid\right)$. The impedance and the admittance are related to each other in the Laplace space as $\tilde{a}(s) \tilde{\mu}(s)=1$.

Additionally, we propose that in the Monte-Carlo dynamics, magnetization in the Ising model instantaneously responds to the internal force as

$$
\zeta \dot{M}=f(t)+q(t),
$$

with a damping coefficient $\zeta$ and a corresponding white noise term $q(t)$. Thereafter, having combined Eqs. (2.18) and (2.20) we obtain

$$
\zeta \dot{M}=-\int_{0}^{t} d t^{\prime} \mu\left(t-t^{\prime}\right) \dot{M}\left(t^{\prime}\right)+f_{\mathrm{ext}}+g(t)+q(t),
$$

or

$$
\dot{M}=\int_{0}^{t} d t^{\prime} \theta\left(t-t^{\prime}\right)\left[f_{\mathrm{ext}}+g\left(t^{\prime}\right)+q\left(t^{\prime}\right)\right],
$$

where in the Laplace space $\tilde{\theta}(s)[\zeta+\tilde{\mu}(s)]=1$.Here, without the $\zeta$ term $\theta(t)$ is identical to $a(t)$, introduced in Eq. (2.15).

At zero external magnetic field the dynamics of $M$ simplifies to

$$
\dot{M}=\int_{0}^{t} d t^{\prime} \theta\left(t-t^{\prime}\right)\left[g\left(t^{\prime}\right)+q\left(t^{\prime}\right)\right],
$$

similar to Eq. (2.17) for polymeric systems. Without further ado, we then simply follow Ref. [54] to conclude, with $\mu(t) \sim L^{-D} t^{-c}$, that

$$
\left\langle\dot{M}(t) \dot{M}\left(t^{\prime}\right)\right\rangle=-\theta\left(t-t^{\prime}\right) \sim-L^{D}\left(t-t^{\prime}\right)^{c-2} .
$$

Note that in Eq. (2.24) we have ignored the $\zeta$ term, which essentially means that we are ignoring the (uninteresting) time-scale $\lesssim \zeta^{-1}$. Subsequently, by integrating the Eq. (2.24) twice in time using the Green-Kubo relation, the MSD of the magnetization can be obtained as

$$
\left\langle\Delta M^{2}(t)\right\rangle \sim L^{D} t^{c}
$$

which are the same results obtained in Eq. (2.16). An example verification for the velocity autocorrelation function (2.24) can be found in Appendix B.

This GLE formulation demonstrates that the anomalous diffusion in the Ising model at the critical temperature is non-Markovian, with a power-law memory function $\mu(t)$. Quite simply, if $\mu(t) \sim t^{-c}$, then the anomalous diffusion exponent is $c$.

\subsubsection{Numerical confirmation of the GLE formulation (and deter- mination of the damping coefficient $\zeta$ )}

It is now imperative that we numerically test our proposed GLE formulation for anomalous diffusion for the Ising model. Our key test is to check the FDT 
$\left\langle f_{\text {rest }}(t) f_{\text {rest }}\left(t^{\prime}\right)\right\rangle_{\dot{M}=0}=\mu\left(t-t^{\prime}\right)$, for which we describe our approach below, followed by presentation of the numerical results.

Conceptually, the task is simple. At a fixed value of $M$, i.e., $\dot{M}=0$ at all times, we need to numerically measure $\left\langle f_{\text {rest }}(t) f_{\text {rest }}\left(t^{\prime}\right)\right\rangle$. However, we cannot measure forces in the Monte Carlo dynamics of the model since by definition one does not have forces in discrete lattice models. In order to circumvent this difficulty, we use Eq. (2.20) as a proxy for $f(t)$ by choosing $\zeta=1$ and use the value $\overline{\dot{M}}_{\text {free }}$ (see below), which would have applied to the tagged magnetization if the fixed $M$ constraint were to be lifted at that time.

We start with a thermalised system at $t=0$. For $t>0$ we fix the value of $M$ (this does not mean that all tagged spins are frozen), which we achieve by performing non-local spin-exchange moves. Specifically, for the magnetization of a tagged line in $2 \mathrm{D}$ and tagged plane in $3 \mathrm{D}$, we avoid extreme values of $M$ by choosing to fix it in the interval $-0.2<m=M L^{-D}<0.2$ (note that in the scaling limit all values of $m$ belong to this range). We then keep taking snapshots of the system at regular intervals, and compute, at every snapshot (denoted by $t$ ), the expectation value $\dot{\dot{M}}_{\text {free }}(t)$ conditional on the current configuration, which for our Metropolis MonteCarlo dynamics is given by,

$$
\overline{\dot{M}}_{\text {free }}(t)=\sum_{i \in \text { tagged }}\left(-2 s_{i}\right) \operatorname{Min}\left(1, e^{-\Delta E_{i} /\left(k_{\mathrm{B}} T_{c}\right)}\right)=f(t) .
$$

This means that for every snapshot we take, we consider an attempt to flip each spin in turn and find the expected change in $M$ which would have occurred if this move had been implemented, totaled over all the spins.

Finally, we note that since simulations are performed for finite systems with $M$ fixed at its $t=0$ value, in any particular run we need a non-zero value of $f_{\text {ext }}=$ $-\langle f(t)\rangle$ acting to sustain the initial value of $M$. Further, given that that in our proxy measurement for $f(t)$ using Eq. (2.20) we can only access $f_{\text {rest }}(t)+f_{\text {ext }}$, but not $f_{\text {rest }}(t)$ directly, it is the quantity

$$
\begin{aligned}
\Gamma\left(\dot{M}_{2 \mathrm{D}, l}(t)\right) & =\left\langle\dot{M}(t) \dot{M}\left(t^{\prime}\right)\right\rangle-\langle\dot{M}(t)\rangle\left\langle\dot{M}\left(t^{\prime}\right)\right\rangle \\
& =L^{2 D}\left(\left\langle f(t) f\left(t^{\prime}\right)\right\rangle-\langle f(t)\rangle\left\langle f\left(t^{\prime}\right)\right\rangle\right)
\end{aligned}
$$

that should correctly proxy $\left\langle g(t) g\left(t^{\prime}\right)\right\rangle_{\dot{M}=0}=\mu\left(t-t^{\prime}\right)$, and we expect the following results:

$$
\begin{gathered}
\Gamma\left(\dot{M}_{2 \mathrm{D}, l}(t)\right) \sim L t^{-(\gamma / \nu-1) / z_{c}} \approx L t^{-0.35}, \\
\Gamma\left(\dot{M}_{2 \mathrm{D}, b}(t)\right) \sim L^{2} t^{-\gamma /\left(\nu z_{c}\right)} \approx L^{2} t^{-0.81}, \\
\Gamma\left(\dot{M}_{3 \mathrm{D}, p}(t)\right) \sim L^{2} t^{-(\gamma / \nu-1) / z_{c}} \approx L^{2} t^{-0.48}, \\
\Gamma\left(\dot{M}_{3 \mathrm{D}, b}(t)\right) \sim L^{3} t^{-\gamma /\left(\nu z_{c}\right)} \approx L^{3} t^{-0.97} .
\end{gathered}
$$

These results are verified in Figure 2.5, along with the effective exponents as numerically obtained derivative $d(\ln \Gamma) / d(\ln t)$ as insets. 
Generalized Langevin Equation Formulation for Anomalous Diffusion in the Ising Model at the Critical Temperature

In Figure 2.5, the data quality for 3D bulk at long times suffers from the difficulty of collecting statistically independent datasets at long times. There are also small deviations from the power-laws at late times for line magnetization in $2 \mathrm{D}$ and plane magnetization in $3 \mathrm{D}$; we suspect that these relate to similar deviations observed in Figure 2.1.
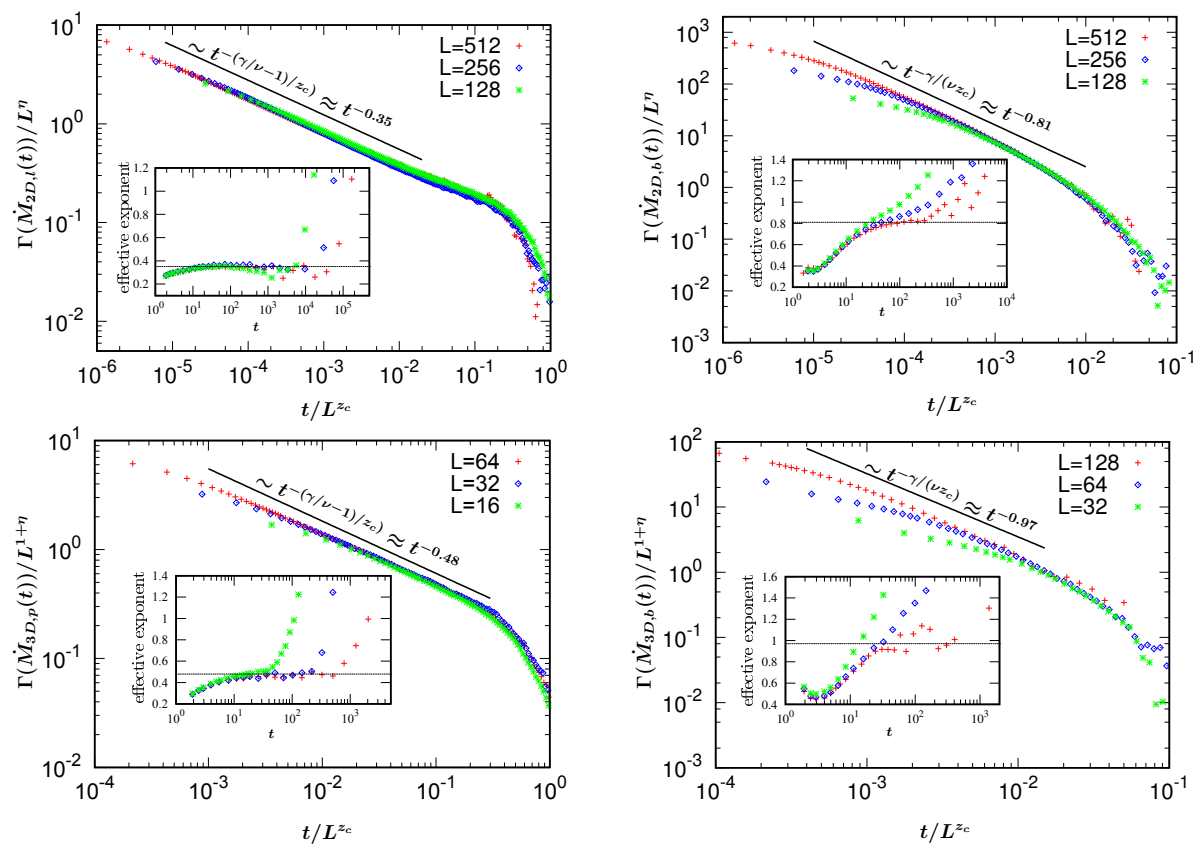

Figure 2.5: Plots showing the scaling of (a) $\Gamma\left(\dot{M}_{2 \mathrm{D}, l}(t)\right)$, (b) $\Gamma\left(\dot{M}_{2 D, b}(t)\right)$, (c) $\Gamma\left(\dot{M}_{3 D, p}(t)\right)$ and (d) $\Gamma\left(\dot{M}_{3 D, b}(t)\right)$ as a function of $t / L^{z_{c}}$. Insets show the effective exponent, numerically obtained derivative $-d(\ln \Gamma) / d(\ln t)$, with the dotted lines denoting the expected values of the slope. Note that the critical exponent $\eta$ is related to $\gamma$ and $\nu$ via the scaling relation $\eta=2-\gamma / \nu$.

Additionally, we have followed the procedure described in Ref. [70] to obtain the power-law exponents from data in Figure 2.5; these values, together with the error bars, for the respective largest system sizes, can be found in Table 2.3. We have chosen the largest system sizes for this purpose since they contain the least amount of finite size effects.

\subsubsection{The GLE formulation for driven Ising systems}

The GLE formulation (2.19-2.20) also describes the anomalous response of the model to external magnetic fields. Starting from Eq. (2.22) and focusing on the response to an external field $f_{\text {ext }}=B$ switched on at $t=0$, we readily obtain the results of Eq. (2.12) for the tagged magnetization induced for times $1 \lesssim t \lesssim L^{z_{c}}$ by taking an ensemble 


\begin{tabular}{c|c|c}
\hline \hline System & $\begin{array}{c}\text { Estimated exponent from } \\
\text { Figure 2.5 }\end{array}$ & Expected value \\
\hline \hline tagged line in 2D, & $-0.35 \pm 0.02$ & -0.35 \\
$L=512$ & $-0.81 \pm 0.03$ & -0.81 \\
\hline bulk in 2D, $L=512$ & $-0.47 \pm 0.02$ & -0.48 \\
\hline $\begin{array}{c}\text { tagged plane in 3D, } \\
L=64\end{array}$ & $-0.97 \pm 0.04$ & -0.97 \\
\hline bulk in 3D, $L=128$ & & \\
\hline
\end{tabular}

Table 2.3: Power-law exponents from data in Figure 2.5, together with error bars, for the respective largest system sizes. Evidently, the data compare well with the expected exponents.

average that reduces the noise terms $g(t)$ and $q(t)$ to zero; specifically,

$$
\begin{gathered}
\left\langle M_{2 \mathrm{D}, l}(t)\right\rangle \sim B L t^{(\gamma / \nu-1) / z_{c}} \approx B L t^{0.35}, \\
\left\langle M_{2 \mathrm{D}, b}(t)\right\rangle \sim B L^{2} t^{\gamma /\left(\nu z_{c}\right)} \approx B L^{2} t^{0.81}, \\
\left\langle M_{3 \mathrm{D}, p}(t)\right\rangle \sim B L^{2} t^{(\gamma / \nu-1) / z_{c}} \approx B L^{2} t^{0.48}, \\
\left\langle M_{3 \mathrm{D}, b}(t)\right\rangle \sim B L^{3} t^{\gamma /\left(\nu z_{c}\right)} \approx B L^{3} t^{0.97},
\end{gathered}
$$

which have been verified already in Figure 2.4.

\subsection{Discussion}

In summary, in this chapter we report that the Ising model in two and three dimensions exhibits ubiquitous anomalous diffusion behavior at the critical temperature. We have performed four case studies for this: the bulk magnetization, magnetization of a tagged line in $2 \mathrm{D}$ and that of a tagged plane in 3D. We have argued that the anomalous diffusion stems from a time-dependent restoring force that involves a power-law memory kernel. We have derived these power-laws as well as the corresponding $L$-dependent prefactors.

Further, we have shown that the physics of anomalous diffusion in the Ising model bears strong similarities to that in polymeric systems, allowing us to propose a GLE description for anomalous diffusion in the Ising model. We have also verified that the anomalous diffusion for the tagged magnetization in the Ising model belongs to the fractional Brownian motion ( $\mathrm{fBm}$ ) class, although we do not explicitly report it in this chapter. We have numerically tested the specific aspects of the GLE (such as the FDTs), and the GLE description is also consistent with the observed anomalous response of magnetization to externally applied magnetic fields. In a future paper, work on which is already in progress, we will expand the GLE formulation to the Ising model around the critical temperature.

Having said the above, we have not mathematically proved the GLE, neither the $\mathrm{fBm}$, for the Ising model. Some other kinds of models may also be consistent with the 
Generalized Langevin Equation Formulation for Anomalous Diffusion in the Ising Model at the Critical Temperature

anomalous diffusion behavior observed by us in this chapter. They should, however, feature restoring forces, transient response to an external magnetic field, and a negative velocity autocorrelation function (observed in Figure 2.7), in a consistent manner as presented here. In particular, we note that the Ising model we study here is at equilibrium at $T_{c}$, and therefore time-reversible, so anomalous diffusion models that are developed for time-irreversible aging-type systems will not be applicable here.

Finally, we believe that the anomalous diffusion of the order parameter at the critical temperature can be found in other Ising-like systems, and if so, the GLE formulation introduced in this chapter can be employed to describe those anomalous behavior as well. In particular, if we know the critical temperature $T_{c}$, the critical exponents $\gamma$ and $\nu$ for a specific Ising-like system, then this method can be used to obtain the critical dynamical exponent $z_{c}$ from the power-laws as well as the scaling of the terminal time $\sim L^{z_{c}}$ (in other words, anomalous diffusion can be effectively used to measure the critical dynamic exponent $z_{c}$ ). We will test these ideas in our future work.

\subsection{Appendixes}

\subsubsection{Appendix A: Boundary conditions effect.}
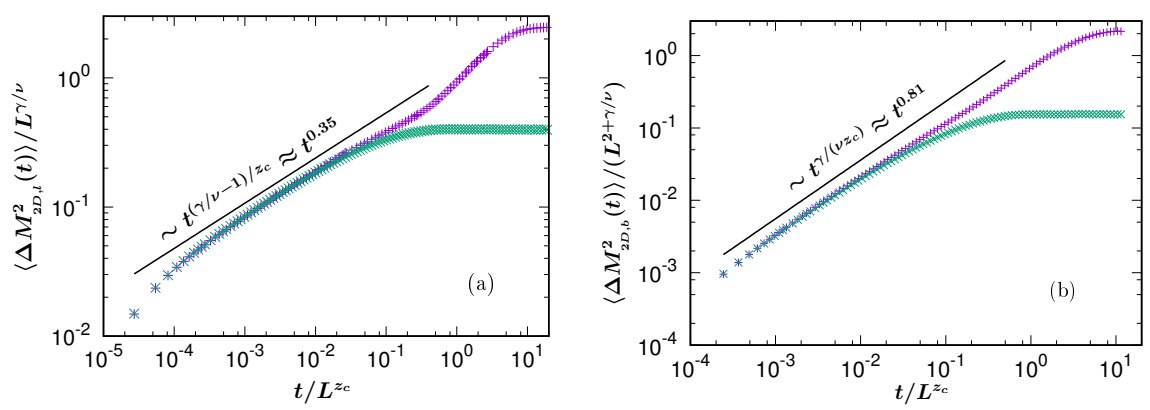

Figure 2.6: Comparison of the mean-square displacement for the line [Figure (a), system size $L=128$ ], and bulk [Figure (b), system size $L=64$ ] magnetization for the Ising model in 2D, with periodic boundary conditions (magenta pluses) and free boundary conditions (green crosses). The data for the two different boundary conditions are on top of each other in the scaling regime, differing only at late times.

In this appendix we demonstrate, in Figure 2.6, using two examples that the deviations from the power-law behavior at late times, as seen in Figure 2.1 are indeed caused by the periodic boundaries.

\subsubsection{Appendix B: Velocity autocorrelation of the magnetizations}

In this appendix, in Figure 2.7 we present a verification for the Green-Kubo relation used to convert the velocity autocorrelation function (2.24) to anomalous diffusion 


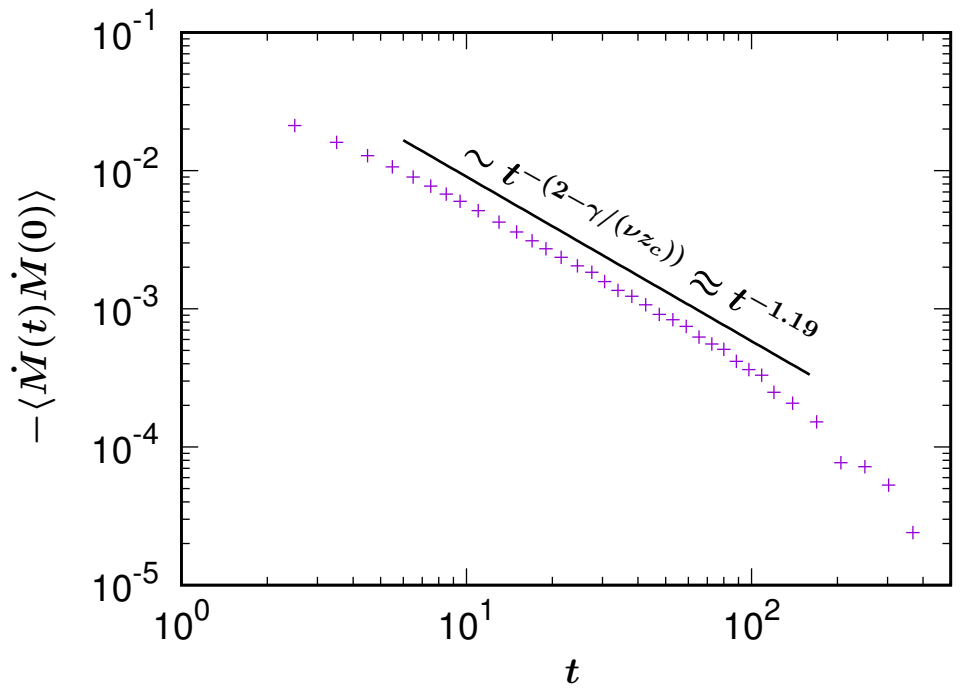

Figure 2.7: Velocity autocorrelation function $\langle\dot{M}(t) \dot{M}(0)\rangle$ of the 2D bulk magnetization as a function of $t$. This quantity is negative, and behaves $\sim-t^{-2-\gamma /(\nu z)} \approx$ $-t^{-1.19}$. The system size used in the simulation is $L=30$.

(2.25): i.e., for an anomalous diffusion exponent $c$ the velocity autocorrelation function anomalous exponent must be $c-2$, as well as having an overall negative sign in front. 
CHAPTER 3

\section{Anomalous diffusion in the off-critical Ising model with Monte Carlo spin-flip dynamics}

We study the equilibrium dynamics of the bulk magnetization $M$ for the two- and three-dimensional Ising model with Monte Carlo spin-flip dynamics near, but not exactly at, the critical temperature $T_{c}$. We report that the data for the mean-square deviation (MSD) of the bulk magnetization $\left\langle\Delta M^{2}(t)\right\rangle$ collapse on two separate mastercurves for $T>T_{c}$ and for $T<T_{c}$. The mastercurves are of the form $\left\langle\Delta M^{2}(t)\right\rangle \mid T-$ $\left.T_{c}\right|^{\gamma} L^{-d} \sim \mathcal{F}\left(t\left|T-T_{c}\right|^{\nu z_{c}}\right)$, where $d$ is the spatial dimension of the system, $\mathcal{F}$ is a scaling function, $\gamma$ and $\nu$ are two critical exponents, and $z_{c}$ is the critical dynamical exponent. In the limit $x \rightarrow 0, \mathcal{F}(x) \sim x^{\gamma /\left(\nu z_{c}\right)}$ for both mastercurves. For large $x$, $\mathcal{F}(x) \sim x$ before saturation for $T>T_{c}$, and $\mathcal{F}(x) \rightarrow$ const. for $T<T_{c}$. 


\subsection{Introduction}

The term anomalous diffusion is used to describe a particle's mean square displacement deviating from its expected (or normal) behavior, viz. its linear increase in time. Although the term 'anomalous' has been coined to denote an anomaly, in the last decades it has been observed in a wide variety of systems [1], such as fractal systems and disordered media [2, 3, 71], financial markets [4], transport in (crowded) cellular interiors [5], migration of cells [6], bacteria [7], animal foraging [8], polymeric systems [53, 54, 55, 56, 62, 68] and biomembranes [72, 73].

Recently, we have shown that anomalous diffusion (of various forms of magnetization) is also a regular occurrence for Ising-like models at $T_{c}$, such as the Ising model with Monte Carlo spin-flip dynamics [28, 74] and Kawasaki dynamics [75], and the $\phi^{4}$ model [76]. For all these models we have developed a memory-based fractional Brownian motion ( $\mathrm{fBm}$ ) description of anomalous diffusion.

In this chapter, we supplement our work on Ising-like systems by studying the dynamics of magnetization at temperatures $T$ in the neighborhood of $T_{c}$. At $T \rightarrow T_{c}$, the anomalous exponent approaches the expected value of the exponent at $T_{c}$, i.e., $\gamma /\left(\nu z_{c}\right)$, consistent with Refs. [28, 74]. Specifically, we report that the data for the mean-square deviation (MSD) of the bulk magnetization $\left\langle\Delta M^{2}(t)\right\rangle$ for the two- (2D) and three-dimensional (3D) Ising model, simulated with Monte Carlo spin-flip dynamics, collapse on two separate mastercurves for $T>T_{c}$ and $T<T_{c}$. The mastercurves are of the form $\left\langle\Delta M^{2}(t)\right\rangle\left|T-T_{c}\right|^{\gamma} L^{-d} \sim \mathcal{F}\left(t\left|T-T_{c}\right|^{\nu z_{c}}\right)$, where $d$ is the spatial dimension of the system, $\mathcal{F}$ is a scaling function, $\gamma$ and $\nu$ are two critical exponents, and $z_{c}$ is the critical dynamical exponent (see Table 1.1). In the limit $x \rightarrow 0$, $\mathcal{F}(x) \sim x^{\gamma /\left(\nu z_{c}\right)}$ for both mastercurves, while for large $x, \mathcal{F}(x) \sim x$ before saturation for $T>T_{c}$, and $\mathcal{F}(x) \rightarrow$ const. for $T<T_{c}$.

The chapter is organized as follows. In Sec. 3.2 we introduce the Ising model and the Monte Carlo spin-flip dynamics. In Sec. 3.3 we present our data for the MSD of the bulk magnetization. The chapter is concluded in Sec. 3.4.

\subsection{The model and Monte Carlo spin-flip dynamics}

We consider the Ising model on square and cubic lattices with periodic boundary conditions. The Hamiltonian is given by

$$
\mathcal{H}=-J \sum_{\langle i j\rangle} s_{i} s_{j},
$$

where $s_{i}= \pm 1$ is the spin at site $i$, and $J$ is the coupling constant of interaction among the spins, and the summation runs over all pairs of neighboring sites. The linear size of the system is the number of spins; i.e., $0 \leq(i, j)<L$. The summation runs over all the nearest neighbor spins and $M=\sum_{i} s_{i}$ is the bulk magnetization.

We simulate the dynamics of the system using Monte Carlo moves, following the Metropolis algorithm. At any time-step a spin is randomly selected to flip, and the 
Anomalous diffusion in the off-critical Ising model with Monte Carlo spin-flip dynamics

resulting change $\Delta E$, where $E$ is the energy of the system, is measured. The move is accepted with unit probability if $\Delta E \leq 0$; if not, then the move is accepted with the usual Metropolis probability $e^{-E /\left(k_{B} \bar{T}\right)}$, where $k_{B}$ is the Boltzmann constant and $T$ the temperature.

All simulation results reported here use $k_{B}=J=1$. The temperature ranges are chosen to be $\left(T_{c}-0.1\right)<T<\left(T_{c}+0.1\right)$ and $T \neq T_{c}$, where $T_{c}=2 / \ln (1+\sqrt{2})$ and $4.5116174(2)$ [32] for the $2 D$ and $3 D$ Ising model respectively.

\subsection{Mean-square displacement of the bulk magnetiza- tion}

The mean-square displacement of the bulk magnetization is expressed as

$$
\left\langle\Delta M^{2}(t)\right\rangle=\left\langle[M(t)-M(0)]^{2}\right\rangle=2\left\langle M(t)^{2}\right\rangle-2\langle M(t) M(0)\rangle .
$$

The relaxation time of the system, $\tau$, scales with $T-T_{c}$ as

$$
\tau \sim\left|T-T_{c}\right|^{-\nu z_{c}}
$$

beyond which the correlation term $\langle M(t) M(0)\rangle$ must go to zero, leading to

$$
\left\langle\Delta M^{2}(t)\right\rangle \underset{t \gg \tau}{\longrightarrow} 2\left\langle M(t)^{2}\right\rangle=2 \frac{L^{d} \chi}{\beta} .
$$

Here $\chi$ is the magnetic susceptibility per spin. Close to the critical temperature it diverges as

$$
\chi \sim\left|T-T_{c}\right|^{-\gamma}
$$

where $\gamma$ and $\nu$ are two equilibrium exponents.

Together, Eqs. (3.2-3.5) suggest the scaling form for the MSD of the bulk magnetization

$$
\left\langle\Delta M^{2}(t)\right\rangle\left|T-T_{c}\right|^{\gamma} L^{-d} \sim \mathcal{F}\left(t\left|T-T_{c}\right|^{\nu z_{c}}\right),
$$

where the scaling function $\mathcal{F}(x) \sim x^{c}$ for $x \ll 1$, and $c$ is the anomalous diffusion exponent.

The scaling result (3.6) is verified in Figure 3.1. To obtain the corresponding data, the system is first thermalised using the Wolff algorithm. We then produce a number of independent time series of $M(t)$, from which we measure $\left\langle\Delta M^{2}(t)\right\rangle$.

\subsubsection{The effective exponent and its exponent flow diagram}

In order to further investigate the mean-square displacement of the bulk magnetization, we define its time-dependent effective exponent as

$$
c(t) \equiv \frac{\partial \ln \left\langle\Delta M^{2}(t)\right\rangle}{\partial \ln t},
$$



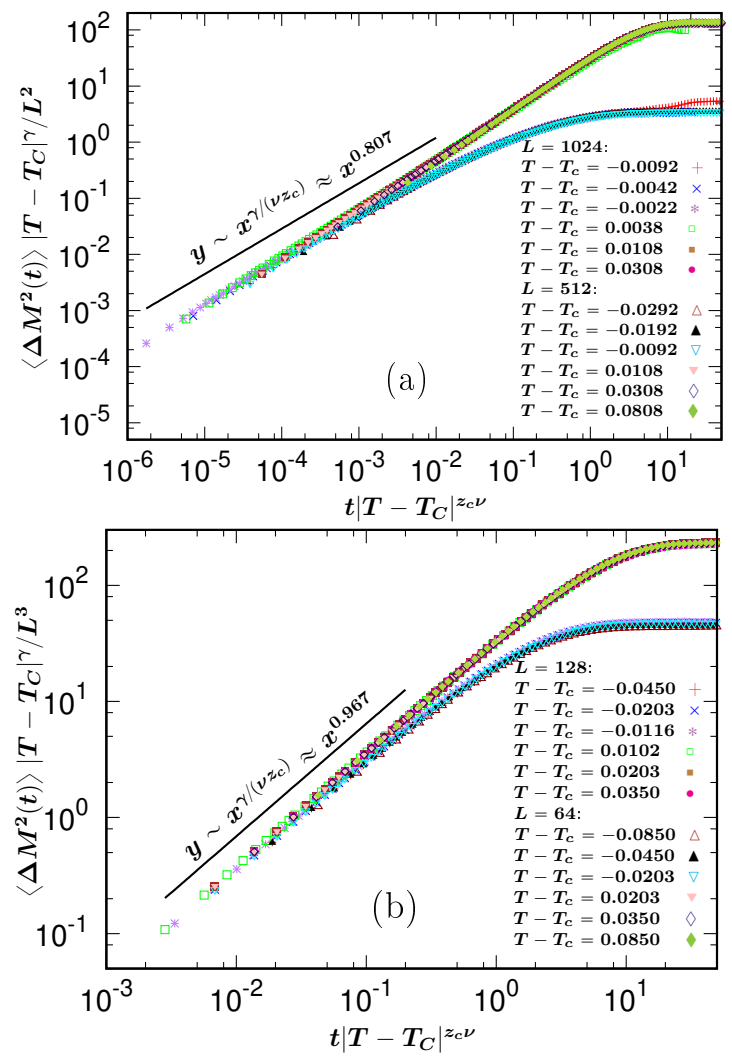

Figure 3.1: The scaling behavior of the MSD of the bulk magnetization $\left\langle\Delta M^{2}(t)\right\rangle$ in the (a) $2 D$ and (b) $3 D$ Ising model with Monte Carlo spin flip. The temperature ranges are chosen to be $T_{c}-0.1<T<T_{c}+0.1$ ( $T=T_{c}$ excluded). We have chosen $L=512$ and 1024 for the $2 D$, and $L=64$ and 128 for the $3 D$ systems. The data collapse to mastercurves; one each for $T>T_{c}$ (upper data) and $T<T_{c}$ (lower data). In the limit $x \rightarrow 0, \mathcal{F}(x) \sim x^{\gamma /\left(\nu z_{c}\right)}$ for both mastercurves. For large $x, \mathcal{F}(x) \sim x$ before saturation for $T>T_{c}$, and $\mathcal{F}(x) \rightarrow$ const. for $T<T_{c}$. 
Anomalous diffusion in the off-critical Ising model with Monte Carlo spin-flip dynamics
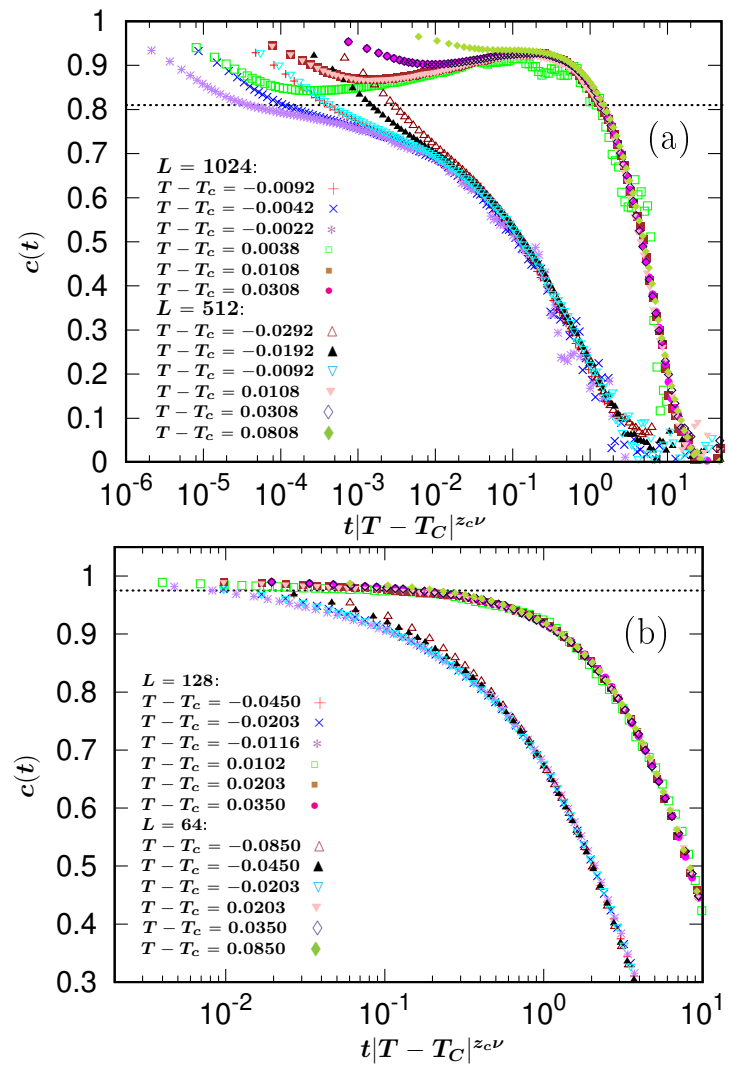

Figure 3.2: The effective exponent $c(t)$, as numerically obtained logarithmic derivative $\partial\left(\ln \left\langle\Delta M^{2}(t)\right\rangle\right) / \partial(\ln t)$. The dotted line denotes the expected value at $T=T_{c}$. The temperature ranges are chosen to be $T_{c}-0.1<T<T_{c}+0.1$, and $T \neq T_{c}$. (a) The results for 2D Ising model with $L=512$ and 1024, where the dotted line denotes the value $\gamma /\left(\nu z_{c}\right) \approx 0.807$; (b) The results for 3D Ising model with $L=64$ and 128 , where the value is $\gamma /\left(\nu z_{c}\right) \approx 0.967$ for the dotted line. Both the $2 \mathrm{D}$ and $3 \mathrm{D}$ results demonstrate that when $T \rightarrow T_{c}$, the slope of the MSD of the bulk magnetization approaches the critical value from above and below respectively for $T>T_{c}$ (upper data) and $T<T_{c}$ (lower data). 
which we compute numerically.

The data for the effective exponent can be found in Figure 3.2. Using the same $x$-axis variable in Figure 3.2 as that of Figure 3.1, we see that after scaling the data with Eq. (3.6), the curves for different temperatures again fall on to two mastercurves, respectively for below and for above $T_{c}$. Both mastercurves again demonstrate that in the limit $x \rightarrow 0, \mathcal{F}(x) \sim x^{\gamma /\left(\nu z_{c}\right)}$ for both mastercurves. For large $x, \mathcal{F}(x) \sim x$ before saturation for $T>T_{c}$, and $\mathcal{F}(x) \rightarrow$ const. for $T<T_{c}$.

Further, we also numerically compute the logarithmic derivative $\dot{c}(t) \equiv \partial c(t) / \partial \ln (t)$. The idea behind this is that if we can conceptually express $\dot{c}(t)$ as a function of $c(t)$; i.e., as an "exponent flow diagram" $\dot{c}=\mathcal{G}(c)$, then a true exponent will correspond to $\mathcal{G}(c)=0$.

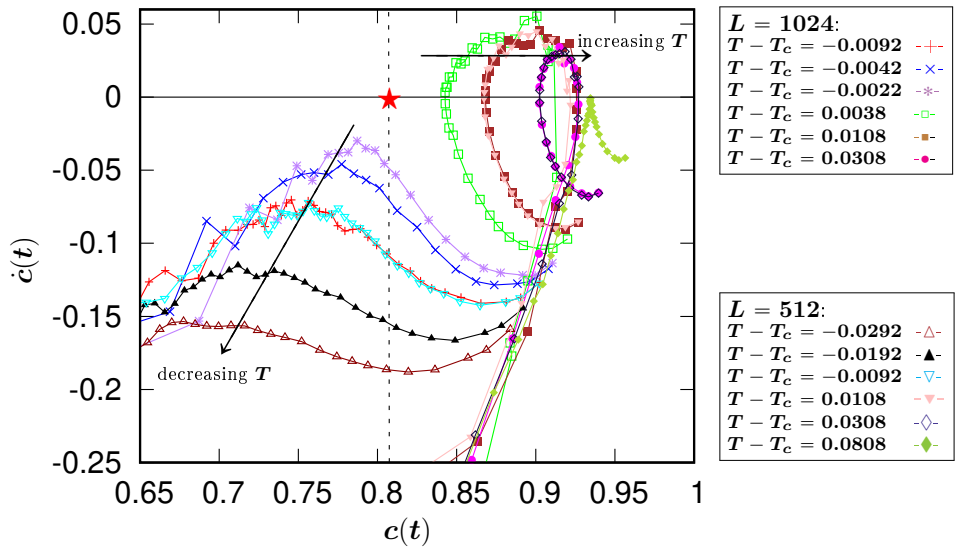

Figure 3.3: The exponent flow diagram for the MSD of the bulk magnetization for the 2D Ising model. Here, the system sizes are $L=512$ and 1024, and the temperature varies from $T_{c}-0.1$ to $T_{c}+0.1$. The true exponent at the critical temperature, $\approx 0.807$, appears at the intersection of the dotted and the dashed lines as a red star. Above and below $T_{c}$ the data flow away from this value in two different directions.

We plot the exponent flow diagram in Figure 3.3, with the true exponent $\approx 0.807$ denoted by a red star. (we refrain from the exponent flow diagram for the 3D Ising model since the numerical value of the exponent is very close to unity). In the figure we see that above and below $T_{c}$ the data flow away from this value in two different directions.

\subsection{Conclusion}

To conclude, we have studied the MSD of the bulk magnetization, $\left\langle\Delta M^{2}(t)\right\rangle$, for the 2D and 3D Ising model with Monte Carlo spin-exchange dynamics around the critical temperature $T_{c}$. We find that the data of the MSD of the magnetization around $T_{c}$ can be scaled as $\left\langle\Delta M^{2}(t)\right\rangle\left|T-T_{c}\right|^{\gamma} L^{-d} \sim \mathcal{F}\left(t\left|T-T_{c}\right|^{\nu z_{c}}\right)$ with a scaling function 
Anomalous diffusion in the off-critical Ising model with Monte Carlo spin-flip dynamics

$\mathcal{F}$, where $\gamma, \nu$ are two critical exponents, $d$ is the spatial dimension and $z_{c}$ is the critical dynamical exponent. For different temperatures the data collapse onto two mastercurves, one for below and the other for above $T_{c}$. In the limit $x \rightarrow 0, \mathcal{F}(x) \sim$ $x^{\gamma /\left(\nu z_{c}\right)}$ for both mastercurves. For large $x, \mathcal{F}(x) \sim x$ before saturation for $T>T_{c}$, and $\mathcal{F}(x) \rightarrow$ const. for $T<T_{c}$. These results are consistent with the anomalous exponent $\gamma /\left(\nu z_{c}\right)$, as reported in Refs. $[28,74]$ at $T_{c}$. 

CHAPTER 4

\section{Critical Dynamical Exponent of the Two-Dimensional Scalar $\phi^{4}$-Model with Local Moves}

We study the scalar one-component two-dimensional (2D) $\phi^{4}$-model by computer simulations, with local Metropolis moves. The equilibrium exponents of this model are well-established, e.g. for the $2 \mathrm{D} \phi^{4}$-model $\gamma=1.75$ and $\nu=1$. The model has also been conjectured to belong to the Ising universality class. However, the value of the critical dynamical exponent $z_{c}$ is not settled. In this chapter, we obtain $z_{c}$ for the 2D $\phi^{4}$-model using two independent methods: (a) by calculating the relative terminal exponential decay time $\tau$ for the correlation function $\langle\Phi(t) \Phi(0)\rangle$, and thereafter fitting the data as $\tau \sim L^{z_{c}}$, where $L$ is the system size, and (b) by measuring the anomalous diffusion exponent for the order parameter, viz., the mean-square displacement (MSD) $\left\langle\Delta \Phi^{2}(t)\right\rangle \sim t^{c}$ as $c=\gamma /\left(\nu z_{c}\right)$, and from the numerically obtained value $c \approx 0.80$, we calculate $z_{c}$. For different values of the coupling constant $\lambda$, we report that $z_{c}=2.17 \pm 0.03$ and $z_{c}=2.19 \pm 0.03$ for the two methods respectively. Our results indicate that $z_{c}$ is independent of $\lambda$, and is likely identical to that for the 2D Ising model. Additionally, we demonstrate that the Generalized Langevin Equation (GLE) formulation with a memory kernel, identical to those applicable for the Ising model and polymeric systems, consistently captures the observed anomalous diffusion behavior. 


\subsection{Introduction}

Despite the advances in the equilibrium properties of the $\phi^{4}$ model that were mentioned in Sec. 1.3.2, its critical dynamical properties are not settled.

As for the critical dynamical exponent, Blöte and Nightingale [77] have analyzed three variations of Ising-type models with next-nearest-neighbor interactions, and found that they share the same critical exponents, not only $\gamma$ and $\nu$, but also the critical dynamical exponent $z_{c}$. Further works have supported their results both in 2D and 3D $[78,79,80]$. For the 2D Ising model $z_{c}$ has been determined quite precisely as $z_{c}=2.1665 \pm 0.0012$ [29]. For the critical dynamical exponent of the 2D $\phi^{4}$ model, $z \approx 2$ was mentioned in Ref. [81], and the $\epsilon$-expansion method has shown that $z_{c} \in(2.04,2.14)$ [82]. Further, $z_{c}$ has been measured using the heat bath algorithm, yielding $z_{c}=1.9 \pm 0.21$ [83]. In short, the value of the critical dynamical exponent for the $\phi^{4}$-model still remains to be determined with higher precision.

In this chapter, we study the one-component 2D scalar $\phi^{4}$-model by computer simulations, i.e., Eq. (1.6), with local Metropolis moves. In order to settle the value of $z_{c}$, we employ two independent methods: (a) we calculate the relative terminal exponential decay time $\tau$ for the correlation function $\langle\Phi(t) \Phi(0)\rangle$, and thereafter fit the data as $\tau \sim L^{z_{c}}$, where $L$ is the system size, (b) we measure the mean-square displacement (MSD) of the order parameter $\left\langle\Delta \Phi^{2}(t)\right\rangle \sim t^{c}$ with $c=\gamma /\left(\nu z_{c}\right)$, and from the numerically obtained value $c \approx 0.80$ we calculate $z_{c}$. We report that $z_{c}=$ $2.17 \pm 0.03$ and $z_{c}=2.19 \pm 0.03$ for the two methods respectively. Our results suggest that $z_{c}$ is independent of $\lambda$, and is likely identical to that for the 2D Ising model.

Further, the numerical result $\left\langle\Delta \Phi^{2}(t)\right\rangle \sim t^{0.80}$ at the critical point means that $\Phi(t)$ undergoes anomalous diffusion. We argue that the physics of anomalous diffusion in the $\phi^{4}$-model at critical point is the same as for polymeric systems and the Ising model $[53,54,55,74]$, and therefore a GLE formulation that holds for the Ising model at criticality and for polymeric systems must also hold for the $\phi^{4}$-model. We obtain the force autocorrelation function for the $\phi^{4}$-model at $\dot{\Phi}=0$, and the results allow us to demonstrate the consistency between anomalous diffusion and its GLE formulation.

The chapter is organized as follows. In Sec. 4.2 we introduce the $\phi^{4}$-model and the dynamics, and then show the results of the correlation term $\langle\Phi(t) \Phi(0)\rangle$ and the mean-square displacement of the order parameter; from both we measure the critical dynamical exponent. In Sec. 4.3 we briefly explain how the restoring force works, which naturally leads us to the Generalized Langevin Equation (GLE) formulation for the anomalous diffusion in the $\phi^{4}$-model, and verify the GLE formulation for anomalous diffusion. The chapter is concluded in Sec. 4.4. 


\subsection{The Measurement of the Critical Dynamical Expo- nent}

\subsubsection{The Model and the Dynamics}

We consider the scalar one-component two-dimensional $\phi^{4}$-model on an $L \times L$ square lattice with periodic boundary conditions. The action is introduced in Eq. (1.6), and in this chapter we focus on $\lambda \leq 1$.

We simulate the dynamics of the system, i.e. Eq. (1.7), using Monte Carlo moves, with the Metropolis algorithm: we randomly select a site $i$, for which we try to change the existing value $\phi_{i}$ to a new value $\phi_{i}^{\prime}$, given by

$$
\phi_{i}^{\prime}=\phi_{i}+\Delta \phi\left(r-\frac{1}{2}\right)
$$

where $r$ is a random number uniformly distributed within [0,1), and following Refs. $[40,41]$, we set $\Delta \phi=3$. The resulting change of the action $\Delta \mathcal{S}$ after every attempted change in $\phi_{i}$ is calculated. The move is accepted if $\Delta \mathcal{S} \leq 0$; if not, then the move is accepted with the usual Metropolis probability $e^{-\Delta \mathcal{S}}$. With $\Delta \phi=3$, the acceptance rates are between $40 \%$ and $60 \%$.

In this chapter, all simulations have been performed on a $3.40 \mathrm{GHz}$ desktop PC running Linux. We mainly focus on three different values of $\lambda$, i.e. $\lambda=0.1,0.5,1.0$. The corresponding critical coupling constant $\beta_{c}$, obtained in Refs. [41, 84], are listed in table 4.1 .

\begin{tabular}{c|c}
\hline \hline$\lambda$ & Value of $\beta_{c}$ \\
\hline \hline 0.1 & $0.60647915(35)$ \\
\hline 0.5 & $0.686938(10)$ \\
\hline 1.0 & $0.680601(11)$ \\
\hline
\end{tabular}

Table 4.1: The value of $\beta_{c}$ for $\lambda=0.1,0.5$ and $1.0[41,84]$.

Next, we use two independent methods to measure the dynamical exponent $z_{c}$.

\subsubsection{Measurement of the Correlation function $\langle\Phi(t) \Phi(0)\rangle$}

In the first method, we measure the correlation function $\langle\Phi(t) \Phi(0)\rangle$ of the order parameter. To obtain the corresponding data, we run our simulations for $5 \times 10^{7}$ Monte Carlo steps per lattice site to thermalise the system. Subsequently, we keep taking snapshots of the system at regular intervals over a total time of $5 \times 10^{8}$ Monte Carlo steps per lattice site, and compute the order parameter $\Phi$ at every snapshot. From this data set we calculate $\langle\Phi(t) \Phi(0)\rangle$.

We use system sizes $L=30,40, \ldots, 90$ for each value of $\lambda$. The required CPU time is about 45 minutes for $L=30$, reaching about 6 hours for $L=90$. 
At long times we expect $\langle\Phi(t) \Phi(0)\rangle$ to behave as $\langle\Phi(t) \Phi(0)\rangle /\langle\Phi(0) \Phi(0)\rangle \sim$ $\exp (-t / \tau)$, and define $Q(t)=-\ln [\langle\Phi(t) \Phi(0)\rangle /\langle\Phi(0) \Phi(0)\rangle]$, leading us to expect

$$
Q(t) \sim t / \tau .
$$

We then calculate the relative value of terminal decay time $\tau$ by collapsing the $Q(t)$ data to a reference for every value of $\lambda$. More explicitly, for every value of $\lambda$ we choose the $Q(t)$ data for $L=30$ as reference, set its $\tau$-value to unity, and then collapse the rest of the $Q(t)$ for other values of $L$ to that reference, which yields us the relative value of $\tau$ for that value of $\lambda$. As an example, Figure 4.1(a) demonstrates this procedure: with a properly chosen relative value of $\tau$, the $\langle\Phi(t) \Phi(0)\rangle$ data for different system sizes collapse to the data of $L=30$.

At the critical temperature $\tau \sim \xi^{z_{c}}$, where $\xi$ is the correlation length. According to finite-size scaling theory, for finite system sizes $\xi$ needs to be replaced by $L$, i.e.,

$$
\tau \sim L^{z_{c}} .
$$

\begin{tabular}{c|c}
\hline \hline$\lambda$ & $z_{c}$ \\
\hline \hline 0.1 & $2.17 \pm 0.03$ \\
\hline 0.5 & $2.15 \pm 0.03$ \\
\hline 1.0 & $2.20 \pm 0.03$ \\
\hline
\end{tabular}

Table 4.2: The measured values of $z_{c}$ for the $2 \mathrm{D} \phi^{4}$-model at different $\lambda$. The critical dynamical exponent $z_{c}$ is calculated by fitting the data of the relative value of $\tau$ as $\tau \sim L^{z_{c}}$. The results indicate that the value of $z_{c}$ is likely independent of $\lambda$, which allows us to produce a single estimate of $z_{c}$, viz., $z_{c}=2.17 \pm 0.03$ (see main text).

The critical dynamical exponent $z_{c}$ is calculated by fitting the data of the relative value of $\tau$ with Eq. (4.3). Results of this procedure are shown in Figure 4.1(b). The corresponding values of $z_{c}$ can be found in Table. 4.2. The error bars in Table 4.2 are obtained from the best fits of Figure 4.1(b). These results indicate that the value of $z_{c}$ is likely independent of $\lambda$. If we do assume that, then we can combine the different numerical values for different $\lambda$ to produce a single estimate of $z_{c}$, viz., $z_{c}=2.17 \pm$ 0.03 .

\subsubsection{Mean-Square Displacement of the Order Parameter}

In the second method, we focus on the measurement of the mean-square displacement of the order parameter at time $t$, given by

$$
\left\langle\Delta \Phi^{2}(t)\right\rangle=\left\langle[\Phi(t)-\Phi(0)]^{2}\right\rangle .
$$

To obtain the data of the MSD of the order parameter, we first thermalise the system with $2 \times 10^{8}$ Monte Carlo moves per lattice site, then measure $\left\langle\Delta \Phi^{2}(t)\right\rangle$ in a further 
Critical Dynamical Exponent of the Two-Dimensional Scalar $\phi^{4}$-Model with Local Moves

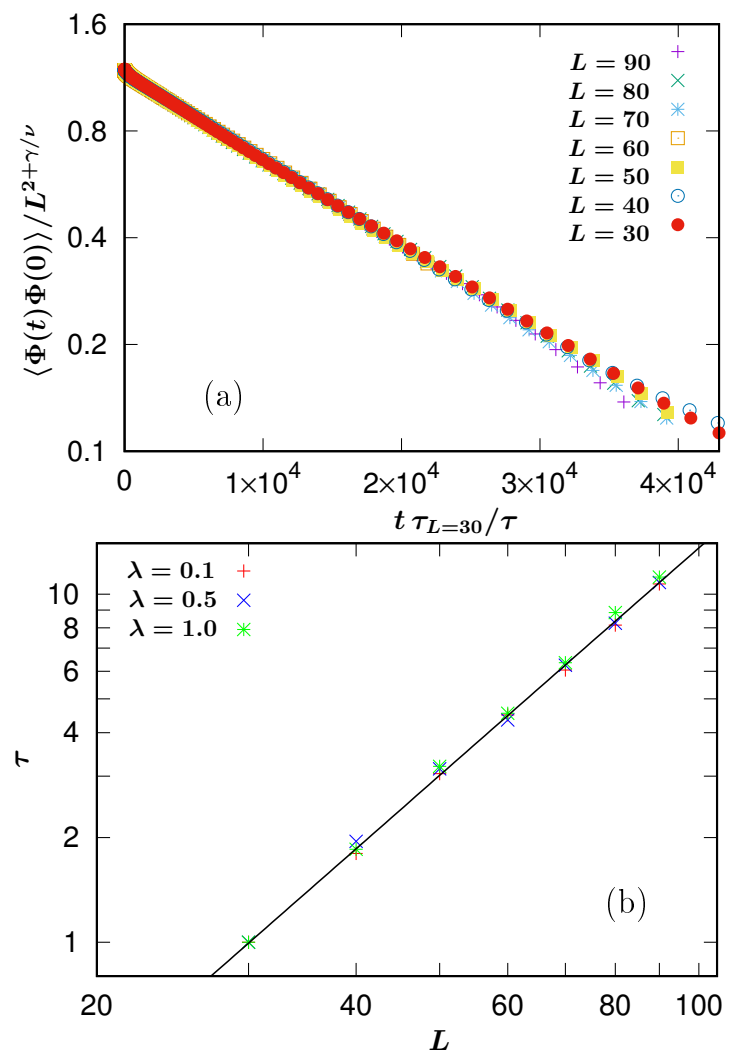

Figure 4.1: Measurement of $z_{c}$ from the $Q(t)$ data for different values of $\lambda$ at the critical point. (a) An example of the measurement process to obtain the relative value of terminal decay time $\tau$. In this figure $\lambda=0.1$ and $\tau_{L=30}$ is the terminal decay time of reference system size $L=30$. The data collapse (to the reference) is obtained by adjusting the relative values of terminal decay time for other system sizes; (b) Measurement of $z_{c}$ by fitting the data of the relative value of decay time $\tau$ as $\tau \sim$ $L^{z_{c}}$.The symbols represent the simulation results of $\tau$ and the solid line corresponds to $\tau \sim L^{2.17}$. 


\begin{tabular}{c|c}
\hline \hline$\lambda$ & $z_{c}$ \\
\hline \hline 0.1 & $2.20 \pm 0.03$ \\
\hline 0.5 & $2.18 \pm 0.02$ \\
\hline 1.0 & $2.20 \pm 0.04$ \\
\hline
\end{tabular}

Table 4.3: The critical dynamical exponent $z_{c}$, which is obtained from the numerically obtained $c$ with $c=\gamma /\left(\nu z_{c}\right)$, for the 2D $\phi^{4}$-model at different $\lambda$. The results, too, indicate that the value of $z_{c}$ is likely independent of $\lambda$, which allows us to produce a single estimate of $z_{c}$, viz., $z_{c}=2.19 \pm 0.03$ (see main text).

simulation over $2 \times 10^{9}$ Monte Carlo moves per lattice site, using the shifting time window method.

For each value of $\lambda$, three different system sizes are used: $L=40,80,160$. For $L=40$, the simulation runs for about 5 hours, and it takes about 3 days to obtain the results for $L=160$.

At short times $(t \approx 1)$, the individual changes of $\Phi$ are uncorrelated; i.e., the meansquare displacement (MSD) of the order parameter must behave as $\left\langle\Delta \Phi^{2}(t)\right\rangle \sim L^{d} t$, where $d=2$ is the spatial dimension of the system.

At long times, $t \gtrsim L^{z_{c}}$, we expect $\langle\Phi(t) \Phi(0)\rangle=0$, which means that

$$
\left\langle\Delta \Phi^{2}(t)\right\rangle \underset{t \gg L^{z_{c}}}{\approx} 2\left\langle\Phi(t)^{2}\right\rangle \sim L^{d+\gamma / \nu},
$$

which is an equilibrium quantity.

If we assume that the MSD is given by a simple power-law in the intermediate time regime $\left(1 \lesssim t \lesssim L^{z_{c}}\right)$, then we have

$$
\left\langle\Delta \Phi^{2}(t)\right\rangle \sim t^{c}
$$

where $c=\gamma /\left(\nu z_{c}\right)$. Note that exactly the same behavior has been found in the Ising model $[28,74]$.

In order to measure the value of the exponent $c$ from $\left\langle\Delta \Phi^{2}(t)\right\rangle$, we need to focus on the intermediate time regime, i.e. we consider the MSD data in $\left(t_{\min }, t_{\max }\right)$ to estimate the exponent. From these data we calculate the exponent $c$ as numerical derivative as $c=\frac{1}{t_{\max }-t_{\min }} \sum_{t=t_{\min }}^{t_{\max }-1} \frac{\ln \left\langle\Delta \Phi^{2}(t+1)\right\rangle-\ln \left\langle\Delta \Phi^{2}(t)\right\rangle}{\ln (t+1)-\ln t}$. In order to estimate $z_{c}$ for different $\lambda$ from these data, we use the data from the largest system size so that we can limit the influence of finite-size effects. From the numerically obtained $c$ we calculate $z_{c}$ and $c=\gamma /\left(\nu z_{c}\right)$, which we present in Table. 4.3. These results, too, indicate that the value of $z_{c}$ is likely independent of $\lambda$. If we do assume that, then we can combine the different numerical values for different $\lambda$ to produce a single estimate of $z_{c}$, viz., $z_{c}=2.19 \pm 0.03$.

The corresponding data for the MSD of $\Phi(t)$ for $80 \leq L \leq 160$ for different values of $\lambda$ are shown in Figure 4.2. The small deviation in Figure 4.2 at late times is caused by periodic boundary conditions: they are different when free boundary conditions are 
Critical Dynamical Exponent of the Two-Dimensional Scalar $\phi^{4}$-Model with Local Moves
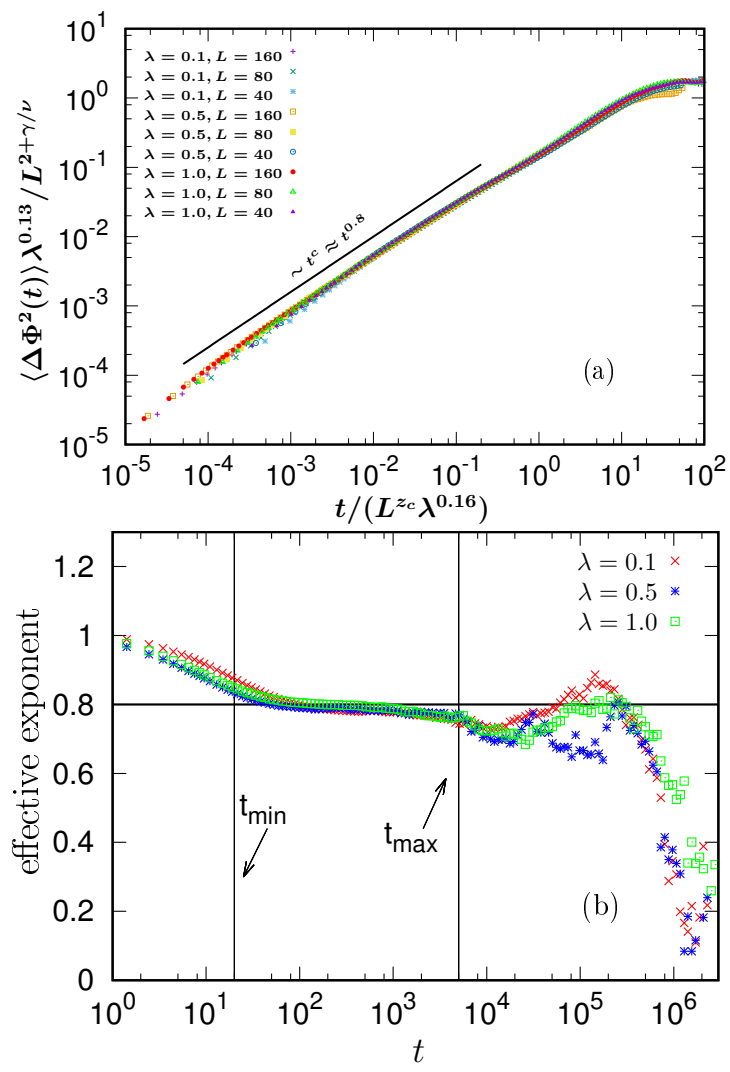

Figure 4.2: (a) The mean-square displacement (MSD) of the order parameter $\left\langle\Delta \Phi^{2}(t)\right\rangle$ in the $\phi^{4}$-model at the critical point. The MSD scales as $\left\langle\Delta \Phi^{2}(t)\right\rangle \sim L^{2} t^{c}$ where $c=\gamma /\left(\nu z_{c}\right)$. The $x$ and $y$ axes are scaled with $\lambda$-dependent numerical scale factors to achieve good quality data collapse for different $\lambda$. The solid line denotes the power-law behavior shown in Eq. (4.5). (b) The effective exponents, i.e., the numerically differentiated $d \ln \left(\left\langle\Delta \Phi^{2}(t)\right\rangle\right) / d \ln (t)$, of the MSD of the order parameter for different $\lambda$. The system sizes are $L=160$ and the time period for calculating the exponent $c$ is $t \in\left(t_{\text {min }}, t_{\text {max }}\right)$, where $t_{\text {min }} \sim 1$ and $t_{\text {max }} \sim L^{z_{c}}$. 
utilized. (Exactly the same effect has been observed in our earlier work on the Ising model [74]. Verification of the boundary effects is therefore not shown here, since the deviations from the power-law do not scale with $L$, and consequently are not relevant in the scaling limit.)

In conclusion, the critical dynamical exponent $z_{c}$ obtained with two independent methods demonstrate that $z_{c}=2.17 \pm 0.03$ or $z_{c}=2.19 \pm 0.03$ for different values of $\lambda$ in the 2D scalar $\phi^{4}$-model. Both results are consistent with the value of $z_{c}$ for the $2 \mathrm{D}$ Ising model $(2.1665 \pm 0.0012)$. In other words, our results indicate that $z_{c}$ is independent of $\lambda$, and is likely identical to that for the 2D Ising model.

\subsection{The GLE formulation of the anomalous diffusion in the $\phi^{4}$-model}

In Sec. 4.2.3 we numerically obtained that, in the intermediate time regime, the MSD of the order parameter in the $\phi^{4}$-model behaves as

$$
\left\langle\Delta \Phi^{2}(t)\right\rangle \sim L^{2} t^{0.80}
$$

This means that, at the critical point, the order parameter exhibits anomalous diffusion. The same behavior has been observed in the Ising model [28]. The physics of anomalous diffusion in the Ising model has been thoroughly analysed in Ref. [74], where it has also been demonstrated that the physics is identical to that for polymeric systems [53, 54, 55, 62, 63, 64, 66, 56].

Both in the Ising model and polymeric systems, the anomalous diffusion stems from time-dependent restoring forces which lead to the GLE formulation. Translated to the $\phi^{4}$-model, the physics of the restoring force can be described as follows.

Imagine that the order parameter locally changes by an amount $\delta \phi$ due to thermal fluctuations at $t=0$. Due to the interactions among the spins dictated by the Hamiltonian, the system will react to the change in $\delta \phi$. This reaction will be manifest in the two following ways: $(a)$ the system will to some extent adjust to the change of $\delta \phi$, however it will take some time, and $(b)$ during this time the order parameter will also readjust to the persisting value of $\Phi$, undoing at least part of $\delta \phi$. It is the latter that we interpret as the result of inertia that resists change in $\Phi$, and the resistance itself acts as the restoring force to the changes in the order parameter.

\subsubsection{The GLE formulation for the anomalous diffusion in the $\phi^{4}$ - model}

In the Ising model and polymeric systems, the restoring force has led to the GLE description for the anomalous diffusion [74, 53, 54]. We now import that for the $\phi^{4}$-model, with a time-dependent memory function $\mu(t)$ arising out of the restoring forces. The GLE formulation for the anomalous diffusion is described as

$$
\zeta \dot{\Phi}(t)=f(t)+q_{1}(t)
$$


Critical Dynamical Exponent of the Two-Dimensional Scalar $\phi^{4}$-Model with Local Moves

$$
f(t)=-\int_{0}^{t} d t^{\prime} \mu\left(t-t^{\prime}\right) \dot{\Phi}\left(t^{\prime}\right)+q_{2}(t) .
$$

Here $f(t)$ is the internal force, $\zeta$ is the "viscous drag" on $\Phi(t), \mu\left(t-t^{\prime}\right)$ is the memory kernel, $q_{1}(t)$ and $q_{2}(t)$ are two noise terms satisfying $\left\langle q_{1}(t)\right\rangle=\left\langle q_{2}(t)\right\rangle=$ 0 , and the fluctuation-dissipation theorems (FDTs) $\left\langle q_{1}(t) q_{1}\left(t^{\prime}\right)\right\rangle \propto \zeta \delta\left(t-t^{\prime}\right)$ and $\left\langle q_{2}(t) q_{2}\left(t^{\prime}\right)\right\rangle \propto \mu\left(t-t^{\prime}\right)$ respectively.

Equation (4.8b) can be inverted to write as

$$
\dot{\Phi}(t)=-\int_{0}^{t} d t^{\prime} a\left(t-t^{\prime}\right) f\left(t^{\prime}\right)+\omega(t) .
$$

The noise term $\omega(t)$ similarly satisfies $\langle\omega(t)\rangle=0$, and the FDT $\left\langle\omega(t) \omega\left(t^{\prime}\right)\right\rangle=a(\mid t-$ $\left.t^{\prime} \mid\right)$. Then $a(t)$ and $\mu(t)$ are related to each other in the Laplace space as $\tilde{a}(s) \tilde{\mu}(s)=1$.

By combining Eq. (4.8a) and (4.8b), we obtain

$$
\zeta \dot{\Phi}(t)=-\int_{0}^{t} d t^{\prime} \mu\left(t-t^{\prime}\right) \dot{\Phi}\left(t^{\prime}\right)+q_{1}(t)+q_{2}(t) .
$$

or

$$
\dot{\Phi}(t)=-\int_{0}^{t} d t^{\prime} \theta\left(t-t^{\prime}\right)\left[q_{1}(t)+q_{2}(t)\right]
$$

where in the Laplace space $\tilde{\theta}(s)[\zeta+\tilde{\mu}(s)]=1$. With $t>t^{\prime}$, without any loss of generality, using Eq. (4.11) the result of the velocity autocorrelation is

$$
\langle\dot{\Phi}(t) \dot{\Phi}(0)\rangle \sim \theta\left(t-t^{\prime}\right)
$$

where $\theta(t)$ can be calculated by Laplace inverting the relation $\tilde{\theta}(s)[\zeta+\tilde{\mu}(s)]=1$.

If $\mu(t)$ behaves as a power-law in time with an exponential cutoff such as

$$
\mu(t) \sim L^{-2} t^{-c} \exp (-t / \tau)
$$

then we have [55]

$$
\left\langle\dot{\Phi}(t) \dot{\Phi}\left(t^{\prime}\right)\right\rangle=-\theta\left(t-t^{\prime}\right) \sim-L^{2}\left(t-t^{\prime}\right)^{c-2} \quad \text { for } \quad t \leq \tau \text {. }
$$

By integrating Eq. (4.14) twice in time (the Green-Kubo relation), we obtain

$$
\left\langle\Delta \Phi^{2}(t)\right\rangle \sim L^{2} t^{c} \quad \text { for } \quad t \leq \tau .
$$

The form $\mu(t) \sim L^{-2} t^{-c}$ not only obtains the anomalous exponent for the meansquare displacement, but also the correct $L$-dependent prefactor to achieve the data collapse in Figure 4.2, i.e., if $\mu(t) \sim L^{-2} t^{-c}$, then $\left\langle\Delta \Phi^{2}(t)\right\rangle \sim L^{2} t^{c}$. 


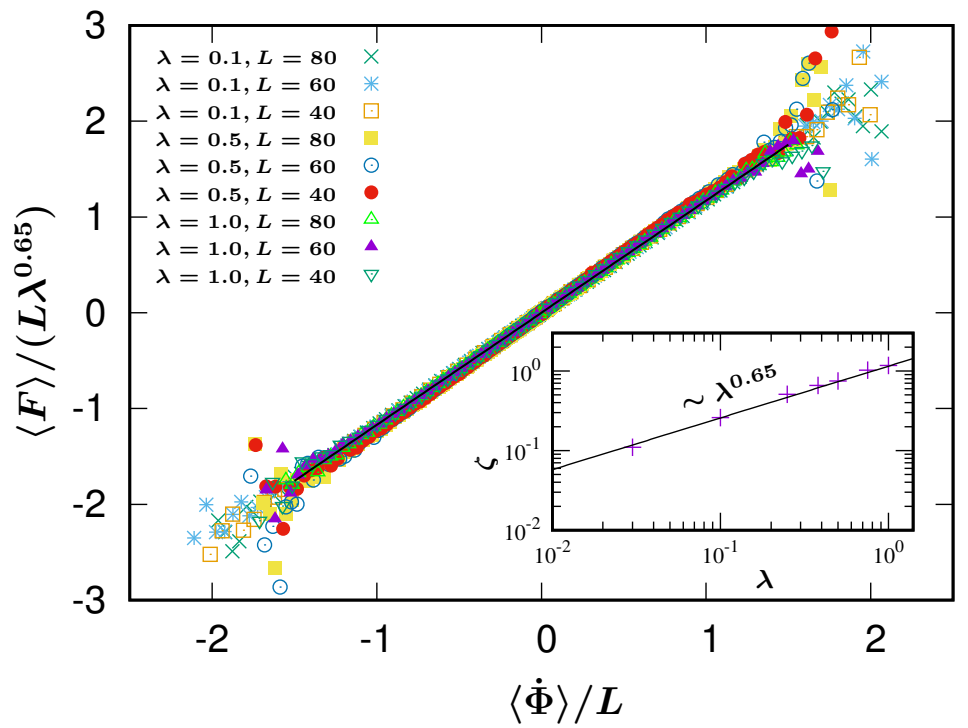

Figure 4.3: The linear relation Eq. (4.8a) between the average internal force $\langle f\rangle$ and $\langle\dot{\Phi}\rangle$ for different $\lambda$. Numerically, we find (inset) that the viscous $\operatorname{drag} \zeta$ behaves as $\zeta \sim \lambda^{0.65}$.

\subsubsection{Verification of the first equation of the GLE and the power-law behavior of $\mu(t)$}

We now numerically verify our proposed GLE formulation, including the form of $\mu(t)$ as stated in Eq. (4.12) for anomalous diffusion in the $\phi^{4}$-model.

First, in order to verify Eq. (4.8a), note that in the $\phi^{4}$-model, the force within the system can be directly calculated as

$$
f=-\frac{1}{L^{2}} \sum_{i=0}^{N} \frac{\partial \mathcal{S}}{\partial \phi_{i}} \frac{\partial \phi_{i}}{\partial \Phi}=-\frac{1}{L^{2}} \sum_{i=0}^{N} \frac{\partial \mathcal{S}}{\partial \phi_{i}} .
$$

By taking ensemble averages on both sides of Eq. (4.8a) we obtain

$$
\langle f(t)\rangle=\zeta\langle\dot{\Phi}\rangle .
$$

This linear relation is demonstrated in Figure 4.3. Additionally, in the inset we plot the viscous $\operatorname{drag} \zeta$ as a function of $\lambda$, and numerically obtain $\zeta \sim \lambda^{0.65}$.

Next we verify the power-law behavior of $\mu(t)$ (Eq. (4.12)) following the FDT $\left.\left\langle f(t) f\left(t^{\prime}\right)\right\rangle\right|_{\dot{\Phi}=0}=\mu\left(t-t^{\prime}\right)$.

We start with a thermalised system at $t=0$. For $t>0$ we fix the value of $\Phi$ (without freezing the whole system), which we achieve by performing non-local spinexchange moves, i.e., at each move, we choose two lattice sites $i$ and $j$ at random, and attempt to change the spin values to $\phi_{i}^{\prime}=\phi_{i}+\Delta \phi$ and $\phi_{j}^{\prime}=\phi_{j}-\Delta \phi$. We calculate 
Critical Dynamical Exponent of the Two-Dimensional Scalar $\phi^{4}$-Model with Local Moves

the change in the energy $\Delta \mathcal{S}$ before and after every attempted move, and accept or reject the move with the Metropolis acceptance probability. While performing spinexchange dynamics, we keep taking snapshots of the system at regular intervals, and compute, at every snapshot (denoted by $t$ ), the force $f(t)$ from Eq. (4.16).

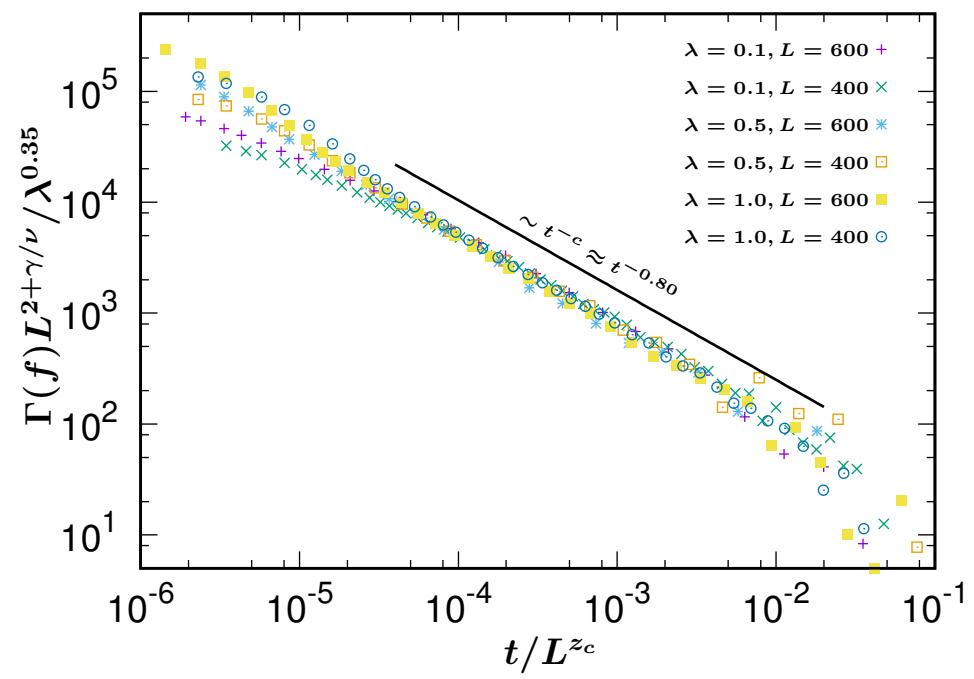

Figure 4.4: Behavior $\Gamma(f) \sim t^{-0.8}$ for different $\lambda$ in the intermediate time regime following Eq. (4.19), then following the FDT, we have $\mu(t) \sim t^{-0.8}$. The extra $\lambda$ dependent factor $\lambda^{0.35}$ is introduced numerically to collapse the data for different $\lambda$ at intermediate times. Further, $z_{c}=2.17$ has been used here to collapse the data.

We notice that since simulations are performed for finite systems with $\Phi$ fixed at its $t=0$ value, we will in any particular run have a non-zero value of $\langle f(t)\rangle$ acting to sustain the initial value of $\Phi[74]$. Thus we calculate the quantity

$$
\Gamma(f)=\left\langle f(t) f\left(t^{\prime}\right)\right\rangle-\langle f(t)\rangle\left\langle f\left(t^{\prime}\right)\right\rangle,
$$

which we expect to represent $\mu\left(t-t^{\prime}\right)$ for all values of $\lambda$, i.e.,

$$
\Gamma(f) \sim L^{-2} t^{-c} \approx L^{-2} t^{-0.80} .
$$

The relation (4.19) is verified in Figure 4.4.

\subsection{Conclusion}

In this chapter, we have measured the critical dynamical exponent $z_{c}$ in the $\phi^{4}$-model using two independent methods: (a) by calculating the relative terminal exponential decay time $\tau$ for the correlation function $\langle\Phi(t) \Phi(0)\rangle$, and thereafter fitting the data as $\tau \sim L^{z_{c}}$, and (b) by measuring the mean-square displacement (MSD) of the order 
parameter $\left\langle\Delta \Phi^{2}(t)\right\rangle \sim t^{c}$ with $c=\gamma /\left(\nu z_{c}\right)$, and $z_{c}$ is calculated from the numerically obtained value $c \approx 0.80$. For different values of the coupling constant $\lambda$, we report that $z_{c}=2.17 \pm 0.03$ and $z_{c}=2.19 \pm 0.03$ for these two methods respectively. Our results indicate that $z_{c}$ is independent of $\lambda$, and is likely identical to that for the $2 \mathrm{D}$ Ising model.

Further, the numerical result $\left\langle\Delta \Phi^{2}(t)\right\rangle \sim t^{0.80}$ at the critical point means that $\Phi(t)$ undergoes anomalous diffusion. We have argued that the physics of anomalous diffusion in the $\phi^{4}$-model at the critical point is the same as for polymeric systems and the Ising model $[53,54,74]$, and therefore a GLE formulation that holds for the Ising model at criticality and for polymeric systems must also hold for the $\phi^{4}$-model. We obtain the force autocorrelation function for the $\phi^{4}$-model at $\dot{\Phi}=0$, and the results allow us to demonstrate the consistency between anomalous diffusion and its GLE formulation. In comparison to the Ising model, since $\Phi$ is a continuous order parameter and there is a proper definition of the internal force, we believe that the $\phi^{4}$-model is a better choice to verify the FDT for the GLE formulation.

Finally, we note that we have confined ourselves to the range $\lambda \in(0,1]$. It is clearly possible to extend our study to larger values of $\lambda$, in particular to $\lambda \rightarrow \infty$, where the model converges to the Ising model, but not without facing additional challenges, as follows. The thermal fluctuations decrease with increasing $\lambda$, and the effective interactions among the fields become weaker [41]. For large $\lambda$, the self-energy term of the fields in the Hamiltonian becomes large. The step size has to be chosen small, otherwise it will lead to many rejected moves. As a consequence, the system gets trapped within narrow bands on the energy landscape. Our preliminary attempts to simulate the model at large $\lambda$ reveal that these traps give rise to artifacts (e.g., in force autocorrelation function at fixed $\Phi$ ) that are not easy to get rid of. These are issues we will explore in the future. 
CHAPTER 5

\section{Super slowing down in the bond-diluted Ising model}

In models in statistical physics, the dynamics often slows down tremendously near the critical point. Usually, the correlation time $\tau$ at the critical point increases with system size $L$ in power-law fashion: $\tau \sim L^{z}$, which defines the critical dynamical exponent $z$. We show that this also holds for the $2 \mathrm{D}$ bond-diluted Ising model in the regime $p>p_{c}$, where $p$ is the parameter denoting the bond concentration, but with a dynamical critical exponent $z(p)$ which shows a strong $p$-dependence. Moreover, we show numerically that $z(p)$, as obtained from the autocorrelation of the total magnetization, diverges when the percolation threshold $p_{c}=1 / 2$ is approached: $z(p)-z(1) \sim\left(p-p_{c}\right)^{-2}$. We refer to this observed extremely fast increase of the correlation time with size as super slowing down. Independent measurement data from the mean-square deviation of the total magnetization, which exhibits anomalous diffusion at the critical point, supports this result. 


\subsection{Introduction}

The Ising model has proven to be a staple model in physics for studying phase transitions and critical phenomena $[85,86]$. The model was originally conceived to provide a theoretical understanding of the existence of a Curie temperature for "pure" ferromagnetic materials; purity here refers to the fact that all throughout the material, every lattice site contains a spin, and every spin interacts uniformly with the surrounding ones. From that point of view, it can be argued that in nature pure materials are rare, i.e., impurities are by and large inevitable.

In the Ising model, the impurities have been implemented in terms of randomly placed nonmagnetic spins (site-diluted Ising model) [87, 88, 89, 90, 91] or missing interactions (bond-diluted Ising model) [92, 93, 94, 95, 96]. The inclusion of any kind of randomness into the system can have significant effects on its critical properties [97]. For instance, a new universality class was found in the three-dimensional bond-diluted Ising model [98, 99], and complex logarithmic corrections for the equilibrium properties were observed [91, 100]. Moreover, a large number of novel crossover behaviors between pure and percolating Ising systems have been found [101, 102, 103, 104, 105, 106]. Also, the dynamics at the percolation threshold is discussed in Refs. [107, 108] and the dynamical exponent for spin systems with random dilution, or randomness in the coupling constants has been considered in Refs. [109, 110, 111, 112, 113]. Despite these advances, dynamical properties of the bond-diluted Ising model (i.e., as a function of bond concentration $p$ ) remains poorly studied.

In this paper, we take on studying the slowing down of the dynamics of the total magnetization autocorrelation function at the critical temperature $T_{c}(p)$ in the square $(L \times L)$ two-dimensional bond-diluted Ising model with Monte Carlo simulations. To this end, using the Binder cumulant, we first measure $T_{c}(p)$ at several values of $p$. We then turn to the calculation of $z(p)$ for several $p>p_{c}$ from the total magnetization autocorrelation function: by collapsing this autocorrelation function to a reference curve, we calculate the relative terminal exponential decay time $\tau\left[T_{c}(p)\right]$ for the correlation function. Thereafter, by fitting this data as $\tau\left[T_{c}(p)\right] \sim L^{z(p)}$, we directly extract $z(p)$. As $p \rightarrow p_{c}^{+}$, we empirically find that the dynamical exponent $z(p)$ increases continuously as $z(p)-z(1) \sim\left(p-p_{c}\right)^{-2}$, with $z(1)=2.1665(12)$ the dynamical critical exponent of the ordinary Ising model [29].

Further, we also consider the mean-square deviation (MSD) of the total magnetization $M$ of the model, which for $p=1$ has been shown to exhibit anomalous diffusion as $\left\langle\Delta M^{2}(t)\right\rangle \sim t^{\alpha}$ with the anomalous exponent $\alpha=\frac{\gamma}{\nu z(1)}$ [74], with $\gamma=7 / 4$ and $\nu=1$. Given that the equilibrium critical exponents $\gamma$ and $\nu$ are numerically nearly independent of $p$ for $p \geq 0.6$ [44], combined with values for $z(p)$ as obtained through the terminal relaxation time for different $p$, the various MSD-curves of the total magnetization are collapsed on top of each other with a $p$-dependent shift factor $\mathcal{G}(p)$ via $\ln \left(\left\langle\Delta M^{2}\right\rangle / L^{2+\gamma / \nu}\right) / \alpha(p) \sim \ln \left(t / L^{z(p)}\right)+\ln (\mathcal{G}(p)) / \alpha(p)$, with $\alpha(p)=\gamma(p) /[\nu(p) z(p)]$. The result reveals that the magnetization indeed experiences anomalous diffusion at the critical point, for a range of dilution $p>p_{c}$. The collapse of the MSD of the magnetization confirms that the measured values of $z(p)$ 
are correct.

The chapter is organized as follows. In Sec. 5.2 we introduce the 2D bond-diluted Ising model and measure its critical temperature at several values of $p$. In Sec. 5.3 we obtain the dynamical exponent $z(p)$ from the total magnetization autocorrelation function. In Sec. 5.4, we confirm $z(p)$ values from the exponent of anomalous diffusion of the MSD of the total magnetization. We conclude the chapter in Sec. 5.5.

\subsection{Bond-diluted Ising model and its critical tempera- ture}

We consider the two-dimensional (2D) bond-diluted Ising model on an $L \times L$ square lattice with periodic boundary conditions. For this model the Hamiltonian, without an external field, is given by

$$
\mathcal{H}=-\sum_{\langle i j\rangle} J_{i j} s_{i} s_{j},
$$

where $s_{i}= \pm 1$ is the spin residing at site $i,\langle i j\rangle$ denotes the sum running over all nearest neighbor sites, and the coupling constant $J_{i j}$ is given by the distribution function

$$
P\left(J_{i j}\right)=p \delta\left(J_{i j}-1\right)+(1-p) \delta\left(J_{i j}\right),
$$

with $p$ being the bond concentration $(0 \leq p \leq 1)$. The function (5.2) simply means that the value of $J_{i j}$ is 1 with probability $p$, and 0 otherwise.

\begin{tabular}{c|c|c|c|c|c|c|c|c|c}
$p$ & 0.9 & 0.85 & 0.8 & 0.75 & 0.7 & 0.65 & 0.6 & 0.58 & 0.55 \\
\hline \hline$N$ & 2000 & 5000 & 20000 & 20000 & 20000 & 20000 & 200000 & 200000 & 400000 \\
\hline$T_{C}$ & $1.956(10)$ & $1.804(10)$ & $1.650(20)$ & $1.472(20)$ & $1.310(25)$ & $1.141(30)$ & $0.951(20)$ & $0.869(25)$ & $0.727(40)$ \\
\hline
\end{tabular}

Table 5.1: Number of samples $N(p)$ used to measure $T_{c}(p)$, and the simulation results for $T_{c}(p)$ (including error bars) for different bond concentrations $p$.

For the pure Ising model $(p=1)$, there is a second-order phase transition at $T_{c}(1)=2 / \ln (1+\sqrt{2})$ [32]. When $p$ reaches the percolation threshold $p_{c}=1 / 2$, its critical temperature decreases to zero: $T_{c}\left(p_{c}\right)=0$ [43]. To determine $T_{c}(p)$ for in-between values of $p$, we use the Binder cumulant. It is defined as [114]

$$
U(T, L)=1-\frac{\left\langle M^{4}\right\rangle}{3\left\langle M^{2}\right\rangle^{2}}
$$

where $\left\langle M^{4}\right\rangle$ and $\left\langle M^{2}\right\rangle$ are the thermal averages of the fourth and second moments of the total magnetization $M=\sum_{i=1}^{L \times L} s_{i}$. For each value of $p$, the curves of $U(T, L)$ plotted vs. $T$ for various values of $L$ intersect at a fixed point, which determines the critical temperature. The process is illustrated in Fig. 5.1(a).

We perform Monte Carlo simulations using the Wolff algorithm [45, 48] to calculate $T_{c}(p)$. Running many independent samples provides us with fairly accurate values 
of these critical temperatures, as noted in Table 5.1. In Fig. 5.1(b) we show that the values for $T_{c}(p)$ obtained this way match very well with those in Refs. [44, 115].
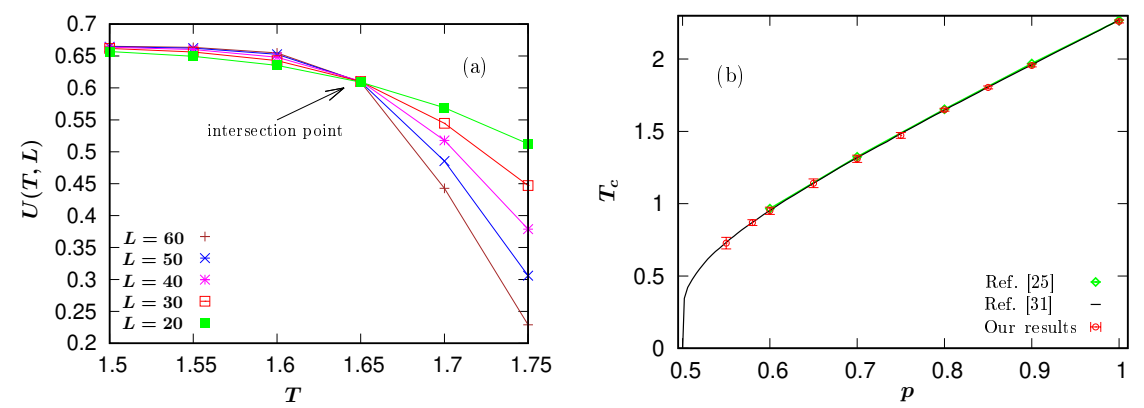

Figure 5.1: (a) Example calculation of $T_{c}(p)$ for the 2D bond-diluted Ising model for $p=0.8$ using the Binder cumulant. The $x$-value of the intersection point indicates that $T_{c}(p=0.8)=1.650 \pm 0.020$. (b) Critical temperatures for different values of $p$, as noted in Table 5.1. Our results match those of Refs. [44, 115] very well.

\subsection{Dynamical exponent for different values of $p$}

Having obtained the critical temperatures for a number of $p$-values as per above, in this section, we measure the total magnetization autocorrelation function $\langle M(t) \cdot M(0)\rangle$. In order to do so, we first run $2 \times 10^{6}$ Wolff Moves to thermalise the system. Subsequently, we evolve the system following Glauber dynamics, i.e. spin flips are proposed at random locations, and accepted with the Metropolis acceptance probability. Time is measured in terms of attempted Monte Carlo moves, since every spin attempts to flip statistically once per unit time. As we continue to do so, we keep taking snapshots of the full system at regular intervals over a total time of $2 \times 10^{7}$ attempted Monte Carlo moves per lattice site, and correspondingly compute the total magnetization $M$ at every snapshot. This leads us to $\langle M(t) \cdot M(0)\rangle$. For different values of $p$, we run 500 to 2000 independent simulations to achieve decent accuracy. We vary the system size from 10 to 40 .

For a given value of $p$ and the corresponding critical temperature $T_{c}(p)$, we collapse all the curves for the normalized total magnetization autocorrelation function $\langle M(t) \cdot M(0)\rangle /\left\langle M(0)^{2}\right\rangle$ to a reference curve $(L=10)$. This allows us to compute the ratio of the terminal decay times $\tau\left[T_{c}(p)\right] / \tau\left[T_{c}(p)\right]_{L=10}$. Fig. 5.2 demonstrates this procedure for $p=0.8$ : with a properly chosen value of $\tau\left[T_{c}(p)\right] / \tau_{L=10}\left[T_{c}(p)\right]$, the $\langle M(t) \cdot M(0)\rangle /\left\langle M(0)^{2}\right\rangle$ data for different system sizes collapse on the curve corresponding to $L=10$.

Further, given our argument in Appendix A that $L$ is the characteristic length scale for $L \geq 10$ for the 2D bond-diluted Ising model when $p \geq 0.6$, we have, at the critical temperature,

$$
\tau(p) / \tau_{L=10}(p) \sim L^{z(p)}
$$




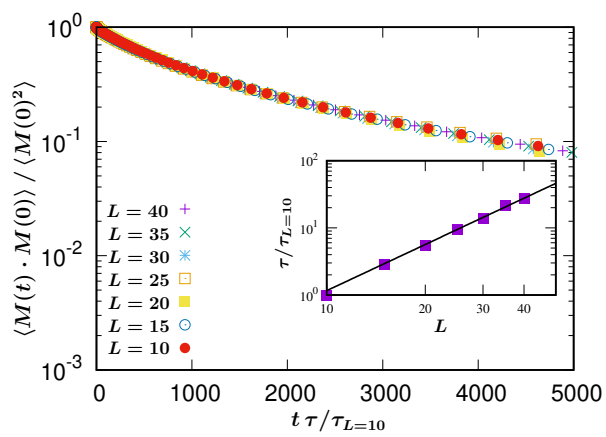

Figure 5.2: The collapse of $\langle M(t) \cdot M(0)\rangle /\left\langle M(0)^{2}\right\rangle$ as a function of $t \tau\left[T_{c}(p)\right] / \tau\left[T_{c}(p)\right]_{L=10}$ for $p=0.8$. The system size varies from 10 to 40 . Inset: correspondingly, $\tau\left[T_{c}(p)\right] / \tau\left[T_{c}(p)\right]_{L=10}$ as a function of $L$. The dynamical exponent is obtained by fitting these data as $\tau\left[T_{c}(p)\right] / \tau\left[T_{c}(p)\right]_{L=10} \sim L^{z(p)}$. The solid line corresponds to the function $y=x^{2.285}$. From this we obtain $z(0.8) \approx 2.285$.

By plotting the $\tau(p) / \tau_{L=10}(p)$ data (inset Fig. 5.2), we extract $z(p)$. The results from this exercise for several values of $p$ are shown in Fig. 5.3. Numerically, therein we find that

$$
\Delta z(p)=z(p)-z(1) \sim\left(p-p_{c}\right)^{-2} \text { for } \quad p_{c}<p<1,
$$

where $z(1)=2.1665(12)$ [29] is the dynamical exponent for the pure 2D Ising model.

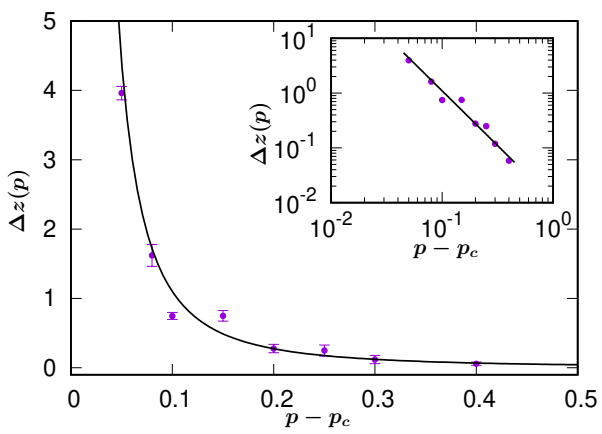

Figure 5.3: The dynamical exponent difference $\Delta z(p)=z(p)-z(1)$ as a function of $p-p_{c}$, where $z(1)=2.1665(12)$ [29] is the dynamical exponent for the pure $2 D$ Ising model. The result implies that $z(p) \rightarrow \infty$ as $p \rightarrow p_{c}^{+}$.

Based on concepts of normalization, we anticipated that away from the percolation threshold $p_{c}$, the correlation time as a function of system size would show a crossover from the behavior for the bond-diluted Ising model at small system sizes to that of the ordinary Ising model at large system sizes, with a crossover size that diverges if $p_{c}$ 
is approached. Instead, we find a fairly clean power-law behavior of the correlation time for all system sizes, with a single exponent $z(p)$ that varies strongly with $p$. As a function of bond dilution $p$, the dynamical exponent $z(p)$ increases monotonically when $p$ decreases from $p=1$ to $p=p_{c}$. Moreover, the numerical results suggest that $z(p)$ will become infinitely large when $p$ approaches the percolation threshold $p \rightarrow$ $p_{c}^{+}$, i.e., the dynamics of the system gets extremely slow as $p \rightarrow p_{c}^{+}$, a phenomenon we term as "super slowing down".

\subsection{Anomalous diffusion of the total magnetization}

To confirm the observed behavior of super slowing down [i.e., the Eq. (5.5)] for the bond-diluted Ising model by means of independent measurements, we now focus on the mean-square deviation (MSD) of the magnetization as a function of time $t$ as

$$
\left\langle\Delta M^{2}\right\rangle=\left\langle[M(t)-M(0)]^{2}\right\rangle
$$

At short times $(t \approx 1)$, changes in $M$, occurring due to random thermal fluctuations of individual spins, are uncorrelated; hence $\left\langle\Delta M^{2}\right\rangle \sim L^{2} t$ for the 2D Ising model. At long times, $t \gtrsim L^{z(p)}$, we expect $\langle M(t) \cdot M(0)\rangle=0$, meaning that

$$
\left\langle\Delta M^{2}\right\rangle \underset{t \gtrsim L^{z(p)}}{=} 2\left\langle M(t)^{2}\right\rangle \sim L^{2+\gamma(p) / \nu(p)} .
$$

If we assume that the MSD is given by a simple power law in the intermediate time regime $\left(1 \gtrsim t \gtrsim L^{z(p)}\right)$, then we obtain

$$
\left\langle\Delta M^{2}\right\rangle \sim L^{2+\gamma(p) / \nu(p)}\left(t / L^{z(p)}\right)^{\alpha(p)}
$$

where $\alpha(p)=\frac{\gamma(p)}{\nu(p) z(p)}$. For the pure Ising model in two dimensions $(p=1)$, we have shown that [74]

$$
\left\langle\Delta M^{2}\right\rangle / L^{2+\gamma / \nu}=f\left(t / L^{z}\right),
$$

where $\gamma=7 / 4$ and $\nu=1$ are two equilibrium critical exponents. Here $f(x)$ is a scaling function such that $\lim _{x \rightarrow 0} f(x) \sim x^{\gamma /(\nu z)} \approx x^{0.81}$, and $f(x)$ saturates for $x \gtrsim 1$. Indeed, given that $\gamma(p)$ and $\nu(p)$ are nearly independent of $p$ when $p \geq 0.6$ [44] (see also in Appendix B), if the scaling relation (5.9) also continues to hold for values of $p$ other than unity, then we can use it to obtain independent confirmation for the super slowing down (5.5). We demonstrate this below by focusing on $p \geq 0.6$.

Since in the previous section, we have obtained the values of $z(p)$ for different $p$, here, we describe the MSD of the total magnetization by modifying Eq. (5.8) as

$$
\left\langle\Delta M^{2}\right\rangle / L^{2+\gamma / \nu} \sim \mathcal{G}(p)\left(t / L^{z(p)}\right)^{\alpha(p)}
$$




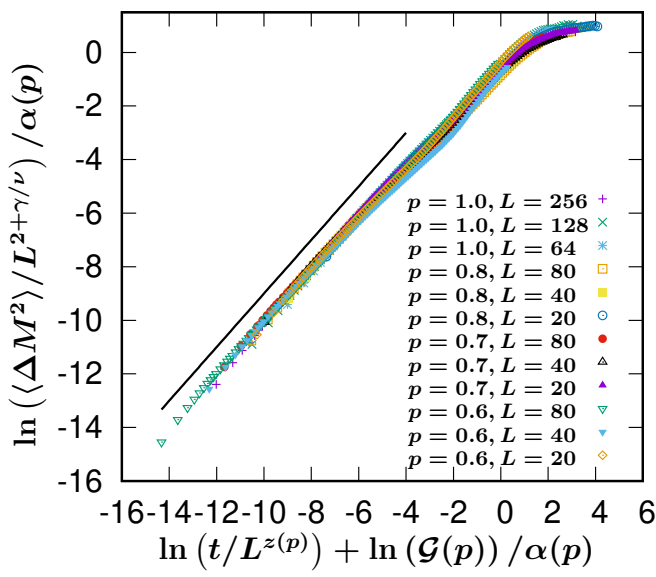

Figure 5.4: The collapse of the mean-square displacement of the total magnetization via $\ln \left(\left\langle\Delta M^{2}\right\rangle / L^{2+\gamma / \nu}\right) / \alpha(p) \sim \ln \left(t / L^{z(p)}\right)+\ln (\mathcal{G}(p)) / \alpha(p)$, where the obtained values of $z(p)$ from the last section are employed and $\mathcal{G}(p)$ is a $p$-dependent shift factor. The slope of the solid line is unity. It confirms that the MSD of the total magnetization experiences anomalous diffusion at $T_{c}(p)$ and the value of $z(p)$ is increasing when $p \rightarrow p_{c}+$.

where $\mathcal{G}(p)$ is a $p$-dependent shift factor. We take logarithm of both sides of Eq. (5.10) to write

$$
\ln \left(\left\langle\Delta M^{2}\right\rangle / L^{2+\gamma / \nu}\right) / \alpha(p) \sim \ln \left(t / L^{z(p)}\right)+\ln (\mathcal{G}(p)) / \alpha(p) .
$$

Suppose we choose the MSD of the total magnetization for the normal Ising model as the reference [means that we set $\mathcal{G}(1) \equiv 1$ ], if the values of $z(p)$ obtained from the last section are correct, then with these $z(p)$ values and the shift factor $\mathcal{G}(p)$, the MSD of the total magnetization for different $p$ can be made to collapse onto the data for $p=1$ via Eq. (5.11).

In order to obtain the $\left\langle\Delta M^{2}(t)\right\rangle$ data, once again, we first thermalise the system with $2 \times 10^{6}$ Wolff moves, then measure $\left\langle\Delta M^{2}\right\rangle$ in a further simulation over $2 \times 10^{7}$ attempted Monte Carlo moves per lattice site. We use three different system sizes: $L=20,40,60$ for every value of $p$.

Figure 5.4 implies that by using the values of $z(p)$ obtained from the last section, indeed for different $p$, the MSD of the magnetization can be collapsed onto the data for $p=1$ via Eq. (5.11). It confirms that the MSD of the total magnetization experience anomalous diffusion at $T_{c}(p)$ and $z(p)$ values obtained from the terminal relaxation time are correct.

In summary, with two different methods, we have shown that $z(p)$ is diverging when $p \rightarrow p_{c}^{+}$, i.e., the dynamics of the system is getting extremely slow when we reduce the bond concentration to its percolation threshold. We do not have a quantitative explanation for this behavior. That said, it might arise from the fact that the 
fraction of 'unhappy' bonds (active bonds between sites with opposing spin values) at the critical temperature decreases to zero if $p_{c}$ is approached, thereby removing the energetic contribution of restoring forces; we provide some measurements for this in Appendix C.

\subsection{Discussion}

In this paper, we study the critical dynamical exponent $z(p)$ for the $2 \mathrm{D}$ bond-diluted Ising model with bond concentration $p$. We first measure the critical temperature $T_{c}(p)$ for different bond concentrations $p$ using the Binder cumulant. We then calculate the relative values of the terminal decay time $\tau$ by collapsing the total magnetization autocorrelation function to a reference value, from which we obtain $z(p)$ using the relation $\tau \sim L^{z(p)}$.

We find that $z(p)$ increases when $p \rightarrow p_{c}^{+}$as a power-law $z(p)-z(1) \sim\left(p-p_{c}\right)^{-2}$, which we refer to as super slowing down. We confirm this result from independent measurements of the MSD of the total magnetization that exhibits anomalous diffusion.

Our results indicate that $z(p) \rightarrow \infty$ as $p \rightarrow p_{c}^{+}$. This leaves us with the interesting question: what happens to $z(p)$ when $p<p_{c}$ ? We plan to explore this in the future.

\subsection{Appendixes}

\subsubsection{Appendix A: Relevant length scale for critical phenomena of the bond-diluted Ising model}
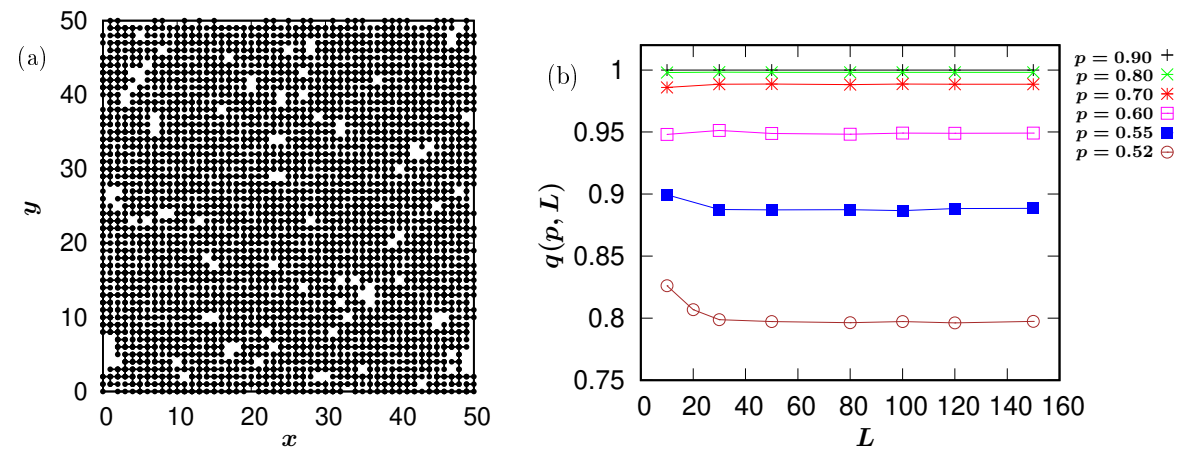

Figure 5.5: (a) A snapshot of the biggest knot cluster for $L=50$ and $p=0.6$. Spins that do not belong to this cluster are represented by cavities. (b) Plot of $q(p, L)=$ $\langle S(p, L)\rangle / L^{2}$ for various values of $p$ and $L$ : for $p \geq 0.6, q(p, L)$ is independent of $L$ for $L \geq 10$. 
In the pure $2 \mathrm{D}$ Ising model of dimension $L \times L$, the only relevant length scale for critical phenomena is $L$. For the bond-diluted Ising model there are other length scales, for instance corresponding to the size of the biggest cluster, $S(p, L)$. Here, a cluster is defined as the set of spins such that there is at least one continuous (bondfollowing) path from every spin in the cluster to every other spin in the same cluster. We define the size of the cluster by the total number of spins belonging to the cluster [thus $S(p, L)$ is the number of spins in the biggest cluster for an $L \times L$ system with bond concentration $p$ ].

In Fig. 5.5(b) the quantity considered is $q(p, L)=\langle S(p, L)\rangle / L^{2}$. If this quantity is independent of $L$ then it means that there is no difference between the two differently defined length scales (apart from a scaling factor). For each result, we have generated 500 samples. We see in Fig. 5.5(b) that for $p \geq 0.6, q(p, L)$ is independent of $L$ for $L \geq 10$. This means that for the range of dilution $p \geq 0.6$ used in this paper, we can use $L$ as the relevant length scale for critical phenomena provided $L \geq 10$.

\subsubsection{Appendix B: Equilibrium critical exponents $\nu$ and $\gamma$}

In this Appendix, we show that the equilibrium critical exponents $\nu$ and $\gamma$ for the bond-diluted Ising system with $p \geq 0.6$ are numerically indistinguishable from their values in the pure Ising model.

We note here that according to the Harris criterion [116], if the correlation length critical exponent $\nu$ fulfills the inequality $\nu \geq 2 / d$ where $d$ is the spatial dimensionality, then disorder does not affect the critical behavior. For the $2 D$ Ising model, $\nu=1$ is marginal, which translates into logarithmic corrections to some critical exponents. In the pure Ising model, the exponents $\gamma$ and $\nu$ do not show logarithmic corrections, and our numerical results shown in this Appendix indicate that the ratio of $\gamma$ and $\nu$ is unchanged in the regime we studied, for $p \geq 0.6$, without logarithmic corrections. Also, the Binder cumulant does not show logarithmic corrections. This is not obvious, and in fact there are reports of logarithmic corrections to the equilibrium properties of the diluted spin systems $[91,117,118]$. We cannot rule out the possibility to have logarithmic corrections in the quantities measured by us, as these are difficult to observe in simulations.

Firstly, if we get the values of $T_{c}$ for different $p$, the Binder cumulant can be scaled as

$$
U(T, L) \sim f\left(T^{\prime} L^{1 / \nu(p)}\right),
$$

which will provide us the value of $\nu(p)$. Here $T^{\prime}=\left(T-T_{C}\right) / T_{C}$ is the reduced temperature.

In Fig. 5.6, we collapse the data of $U(L, T)$ for $L=20,40$ and 60 with $\nu(p) \approx 1$. It indicates that $\nu(p)$ is numerically indistinguishable from unity for $p \geq 0.6$.

Next, we turn to measure the magnetic susceptibility $\chi$. For this simulation, we have used 500 independent samples for each value of $p$. It is a well known result [45] that the susceptibility can be scaled as

$$
\chi L^{-\gamma / \nu}=\tilde{\chi}\left(T^{\prime} L^{1 / \nu}\right),
$$




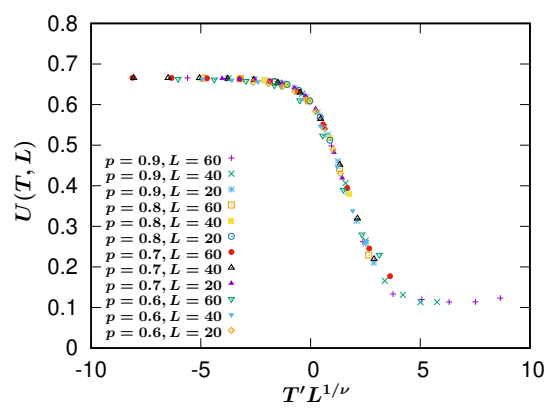

Figure 5.6: The Binder cumulant for $p \geq 0.6$. The data is collapsed as $U(T, L) \sim$ $f\left(T^{\prime} L^{1 / \nu(p)}\right)$ for different $p$ and $L$, where $T^{\prime}=\left(T-T_{C}\right) / T_{C}$ is the reduced temperature. Here $L=20,40$ and 60 for each bond concentration, and we set all $\nu(p)=1.0$ to collapse the data.

where $\tilde{\chi}$ is a dimensionless function.

After rescaling the susceptibility using Eq. (5.13), the data shown in Fig. 5.7 demonstrate that $\gamma$ is numerically indistinguishable from $7 / 4$ for $p \geq 0.6$.

In other words, in this Appendix we have shown that $\gamma$ and $\nu$ are numerically indistinguishable respectively from $7 / 4$ and unity for $p \geq 0.6$, confirming the results from Ref. [44].

\subsubsection{Appendix C: Number of different types of bonds}

In this Appendix, we connect the super slowing down in the $2 D$ bond-diluted Ising model with its equilibrium property, i.e., the ensemble average of the number of 'unhappy' bonds, i.e. the number of interacting nearest-neighbor spins with opposite signs at the critical temperature.

In the bond-diluted Ising model, we distinguish inactive bonds (with $J_{i j}=0$ ), active bonds connecting sites with aligned spins, and active bonds that connect sites with spins of opposite signs. For the active bonds, we denote the numbers of those aligned and nonaligned spins by $\left(n_{++}+n_{--}\right)$and $n_{+-}$, respectively. Energetically, $\left\langle n_{++}\right\rangle$and $\left\langle n_{--}\right\rangle$are the bonds that try to keep the system as it is, and $\left\langle n_{+-}\right\rangle$is driving spins to flip. If $\left\langle n_{+-}\right\rangle$decreases, then most of the proposed spin flips will be rejected and the dynamics of the system will get slower.

In our simulations, we have performed 100 independent samples to obtain the number of 'unhappy' bonds. The measured values of $\left\langle n_{+-}\right\rangle$at the critical temperature can be found in Fig. 5.8, with a log-log plot as an inset. In particular, numerically we find that

$$
\left\langle n_{+-}\right\rangle / L^{2} \sim\left(p-p_{c}\right)^{0.97 \pm 0.03} \text { for } p \geq p_{c} .
$$

When $p \rightarrow p_{c}^{+}$, the values of $\left\langle n_{+-}\right\rangle$reduce to zero (or a value close to zero), which means that most of the active bonds are 'happy' so that spins are unlikely to 

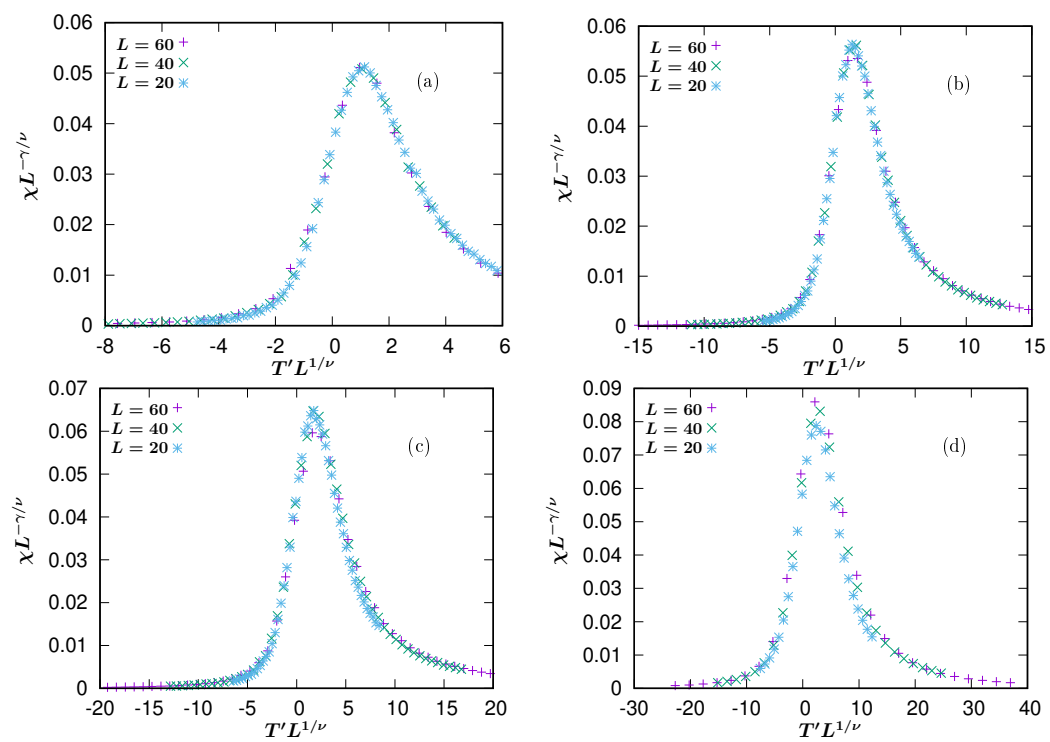

Figure 5.7: The scaling of magnetic susceptibility as a function of reduced temperature: $\chi L^{-\gamma / \nu}=\tilde{\chi}\left(T^{\prime} L^{1 / \nu}\right)$. Here $\tilde{\chi}$ is a dimensionless function, the values of $\gamma(p)$ and $\nu(p)$ are chosen to be their values for the normal Ising model, i.e., $\gamma(p)=1.75$ and $\nu(p)=1$. For figures (a)-(d), the bond concentrations are $p=0.9,0.8,0.7$ and 0.6. The good collapse of all the data indicates that both $\gamma$ and $\nu$ are numerically indistinguishable for $p \geq 0.6$.

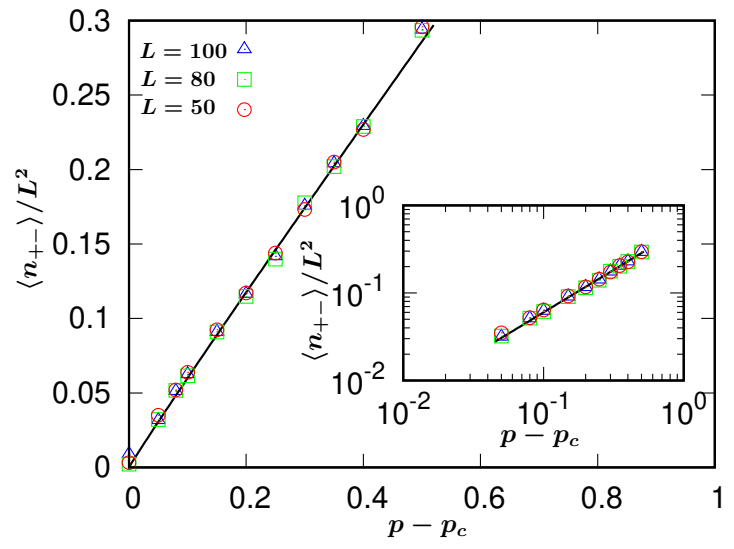

Figure 5.8: The value of $\left\langle n_{+-}\right\rangle$as a function of $p-p_{c}$ for $L=50,80$ and 100. The solid line goes as $\sim\left(p-p_{c}\right)^{0.97}$. The inset is a log-log plot of the data. It suggests that when $p \rightarrow p_{c}^{+},\left\langle n_{+-}\right\rangle \rightarrow 0$, then most of the bonds are activated so that nearly all spins are unlikely to flip, resulting in the super slow dynamics of the system. 
flip. This might explain why the system is getting super slow when $p$ approaches the percolation threshold. 
CHAPTER 6

\section{Approximate dynamical eigenmodes of the Ising model with local spin-exchange moves}

We establish that the Fourier modes of the magnetization serve as the dynamical eigenmodes for the two-dimensional Ising model at the critical temperature with local spin-exchange moves, i.e., Kawasaki dynamics. We obtain the dynamical scaling properties for these modes, and use them to calculate the time evolution of two dynamical quantities for the system, namely the autocorrelation function and the meansquare deviation of the line magnetization. At intermediate times $1 \lesssim t \lesssim L^{z_{c}}$, where $z_{c}=4-\eta=3.75$ is the dynamical critical exponent of the model, we find that the line magnetization undergoes anomalous diffusion. Following our recent work on anomalous diffusion in spin models, we demonstrate that the Generalized Langevin Equation (GLE) with a memory kernel consistently describes the anomalous diffusion, verifying the corresponding fluctuation-dissipation theorem with the calculation of the force autocorrelation function. 


\subsection{Introduction}

For physical systems in statistical physics, the eigenvalues and eigenvectors (of the Hamiltonian) play a central role. The eigenvectors form a complete orthogonal basis in the space of variables used to express the Hamiltonian. The eigenvalues and eigenfunctions identify the ground and the excited states, as well as their energies, which then form the groundwork for obtaining the partition function, the principal quantity of interest for calculating all equilibrium ensemble-averaged observable.

For classical systems, the Hamiltonian also dictates the dynamics of systems through the equations of motion. Here too, theoretically, the same concept holds, viz. with the equation of motion of a degree of freedom $q$ used to describe a Hamiltonian $\mathcal{H}$ being given by

$$
\zeta \dot{q}=-\frac{\partial \mathcal{H}}{\partial q}
$$

with $\zeta$ being the friction coefficient in the overdamped limit, it really is an asset to know the dynamical eigenvalues and eigenvectors. Together, the dynamical eigenvalues and eigenvectors ensure that the full time-dependence of any dynamical quantity can be calculated exactly.

In contrast to eigenvalues and eigenvectors of the Hamiltonian itself, the scope for dynamical eigenvalues and eigenvectors is far more restricted, for the following reason. The eigenvectors $\left\{r_{\alpha}\right\}$ are linear combinations of all the degrees of freedom $\left\{q_{i}\right\}$, reducing Eq. (6.1) to the form

$$
\zeta_{\alpha} \dot{r}_{\alpha}=-\lambda_{\alpha} r_{\alpha}
$$

with $\lambda_{\alpha}$ being the corresponding dynamical eigenvalue, obtained from the diagonalisation of the Hessian matrix $\frac{\partial^{2} \mathcal{H}}{\partial r_{\beta} \partial r_{\gamma}}$. The dynamical eigenmodes $\left\{r_{\alpha}\right\}$, if they exist, are often simply called the modes of the system. For the form (6.2) to hold, the Hessian must be independent of $\left\{r_{\alpha}\right\}$, which restricts the class of such Hamiltonian only to harmonic ones (i.e., $\mathcal{H}$ is quadratic in $\left\{q_{i}\right\}$ ). Classic examples of such systems are the bead-spring models of linear polymeric systems [119, 120], their extensions to star and tadpole polymers [59], polymeric membranes [59, 121], 2D cytoskeleton of cells $[122,123,124]$ and graphite oxide sheets [124, 125, 126, 127].

Not all is however lost if the Hamiltonian is not harmonic (which is in fact almost always the case). Note here that any complete orthogonal basis in the space of the degrees of freedom can be used to describe the dynamics of the system. The main disadvantage of choosing an arbitrary one is that the corresponding amplitudes remain dynamically (nonlinearly) coupled at all times, preventing one from taking large timesteps in computer simulations. Despite this shortcoming, sometimes one can be lucky to realize that there are approximate modes that can allow one to take somewhat large time-steps within a preordained error margin. Examples are the Rouse modes for selfavoiding polymers [56], a reptating polymer chain [128], and polymer chains in a melt $[129,130,131]$. 
Approximate dynamical eigenmodes of the Ising model with local spin-exchange moves

The focus of the present chapter are the (approximate dynamical) modes of the two-dimensional (2D) square-lattice Ising model (system size $L \times L$ ) with local spin exchange moves - commonly known as Kawasaki moves [50] — at critical temperature and at zero order parameter, introduced in Sec. 6.2.1. We focus on the line magnetization for this model and find, surprisingly, that the Fourier modes provide a very good approximation of the true dynamical eigenmodes. We numerically investigate the properties of these modes in Sec. 6.2.2-6.2.4, numerically revealing that the equilibrium amplitude of the $p$-th mode behaves as $(L / p)^{\gamma / \nu}\left(B_{0}+B_{1} p^{-1.75}\right)$, and that its decay time scales $\sim(L / p)^{z_{c}}$, where $\gamma=7 / 4, \nu=1$ and $\eta=2-$ $\gamma / \nu=1 / 4$ are the three equilibrium critical exponents of the Ising model, and $z_{c}=4-\eta=15 / 4$ is the critical dynamical exponent for the model with local spin exchange moves $[132,133,134]$. In Sec. 6.3 we use these results to analytically calculate two observables: the autocorrelation function, and the mean-square deviation (MSD), of the line magnetization. We find that line magnetization exhibits anomalous diffusion. Our results for anomalous diffusion are consistent with a pattern that the dynamics of magnetization at the critical temperature in spin models is anomalous [28, 74, 76]. Importantly, the anomalous diffusion is described by the Generalized Langevin Equation (GLE) [74, 76] (and bears strong resemblance to anomalous diffusion in polymeric and membrane systems under a variety of circumstances $[53,54,55,56,57,58,59,60,61,62,63,64,65,66,67,68]$, which we verify in Sec. 6.4. We conclude the chapter in Sec. 6.5.

\subsection{The model and the Fourier modes as the approxi- mate dynamical modes}

\subsubsection{Ising model with local spin-exchange (Kawasaki) dynamics}

We consider the two-dimensional (2D) Ising model on an $L \times L$ square lattice with periodic boundary conditions in both $x$ - and $y$-directions. The Hamiltonian for the model is given by

$$
\mathcal{H}=-J \sum_{\langle(j, k)(m, n)\rangle} s_{j, k} s_{m, n},
$$

where $s_{j, k}= \pm 1$ is the spin value at $x$-location $j$ and $y$-location $k$, and $J$ is the coupling constant for interactions among the spins and we set $J=1$ during our simulations. The summation runs over all the nearest-neighbor spins, and $0 \leq(j, k, m, n)<$ $L$. All properties we report here have been obtained by simulating the model at the critical temperature $T_{c}=2 / \ln (1+\sqrt{2})$, and by setting the value of the Boltzmann constant $k_{B}$ to unity.

The model is simulated with Kawasaki dynamics at $T_{c}$. All simulations reported in this chapter have been performed at zero (conserved) order parameter. In other words, we fix the total magnetization of the system at zero, and at each Monte Carlo move, two neighboring spins are randomly selected to exchange their values. The resulting energy change $\Delta E$ is measured, and the move is accepted with the normal Metropolis 
probability $\min [1, \exp (-\Delta E / T)]$. For each unit of time, on average, all the spins are supposed to be selected once.

\subsubsection{Fourier modes for line magnetization}

In this model we define the line magnetization as $M_{l}(j, t)=\sum_{k=0}^{L-1} s_{j, k}(t)$; correspondingly, the $p$-th Fourier mode amplitude of the line magnetization is given by

$$
A_{p}(t)=\frac{1}{L} \sum_{j=0}^{L-1} M_{l}(j, t) \exp (-2 \pi i p j / L)=X_{p}(t)-i Y_{p}(t)
$$

where

$$
\begin{aligned}
& X_{p}(t)=\frac{1}{L} \sum_{j=0}^{L-1} M_{l}(j, t) \cos (2 \pi p j / L), \\
& Y_{p}(t)=\frac{1}{L} \sum_{j=0}^{L-1} M_{l}(j, t) \sin (2 \pi p j / L),
\end{aligned}
$$

respectively are the real and the imaginary parts of the Fourier transform, with $p=$ $0,1, \ldots,(L-1)$. The inverse Fourier transform is then given by

$$
\begin{aligned}
M_{l}(j, t) & =\sum_{p=0}^{L-1} A_{p}(t) \exp (2 \pi i p j / L) \quad \text { or } \\
M_{l}(j, t) & =\sum_{p=0}^{L-1}\left[X_{p}(t) \cos (2 \pi p j / L)+Y_{p}(t) \sin (2 \pi p j / L)\right] .
\end{aligned}
$$

\subsubsection{Equilibrium properties of the Fourier mode amplitudes}

We express the equilibrium correlations of the Fourier modes as

$$
X_{p q}(t)=\left\langle X_{p}(t) X_{q}(0)\right\rangle \quad \text { and } \quad Y_{p q}(t)=\left\langle Y_{p}(t) Y_{q}(0)\right\rangle
$$

where the angular brackets $(\langle\cdot\rangle)$ define an average over equilibrated ensembles.

The cross-correlation terms, $\left\langle X_{p}(t) Y_{q}(0)\right\rangle$ and $\left\langle Y_{p}(t) X_{q}(0)\right\rangle$ respectively, can be argued to be equal to zero, as follows. Let us consider $\left\langle X_{p}(t) Y_{q}(0)\right\rangle$ to illustrate the calculation. First, having expressed it as $\sum_{j, m=0}^{L-1}\left\langle M_{l}(j, 0) M_{l}(m, t)\right\rangle \cos (2 \pi p j / L)$ $\sin (2 \pi q m / L)$, then making the simultaneous substitutions $j \rightarrow(L-j)$ and $m \rightarrow$ $(L-m)$, and finally using $M_{l}(0, t)=M_{l}(L, t)$ due to periodic boundary conditions, we find that the term also equals $-\sum_{j, m=0}^{L-1}\left\langle M_{l}(L-j, 0) M_{l}(L-m, t)\right\rangle \cos (2 \pi p j / L)$ 
Approximate dynamical eigenmodes of the Ising model with local spin-exchange moves

$\sin (2 \pi q m / L)$. Next, we use the fact that $\left\langle M_{l}(j, 0) M_{l}(m, t)\right\rangle$ is only a function of $|j-m|$ modulo $L / 2$ (due to periodic boundary conditions) as well as only of $|t|$ (due to time reversibility invariance at equilibrium). This implies that $\left\langle M_{l}(j, 0) M_{l}(m, t)\right\rangle=$ $\left\langle M_{l}(L-j, 0) M_{l}(L-m, t)\right\rangle$, leading to the condition $\left\langle X_{p}(t) Y_{q}(0)\right\rangle=-\left\langle X_{p}(t) Y_{q}(0)\right\rangle=$ 0 . For this reason we leave both $\left\langle X_{p}(t) Y_{q}(0)\right\rangle$ and $\left\langle Y_{p}(t) X_{q}(0)\right\rangle$ out of further considerations.

Next, we argue that $X_{p p}(0)=Y_{p p}(0)$ at least up to $O\left(L^{-2}\right)$. In order to do so, we first express $Y_{p p}(0)$ as

$$
\begin{aligned}
Y_{p p}(0) & =\sum_{j, m=0}^{L-1}\left\langle M_{l}(j, 0) M_{l}(m, 0)\right\rangle \sin (2 \pi p j / L) \sin (2 \pi p m / L) \\
& =\sum_{j, m=0}^{L-1}\left\langle M_{l}(j, 0) M_{l}(m, 0)\right\rangle \cos \left(2 \pi p j^{\prime} / L\right) \cos \left(2 \pi p m^{\prime} / L\right),
\end{aligned}
$$

where $\left(j^{\prime}, m^{\prime}\right)=\left(j+\frac{L}{4 p}, m+\frac{L}{4 p}\right)$. We then again observe, just like in the above paragraph, that $\left\langle M_{l}(j, 0) M_{l}(m, t)\right\rangle$ is only a function of $|j-m|$ modulo $L / 2$. This implies that if $\frac{L}{4 p}$ is an integer, then upon relabeling the line indices the sum trivially reduces to $\sum_{j^{\prime}, m^{\prime}=0}^{L-1}\left\langle M_{l}\left(j^{\prime}, 0\right) M_{l}\left(m^{\prime}, 0\right)\right\rangle \cos \left(2 \pi p j^{\prime} / L\right) \cos \left(2 \pi p m^{\prime} / L\right)=X_{p p}(0)$. If however $\frac{L}{4 p}$ is not an integer, then, we can still relabel the indices as

$$
\sum_{j^{\prime \prime}, m^{\prime \prime}=0}^{L-1}\left\langle M_{l}\left(j^{\prime \prime}, 0\right) M_{l}\left(m^{\prime \prime}, 0\right)\right\rangle \cos \left(2 \pi p\left(j^{\prime \prime}+\Delta x\right) / L\right) \cos \left(2 \pi p\left(m^{\prime \prime}+\Delta x\right) / L\right) \text {, with }
$$

$\Delta x<1,1$ being the lattice unit. Beyond this point, we can do a Taylor expansion of the cosine terms, implying that the equality $Y_{p p}(0)=X_{p p}(0)$ must hold up to $O\left(L^{-2}\right)$. This, together with the scaling of $\left\langle\left|A_{p}^{2}\right|\right\rangle \sim(L / p)^{\gamma / \nu}$ in the limit $p \rightarrow \infty$ for the $2 \mathrm{D}$ Ising model as derived in Appendix A, we attempt to fit $X_{p p}(0)=Y_{p p}(0)$ to the asymptotic scaling $\sim(L / p)^{\gamma / \nu}$ in Figure 6.1.

From this fit, we find that $X_{p p}(0) \approx Y_{p p}(0) \approx(L / p)^{\gamma / \nu}\left(B_{0}+B_{1} p^{-1.75}\right)$, where $B_{0}=0.0185$ and $B_{1}=0.1$ are two numerically obtained constants. Note also that

$$
X_{p(L-q)}(t)=X_{p q}(t) \text { and } Y_{p(L-q)}(t)=Y_{p q}(t),
$$

an obvious result obtained from the symmetry of the mode amplitudes under $p \leftrightarrow$ $L-p$.

The results of Figure 6.1 are supplemented with the data for $\chi_{p q}(0) \equiv X_{p q}(0) /$ $\sqrt{X_{p p}(0) X_{q q}(0)}$ and $\Upsilon_{p q}(0) \equiv Y_{p q}(0) / \sqrt{Y_{p p}(0) Y_{q q}(0)}$ for $L=40$ and $p, q<L / 2$ (specifically, $p, q=1$ to 10) in Figure 6.2. The values of the off-diagonal elements of $\chi_{p q}(0)$ and $\Upsilon_{p q}(0)$ are not zero (we do not expect them to be zero even after caring for numerical accuracy); however, they are at least two orders of magnitude smaller than the diagonal ones. 


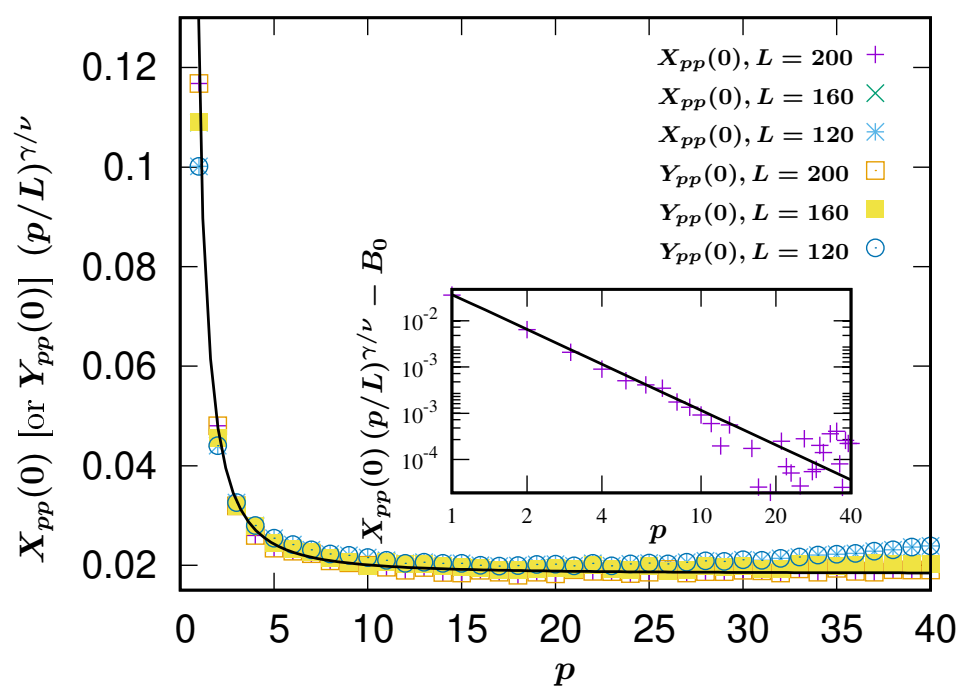

Figure 6.1: $X_{p p}(0)$ and $Y_{p p}(0)$ as a function of $p$ for different system sizes, with $p=1$ to 40 , and $L=120,160,200$. Fitting to the data leads to $X_{p p}(0)=Y_{p p}(0) \approx$ $(L / p)^{\gamma / \nu}\left(B_{0}+B_{1} p^{-1.75}\right)$, where $B_{0}=0.0185$ and $B_{1}=0.1$. Inset: $X_{p p}(0)$ data for $L=200$ is fitted in a log-log plot; the straight line has slope -1.75 .

Together these results indicate that to a very good approximation the modes remain statistically independent during the system's evolution by means of Kawasaki dynamics.

\subsubsection{Fourier modes as approximate dynamical eigenmodes of the model}

In Figure 6.3, we obtain a data collapse plot for the mean-square deviation (MSD) of the complex mode amplitude $\left\langle\left|\Delta A_{p}^{2}(t)\right|\right\rangle$, as a function of $(p / L)^{z_{c}} t$ for $p=1,2, \ldots, 10$ for three different system sizes $L=120,160,200$ (from our earlier works on spin systems $[28,74,76]$ we expect that the data collapse would require scaling time with a prefactor $\left.(p / L)^{z_{c}}\right)$. The solid line in the figure then represents

$$
\begin{array}{r}
\left\langle\Delta A_{p}^{2}(t)\right\rangle=\sqrt{2}\left\langle\Delta X_{p}^{2}(t)\right\rangle=\sqrt{2}\left\langle\Delta Y_{p}^{2}(t)\right\rangle \approx 3.2527\left(\frac{L}{p}\right)^{\gamma / \nu}(p / L)^{z_{c}} t \\
\text { for }(p / L)^{z_{c}} t \ll 1 .
\end{array}
$$

Since the MSDs of the mode amplitudes can be expressed in terms of their auto- 

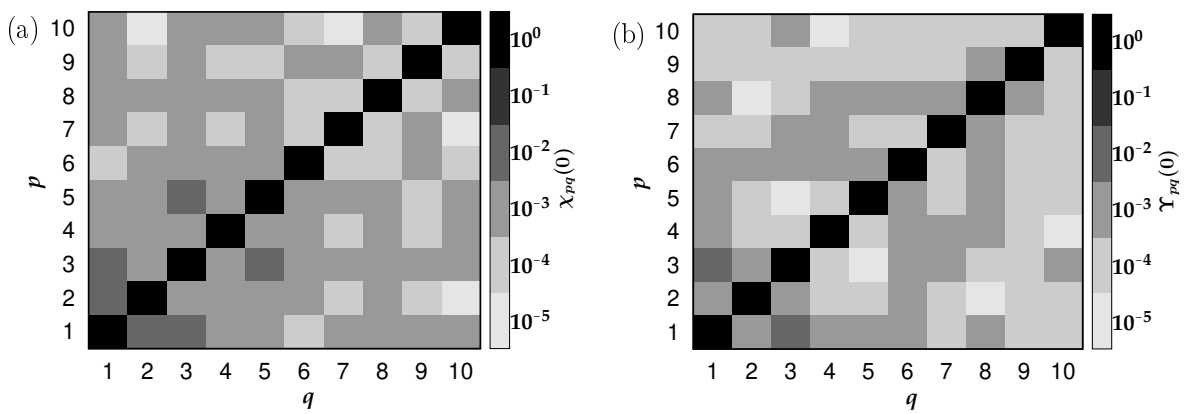

Figure 6.2: The matrix (a), $\chi_{p q}(0) \equiv X_{p q}(0) / \sqrt{X_{p p}(0) X_{q q}(0)}$ and (b), $\Upsilon_{p q}(0) \equiv$ $Y_{p q}(0) / \sqrt{Y_{p p}(0) Y_{q q}(0)}$ in logarithmic greyscale for $p, q=1,2, \ldots, 10$ and $L=40$. The values of the off-diagonal elements of $\chi_{p q}(0)$ and $\Upsilon_{p q}(0)$ are not zero. However, most of them are typically two or more orders of magnitude smaller than the diagonal ones, which means the modes are statistically uncorrelated.

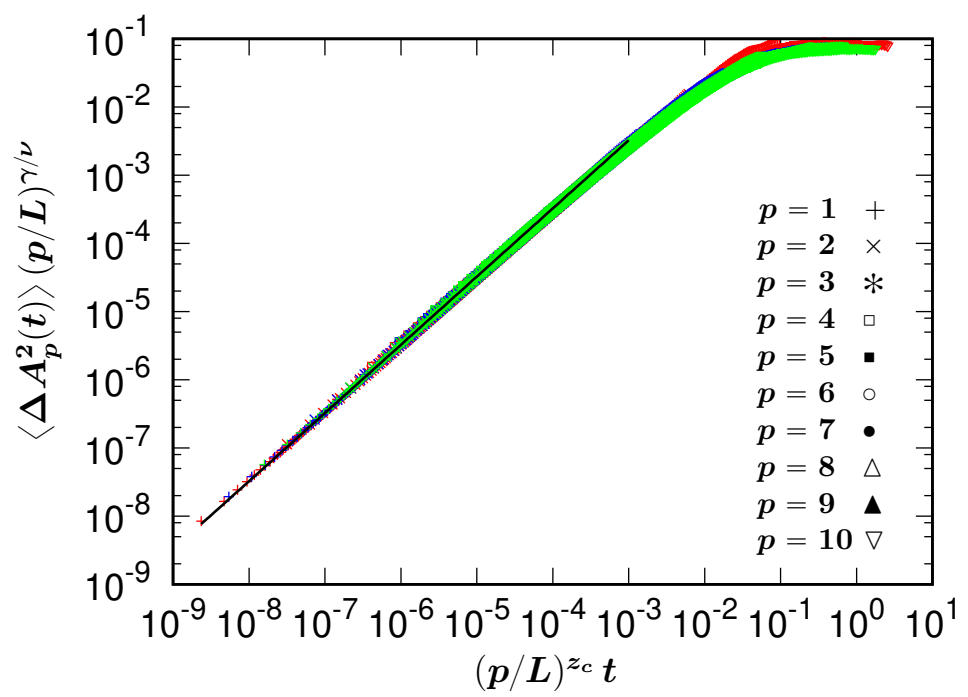

Figure 6.3: The MSD of the complex modes amplitude $\left\langle\left|\Delta A_{p}^{2}(t)\right|\right\rangle=\sqrt{2}\left\langle\Delta Y_{p}^{2}(t)\right\rangle=$ $\sqrt{2}\left\langle\Delta X_{p}^{2}(t)\right\rangle$, where $\left\langle\Delta X_{p}^{2}(t)\right\rangle=\left\langle\left[X_{p}(t)-X_{p}(0)\right]^{2}\right\rangle$ and $\left\langle\Delta Y_{p}^{2}(t)\right\rangle=\left\langle\left[Y_{p}(t)-\right.\right.$ $\left.\left.Y_{p}(0)\right]^{2}\right\rangle$. For every system size $L=120$ (red), 160 (blue), 200 (green), the MSD of ten different mode amplitudes are measured. In the range $t \lesssim(p / L)^{z_{c}}$, the modes shows normal diffusion and the solid line represents $\left\langle\left|\Delta A_{p}^{2}(t)\right|\right\rangle(p / L)^{\gamma / \nu} \approx$ $3.2527(p / L)^{z_{c}} t$. 
6.3. Dynamics of two physical observables using the Fourier modes as approximate 72

correlation functions as

$$
\begin{aligned}
\left\langle\Delta X_{p}^{2}(t)\right\rangle & =\left\langle\left[X_{p}(t)-X_{p}(0)\right]^{2}\right\rangle=2 X_{p p}(0)\left[1-\frac{X_{p p}(t)}{X_{p p}(0)}\right] \\
\left\langle\Delta Y_{p}^{2}(t)\right\rangle & =\left\langle\left[Y_{p}(t)-Y_{p}(0)\right]^{2}\right\rangle=2 Y_{p p}(0)\left[1-\frac{Y_{p p}(t)}{Y_{p p}(0)}\right]
\end{aligned}
$$

with the approximation $X_{p p}(0)=Y_{p p}(0) \approx(L / p)^{\gamma / \nu}\left(B_{0}+B_{1} p^{-1.75}\right)$, for $(p / L)^{z_{c}} t \ll$ 1 in a large range shown in Figure 6.3, Eqs. (6.10-6.11) can be recast in the form

$$
\frac{X_{p p}(t)}{X_{p p}(0)}=\frac{Y_{p p}(t)}{Y_{p p}(0)} \approx \exp \left[-\frac{1.15(p / L)^{z_{c}} t}{0.0185+0.1 p^{-\gamma / \nu}}\right] \text {. }
$$

To conclude, in this section we have demonstrated that to a very good approximation the Fourier modes for the 2D Ising model with Kawasaki dynamics remain statistically uncorrelated at all times, and their autocorrelations decay exponentially in time, from which we conclude that they are approximate dynamical eigenmodes. This means that the properties of the modes amplitude can be used to calculate all dynamical quantities to a very good approximation $[56,59,119,120]$. In the following section, we will showcase this to calculate the autocorrelation function and the MSD of line magnetization.

\subsection{Dynamics of two physical observables using the Fourier modes as approximate dynamical eigenmodes}

In this section we focus on the dynamics observables of the system. Using the properties of the Fourier modes obtained in the last section, we analytically derive the autocorrelation function and the mean-square deviation of the line magnetization.

\subsubsection{Autocorrelation function of the line magnetization}

The first dynamical observable we are dealing with is the autocorrelation function of the line magnetization, defined as

$$
C(t)=\left\langle M_{l}(x, t) M_{l}(x, 0)\right\rangle .
$$

This autocorrelation function can be expressed in terms of the modes by combining Eqs. (6.6), (6.9) and (6.13), yielding

$$
\begin{aligned}
C(t) & =4 \sum_{p=1}^{L / 2} X_{p p}(t) \\
& =4 \sum_{p=1}^{L / 2}\left(\frac{L}{p}\right)^{\gamma / \nu} \exp \left[-\frac{1.15(p / L)^{z_{c}} t}{0.0185+0.1 p^{-\gamma / \nu}}\right]\left(0.0185+0.1 p^{-\gamma / \nu}\right)
\end{aligned}
$$

As shown in Figure 6.4, the prediction (6.14) fits the simulation results quite well. 
Approximate dynamical eigenmodes of the Ising model with local spin-exchange moves

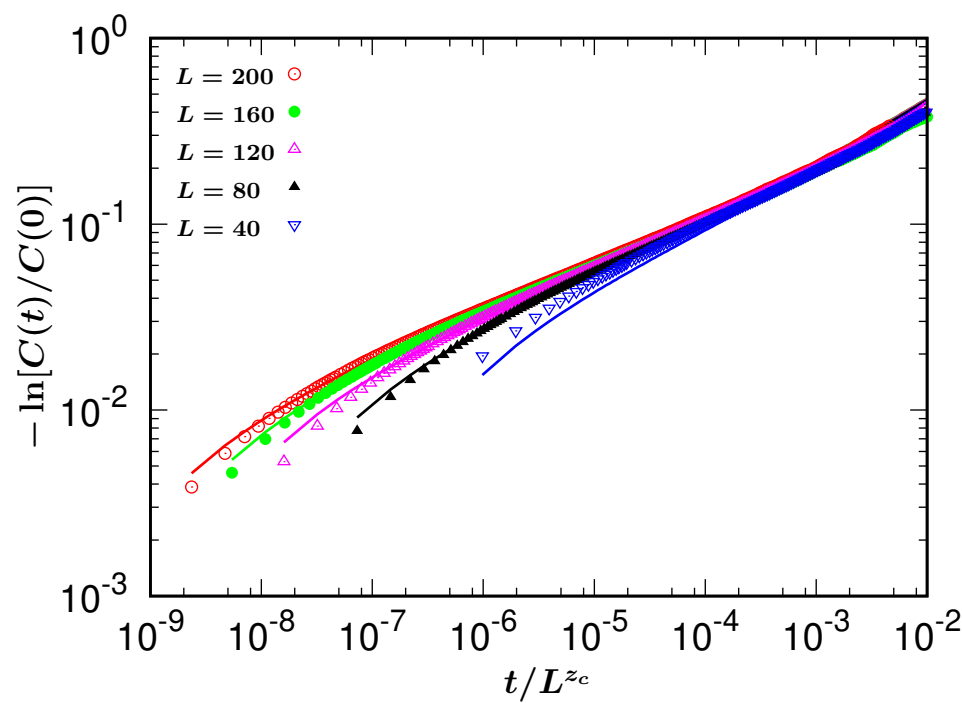

Figure 6.4: (color online) Comparison between the simulation results (points) and expectation values from Eq. (6.14) (solid lines, same colors as the points) for the autocorrelation function $C(t)$ of the line magnetization, for different system sizes.

\subsubsection{Anomalous diffusion of the line magnetization}

Let us now consider the MSD of the line magnetization

$$
\left\langle\Delta M_{l}^{2}(t)\right\rangle=\left\langle\left[M_{l}(x, t)-M_{l}(x, 0)\right]^{2}\right\rangle
$$

as another dynamical observable.

Using Eq. (6.6) and $\left\langle X_{p}(t) Y_{q}(0)\right\rangle=\left\langle Y_{p}(t) X_{q}(0)\right\rangle=0$, we have

$$
\begin{aligned}
\left\langle\Delta M_{l}^{2}(t)\right\rangle= & \sum_{p=0}^{L-1} \sum_{q=0}^{L-1}\left\langle\left[X_{p}(t)-X_{p}(0)\right]\left[X_{q}(t)-X_{q}(0)\right] \cos (2 \pi p x / L) \cos (2 \pi q x / L)\right. \\
& \left.+\left[Y_{p}(t)-Y_{p}(0)\right]\left[Y_{q}(t)-Y_{q}(0)\right] \sin (2 \pi p x / L) \sin (2 \pi q x / L)\right\rangle .
\end{aligned}
$$

Then Eq. (6.16) can be simplified with the approximation $X_{p q}(t)=Y_{p q}(t)=$ $X_{p(L-q)}(t)=Y_{p(L-q)}(t)$, and $X_{0}(t)$ as the conserved order parameter (chosen to be 
6.3. Dynamics of two physical observables using the Fourier modes as approximate 74

dynamical eigenmodes

zero) of the dynamics, leading us to

$$
\begin{aligned}
\left\langle\Delta M_{l}^{2}(t)\right\rangle & =2 \sum_{p=1}^{L-1} \sum_{q=1}^{L-1}\left[X_{p q}(0)-X_{p q}(t)\right] \\
& =8 \sum_{p=1}^{L / 2} \sum_{q=1}^{L / 2} X_{p q}(0)\left[1-\frac{X_{p q}(t)}{X_{p q}(0)}\right] \\
& =8 \sum_{p=1}^{L / 2} X_{p p}(0)\left[1-\frac{X_{p p}(t)}{X_{p p}(0)}\right] .
\end{aligned}
$$

Using the properties of $X_{p p}(t)$ and $X_{p p}(0)$ as obtained in Secs. 6.2.3-6.2.4, the behavior of the MSD of the line magnetization can be divided into two time domains.

At long times $t \gtrsim L^{z_{c}}, \frac{X_{p p}(t)}{X_{p p}(0)} \rightarrow 0$, meaning that $\left\langle\Delta M_{l}^{2}(t)\right\rangle$ approaches a constant $\sim L^{\gamma / \nu}$. At intermediate times $1 \lesssim t \lesssim L^{z_{c}}$,

$$
\begin{aligned}
\left\langle\Delta M_{l}^{2}(t)\right\rangle & =8 \sum_{p=1}^{L / 2} X_{p p}(0)\left[1-\frac{X_{p p}(t)}{X_{p p}(0)}\right] \\
& =8 \sum_{p=1}^{L / 2}\left(\frac{L}{p}\right)^{\gamma / \nu}\left[1-\exp \left(-\frac{1.15(p / L)^{z_{c}} t}{0.0185+0.1 p^{-\gamma / \nu}}\right)\right]\left(0.0185+0.1 p^{-\gamma / \nu}\right) .
\end{aligned}
$$

As shown in Figure 6.5 (a), the prediction (6.18) fits the simulation results quite well.

For an analytical expression for the MSD, with $x=p / L$, the sum (6.18) can be reduced to the following integral:

$$
\begin{array}{r}
\left\langle\Delta M_{l}^{2}(t)\right\rangle=8 L \int_{1 / L}^{1 / 2} \frac{d x}{x^{\gamma / \nu}}\left(1-\exp \left[-\frac{1.15 t x^{z_{c}}}{\left\{0.0185+0.1 /(x L)^{\gamma / \nu}\right\}}\right]\right) \\
\left\{0.0185+0.1 /(x L)^{\gamma / \nu}\right\},
\end{array}
$$

but beyond that it is difficult to process it further without making approximations. In particular, in the limit $L \rightarrow \infty$ and finite values of $x$, the second term within the curly brackets can be dropped. At the lower limit of $x$, the two terms within the curly brackets are however comparable. Nevertheless, if we do drop this second term altogether, then the integral can be easily performed to show that in the leading order of $L$

$$
\begin{aligned}
\left\langle\Delta M_{l}^{2}(t)\right\rangle & \sim L\left(\frac{t}{L}\right)^{(\gamma / \nu-1) / z_{c}} \Rightarrow \\
\left\langle\Delta M_{l}^{2}(t)\right\rangle & \sim L^{\gamma / \nu}\left(\frac{t}{L^{z_{c}}}\right)^{(\gamma / \nu-1) / z_{c}} \\
& \approx L^{\gamma / \nu}\left(\frac{t}{L^{z_{c}}}\right)^{0.2}
\end{aligned}
$$


Approximate dynamical eigenmodes of the Ising model with local spin-exchange moves

This behavior of the sum (6.20) is shown in Figure 6.5(b).
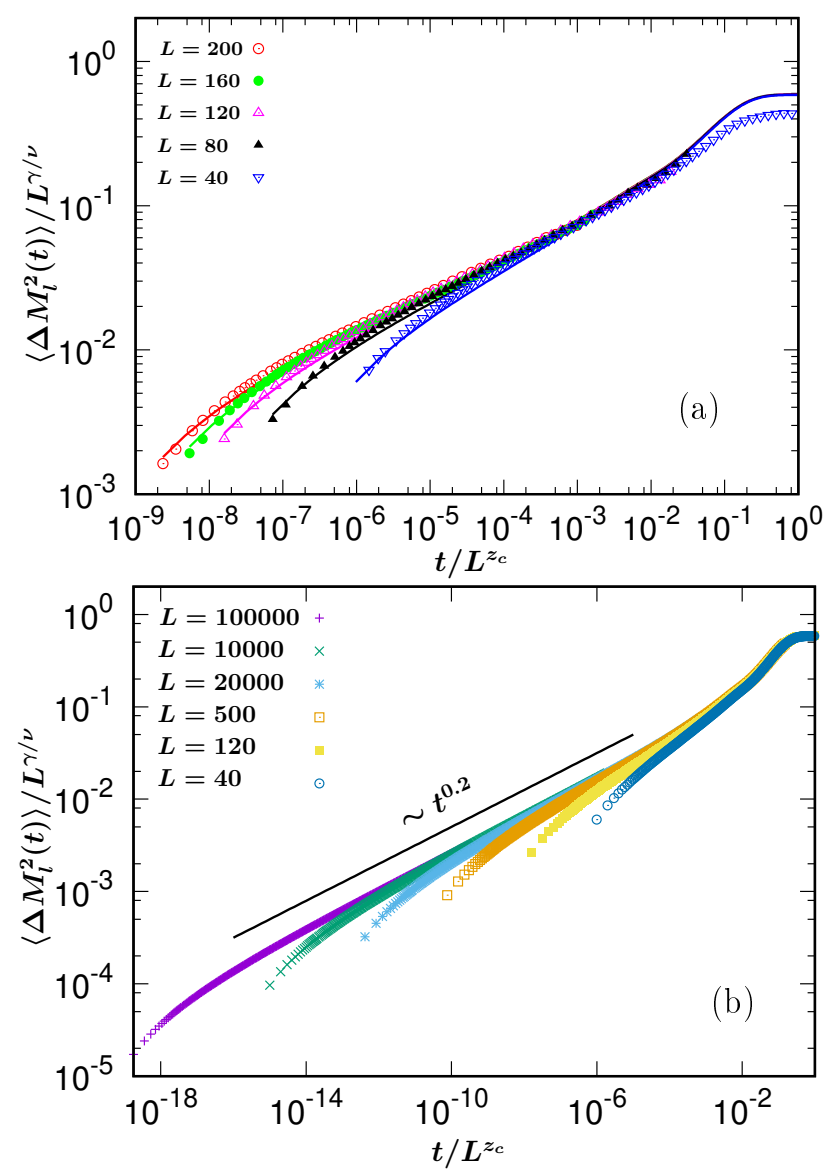

Figure 6.5: (a) Comparison between the simulation results (points) and the results obtained from Eq. (6.18) (solid lines, same color as the points) for the MSD of the line magnetization $\left\langle\Delta M_{l}^{2}(t)\right\rangle$ for different system sizes. (b) Confirmation of the sum (6.20) to power-law $t^{(\gamma / \nu-1) / z_{c}} \approx t^{0.2}$ for $L \rightarrow \infty$.

\subsection{Generalised Langevin Equation formulation for the anomalous diffusion in the Ising model with Kawasaki dynamics}

In Sec. 6.3 we have demonstrated that at the intermediate time regime, the line magnetization in the Ising model with Kawasaki dynamics exhibits anomalous diffusion. In 
6.4. Generalised Langevin Equation formulation for the anomalous diffusion in the 76

Ising model with Kawasaki dynamics

our recent studies on the Ising and $\phi^{4}$ model with Glauber dynamics [74, 76], we have argued that the anomalous diffusion of the magnetization belongs to the GLE class, for which the restoring force plays an important role.

Imagine that we choose a tagged line, and since the thermal spin flips, at $t=0$ its magnetization $M_{l}$ changes by a little amount $\delta M_{l}$. The surrounding spins will react to this change due to the interactions dictated by the Hamiltonian, and it takes time to spread this reaction. During this time, the value of $M_{l}$ will also readjust to the persisting values of the surrounding spins, undoing at least a part of $\delta M_{l}$. It is the latter that we interpret as the result of "inertia" of the surrounding spins that resists changes in $M_{l}$, and the resistance itself acts as the restoring force to the changes in the tagged magnetization, and finally, leads to anomalous diffusion.

\subsubsection{Generalized Langevin Equation for the line magnetization}

From how the restoring force works introduced before, it not only indicates that there is a memory effect which is significant during the 'restoring' process, but also leads us to the GLE formulation to describe the anomalous diffusion.

In line with our previous works on the Ising and $\phi^{4}$ model with Glauber dynamics $[74,76]$ and in polymeric systems $[53,54,55,59]$, the relation of the restoring force $f(t)$ and the "velocity" of magnetization $\dot{M}_{l}(t)$ can be expressed as

$$
\begin{gathered}
\zeta \dot{M}_{l}(t)=f(t)+q_{1}(t) \\
f(t)=-\int_{0}^{t} d t^{\prime} \mu\left(t-t^{\prime}\right) \dot{M}_{l}\left(t^{\prime}\right)+q_{2}(t) .
\end{gathered}
$$

Here $f(t)$ is the internal force, $\zeta$ is the "viscous drag" on $M_{l}, \mu\left(t-t^{\prime}\right)$ is the memory kernel, $q_{1}(t)$ and $q_{2}(t)$ are two noise terms satisfying $\left\langle q_{1}(t)\right\rangle=\left\langle q_{2}(t)\right\rangle=0$, and the fluctuation-dissipation theorems (FDTs) are given by $\left\langle q_{1}(t) q_{1}\left(t^{\prime}\right)\right\rangle \propto \zeta \delta\left(t-t^{\prime}\right)$ and $\left\langle q_{2}(t) q_{2}\left(t^{\prime}\right)\right\rangle \propto \mu\left(t-t^{\prime}\right)$ respectively.

Equation (6.21b) can be inverted to write as

$$
\dot{M}_{l}(t)=-\int_{0}^{t} d t^{\prime} a\left(t-t^{\prime}\right) f\left(t^{\prime}\right)+\omega(t) .
$$

The noise term $\omega(t)$ similarly satisfies $\langle\omega(t)\rangle=0$, and the FDT $\left\langle\omega(t) \omega\left(t^{\prime}\right)\right\rangle=a(\mid t-$ $\left.t^{\prime} \mid\right)$. Then $a(t)$ and $\mu(t)$ are related to each other in the Laplace space as $\tilde{a}(s) \tilde{\mu}(s)=1$.

To combine Eq. (6.21a) and (6.21b), we obtain

$$
\zeta \dot{M}_{l}(t)=-\int_{0}^{t} d t^{\prime} \mu\left(t-t^{\prime}\right) \dot{M}_{l}\left(t^{\prime}\right)+q_{1}(t)+q_{2}(t) .
$$

or

$$
\dot{M}_{l}(t)=-\int_{0}^{t} d t^{\prime} \theta\left(t-t^{\prime}\right)\left[q_{1}(t)+q_{2}(t)\right] .
$$

where in the Laplace space $\tilde{\theta}(s)[\zeta+\tilde{\mu}(s)]=1$. With $t>t^{\prime}$, without any loss of generality, using Eq. (6.24) the result of the velocity autocorrelation is

$$
\left\langle\dot{M}_{l}(t) \dot{M}_{l}(0)\right\rangle \sim \theta\left(t-t^{\prime}\right),
$$


Approximate dynamical eigenmodes of the Ising model with local spin-exchange moves

where $\theta(t)$ can be calculated by Laplace inverting the relation $\tilde{\theta}(s)[\zeta+\tilde{\mu}(s)]=1$.

If the memory term is a power law in time, i.e.,

$$
\mu(t) \sim t^{-c}
$$

Using the results from Ref. [54], we have

$$
\left.\left\langle\dot{M}_{l}(t) \dot{M}_{l}(0)\right\rangle\right|_{f=0} \sim-\left(t-t^{\prime}\right)^{c-2} .
$$

By integrating Eq. (6.27) twice in time, we obtain that

$$
\left\langle\Delta M_{l}^{2}(t)\right\rangle \sim t^{c}
$$

In summary, there is a power-law memory function $\mu(t) \sim t^{-c}$ which plays a vital part in the GLE formulation. From this we can deduce that the anomalous diffusion found in Eq. (6.18) is non-Markovian and the anomalous exponent is $c$.

\subsubsection{Verification of the power-law behavior of $\mu(t)$}

Based on the FDT mentioned under Eq. (6.21b), we now numerically verify the behavior of $\mu(t)$.

During simulations, at $t=0$, we thermalise the system to its equilibrium state. For $t>0$ we select a line and fix its value of the magnetization $M_{l}$ by performing non-local spin-exchange dynamics, i.e., we choose two lattice sites $(j, k)$ and $(m, n)$, if $s_{j, k} s_{m, n}=-1$ then we exchange their values, else we keep their values as they are. The energy change $\Delta E^{\prime}$ is measured and we accept the move with the Metropolis probability $\min \left(1, \exp \left(-\Delta E^{\prime} / T\right)\right)$. For the rest of the system, we let them evolve with the Kawasaki dynamics.

We then keep taking snapshots of the system at regular intervals. For every snapshot we take, we consider an attempt to flip each spin in turn and find the expected change in $M_{l}$ which would have occurred if this move had been implemented, totaled over all the spins on the selected line, and the possible change of the line magnetization is defined as $f(t)=\dot{M}(t)$. The quantity $\langle f(t) f(0)\rangle$ is plotted in Figure 6.6. The figure is in good agreement with our expectation that $\mu(t) \sim t^{-(\gamma / \nu-1) / z_{c}}$; this result has also been observed for the the 2D Ising model with Glauber dynamics [74].

\subsection{Conclusion}

In this chapter, we have studied the Fourier modes of the two-dimensional Ising model with Kawasaki dynamics at critical temperature and at zero (conserved) order parameter. We have established that the Fourier modes are the dynamical eigenmodes of the system to a very good approximation. Using these modes, we can reconstruct the dynamics of any dynamical variable; we have done so for the autocorrelation function and the mean-square deviation (MSD) of line magnetization.

At the intermediate times, we have found that for $1 \lesssim t \lesssim L^{z_{c}}$, the line magnetization undergoes anomalous diffusion. We have argued that like other spin models and 


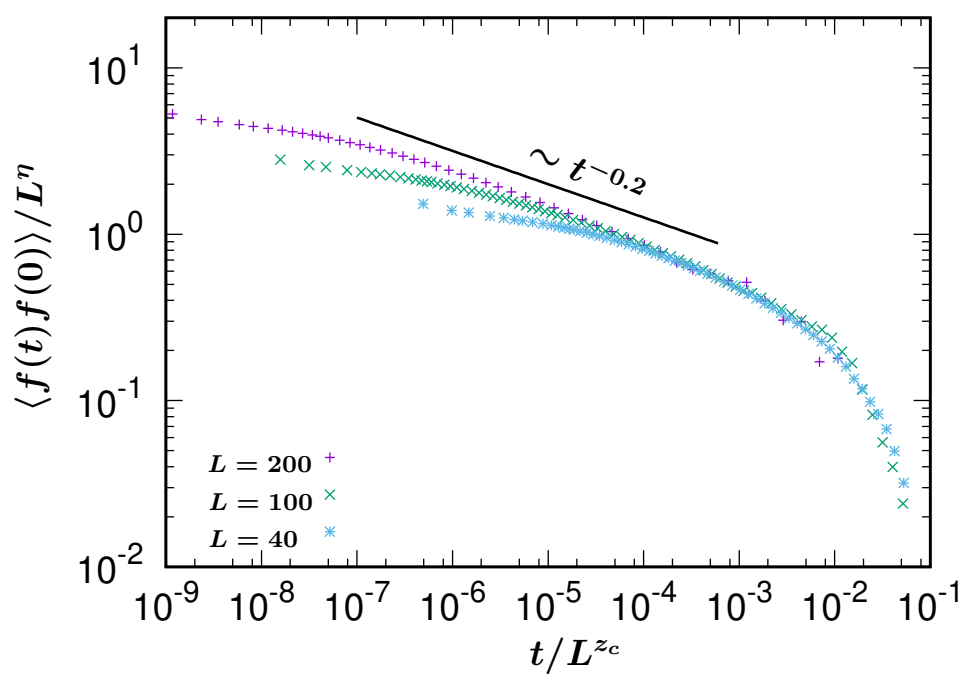

Figure 6.6: The autocorrelation function $\langle f(t) f(0)\rangle$ as a function of time; the solid line corresponds to $\langle f(t) f(0)\rangle \sim t^{-(\gamma / \nu-1) / z_{c}} \approx t^{-0.2}$.

polymeric systems this anomalous behavior can be described by the GLE formulation with a memory kernel. The corresponding fluctuation-dissipation theorem has been verified by the calculation of the force autocorrelation.

With these results, we have showcased that for Kawasaki dynamics, the Fourier modes, as the approximate dynamical eigenmodes, is a useful tool to analytically derive the dynamical quantities in the Ising system. We however note that if the model is evolved using Glauber dynamics, then we find that $X_{p p}(t)$ decays as a stretched exponential in time (not shown in this chapter), which clearly shows that the Fourier modes are not the (approximate) dynamical eigenmodes. We do not understand this at present. It could be explored in the future.

\subsection{Appendix: Scaling of $\left\langle\left|A_{p}\right|\right\rangle^{2}$ with $p$ for the 2D Ising model}

In this appendix we obtain the scaling behavior of $\left\langle\left|A_{p}\right|^{2}\right\rangle$ for the 2D Ising model (note that the calculations presented here do not correspond to the total magnetization of the sample kept fixed at zero, as is the case for Kawasaki dynamics in this chapter).

First we calculate the autocorrelation function of the line magnetization. We use the classic result that at the critical temperature the spin-spin autocorrelation function decays as $r^{-\eta}$, where $r$ is the Euclidean distance between the two spins and $\eta=$ $2-\gamma / \nu=0.25$ for the 2D Ising model. With that knowledge, upon summing over $i$ 
Approximate dynamical eigenmodes of the Ising model with local spin-exchange moves

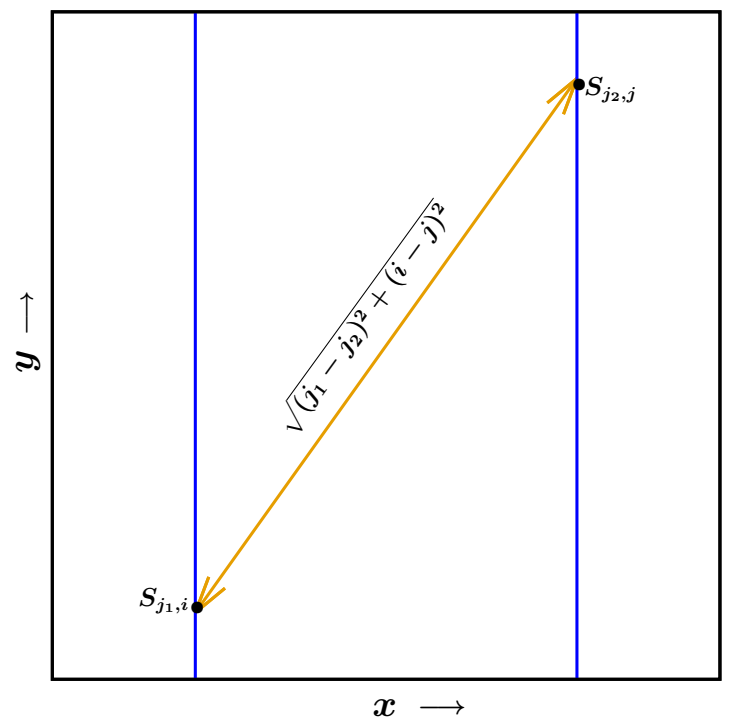

Figure 6.7: Schematic diagram for the calculation of the line-line autocorrelation function.

and $j$ in the $y$-direction (see Figure 6.7), we obtain

$$
\left\langle M_{l}\left(j_{1}, 0\right) M_{l}\left(j_{2}, 0\right)\right\rangle=\sum_{i=0}^{L-1} \sum_{j=0}^{L-1}\left\langle s_{j_{1}, i} s_{j_{2}, j}\right\rangle \sim \sum_{i=0}^{L-1} \sum_{j=0}^{L-1}\left[(i-j)^{2}+\left(j_{2}-j_{1}\right)^{2}\right]^{-\eta / 2} \text {. }
$$

We next set $a=\left(j_{1}-j_{2}\right) / L, u=(i-j) / L$ and $v=j / L$ to write order,

$$
\left\langle M_{l}\left(j_{1}, 0\right) M_{l}\left(j_{2}, 0\right)\right\rangle \sim \int_{-1}^{1} d u \frac{L^{2-\eta}}{\left[u^{2}+4 a^{2}\right]^{\eta / 2}} .
$$

The calculation of $\left\langle\left|A_{p}\right|^{2}\right\rangle$ follows from Eq. (6.30) in a similar manner.

$$
\left\langle\left|A_{p}\right|^{2}\right\rangle=\frac{1}{L^{2}} \sum_{j_{1}=0}^{L} \sum_{j_{2}=0}^{L}\left\langle M_{l}\left(j_{1}, 0\right) M_{l}\left(j_{2}, 0\right)\right\rangle \cos \left[2 \pi p\left(j_{1}-j_{2}\right) / L\right] .
$$

This time setting $a \rightarrow a / 2$, Eq. (6.31) reduces to

$$
\begin{aligned}
\left\langle\left|A_{p}\right|^{2}\right\rangle & \sim L^{2-\eta} \int_{-1}^{1} d a \int_{-1}^{1} d u \frac{1}{\left[u^{2}+a^{2}\right]^{\eta / 2}} \cos (\pi p a) \\
& =4 L^{2-\eta} \int_{0}^{1} d a \int_{0}^{1} d u \frac{1}{\left[u^{2}+a^{2}\right]^{\eta / 2}} \cos (\pi p a) .
\end{aligned}
$$

For $p=0$, Eq. (6.32) leads to $\left|A_{p}(0)\right|^{2} \sim L^{2-\eta}$, which is the classic result for the equilibrium scaling $\left\langle M^{2}\right\rangle \sim L^{4-\eta}=L^{2+\gamma / \nu}$ for the total sample magnetization $M$ for the 2D Ising model. 
For $p \neq 0$ we perform the integration over $u$ in Eq. (6.32) to obtain

$$
\left\langle\left|A_{p}\right|^{2}\right\rangle \sim L^{2-\eta} \underbrace{\int_{0}^{1} d a f(a) \cos (\pi p a)}_{I(p)},
$$

with

$$
\begin{aligned}
f(a)= & \frac{\left(1+a^{2}\right)^{1-\eta / 2}\left(5+a^{2}-\eta\right)}{(4-\eta)(2-\eta)} \\
& -\frac{\left(1+a^{2}\right)^{3-\eta / 2} \text { Hypergeometric }{ }_{2} F_{1}\left(1,(3-\eta) / 2,-1 / 2,-1 / a^{2}\right)}{a^{2}(4-\eta)(2-\eta)} .
\end{aligned}
$$
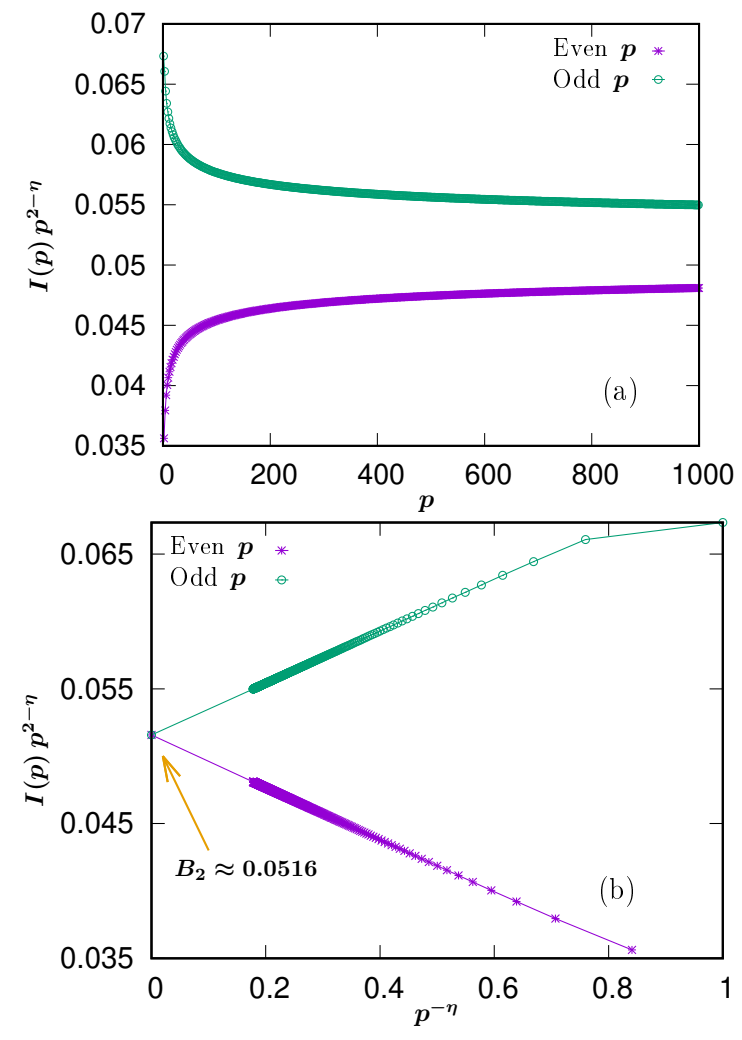

Figure 6.8: (a) Numerical integration of the integral in Eq. (6.34). (b) The figure shows that $I(p) \approx B_{2} p^{2-\eta}$ in the limit $p \rightarrow \infty$, where $B_{2} \approx 0.0516$, although convergence to the asymptotic behavior is rather slow. The solid lines are fits to the data.

We then perform numerical integration separately for even and odd $p$-values for 
Approximate dynamical eigenmodes of the Ising model with local spin-exchange moves

Eq. (6.34). The results, shown in Figure 6.8, demonstrate that in the limit $p \rightarrow \infty$

$$
\left\langle\left|A_{p}\right|\right\rangle^{2} \sim\left(\frac{L}{p}\right)^{2-\eta}=\left(\frac{L}{p}\right)^{\gamma / \nu},
$$

although convergence to the asymptotic behavior is rather slow. 

CHAPTER 7

\section{Conclusion and outlook}

Although anomalous diffusion is a ubiquitous phenomenon in physics, biology, and many other research fields, it is only recently that the phenomenon was observed in the $2 D$ Ising model at its critical temperature [28]. In this thesis, we have found that anomalous diffusion is quite a common phenomenon in Ising-like models at their critical point. The models considered in this thesis are the normal Ising model, the $2 D$ scalar $\phi^{4}$ model, and the bond-diluted Ising model.

In chapter 2 , the anomalous diffusion is found as a common phenomenon at the critical point for both $2 D$ and $3 D$ Ising models. By calculating the velocity autocorrelation function of the magnetization and the autocorrelation function of the restoring force, we have numerically proven that the GLE is a proper model to describe the anomalous diffusion in the Ising model. Besides, we find that the anomalous exponent can be used to measure the dynamical exponent for Ising-like systems.

We extend our findings to the off-critical temperatures in the Ising model in chapter 3. The anomalous exponent flow, i.e., $\dot{c}$ as a function of $c$, is measured, where a true exponent is defined as $\dot{c} \equiv 0$. As we have obtained that at a temperature larger than $T_{c}$, there is a true exponent for each temperature, while for $T<T_{c}$, there is no true exponent but only a peak value for each temperature. Around the critical temperature, both the true exponents and the peak values are flowing away from the critical anomalous exponent, providing us a new insight to understand the phase transition.

Further, we claim that the anomalous exponent can be treated as a method to measure the dynamical exponent. We test the idea in the $2 D$ scalar $\phi^{4}$ model (chapter 4) and the $2 D$ bond-diluted Ising model (chapter 5). In the $2 D$ scalar $\phi^{4}$ model, the obtained dynamical exponent confirms that it belongs to the $2 D$ Ising universality class. In the $2 D$ bond-diluted Ising model, we find that when the bond concentration ap- 
proaches the percolation threshold, the dynamical exponent is increasing to infinity. It means that the system is getting slower and slower. This phenomenon is regarded as the "super slowing down" behavior.

After numerically proving and measuring different kinds of dynamical properties, we turn our attention to find a way to calculate those dynamical properties analytically. The $2 D$ Ising model with Kawasaki dynamics is studied in chapter 6 . We find that the Fourier modes of the total magnetization are good approximations to be the dynamical eigenmodes. The MSD and the autocorrelation function of the magnetization are derived with the approximate dynamical eigenmodes. The analytical results fit the numerical ones quite well. It also reduces the impact of the finite-size effects, i.e., we can have results for vary large system sizes with the analytical method, which provides us more accurate estimations of the anomalous exponent and other dynamical properties.

Until now, we have studied the anomalous diffusion in several Ising-like models. However, there are many unexplored areas in the field of combining anomalous diffusion and phase transitions.

In the future, we would like to figure out whether anomalous diffusion of the order parameter can be found at the critical point in other systems with a second-order phase transition. For particular interests, we want to extend our findings to the glassy states. There are many unknowns in the systems undergoing glass transition, we hope that the dynamical properties like the mean-square displacement of the order parameters can provide some useful information that will deepen our understanding of glass transition.

Finally, as a fantastic field, active matter catches a lot of attention in the last few years [135]. One of the famous ideal models is the active Brownian particles (ABPs) [136], which is a model with a gas-fluid like transition diagram. However, at the critical point, it hard to obtain accurate equilibrium properties, even the precise position of the critical point is not yet clear. Besides, it is still under debate whether the critical behavior at the critical point of the ABPs belongs to the Ising universality class or not $[137,138]$. We believe that the dynamical quantities will provide more convincing evidence to solve this debate. The idea will be tested later. 
[1] R. Klages, G. Radons, I. M. Sokolov (Eds.), Anomalous transport, Wiley-VCH, Weinheim (2008).

[2] J.-P. Bouchaud and A. Georges, Anomalous diffusion in disordered media: statistical mechanisms, models and physical applications. Phy. Rep. 195127 (1990).

[3] S. Havlin, D. Ben-Avraham, Diffusion in disordered media. Adv. Phys. 51, 187 (2002).

[4] R. N. Mantegna and H. E. Stanley, An Introduction to Econophysics; Correlations and Complexity in Finance, Cambridge University Press, Cambridge (2000).

[5] F. Höfling, T. Franosch, Anomalous transport in the crowded world of biological cells. Rep. Prog. Phys. 76, 046602 (2013).

[6] P. Dieterich, R. Klages, R. Preuss and A. Schwab, Anomalous dynamics of cell migration. Proc. Natl. Acad. Sci. 105, 459 (2007).

[7] G. Ariel et al., Swarming bacteria migrate by Lévy Walk. Nature Comm. 6, 8396 (2015).

[8] G. M. Viswanathan, M. G. E. da Luz, E. P. Raposo and H. E. Stanley, The Physics of Foraging, Cambridge University Press, Cambridge (2011).

[9] P. G. de Gennes, La percolation: un concept unificateur (Percolation a unifying concept). La Recherche 7, 919 (1976).

[10] Y. Gefen and A. Aharony, Anomalous diffusion on percolating clusters. Phys. Rev. Lett. 50, 77 (1983).

[11] F. Höfling, T. Franosch, E. Frey, Localization transition of the three-dimensional Lorentz model and continuum percolation. Phys. Rev. Lett. 96, 165901 (2006). 
[12] H. Scher and E. W. Montroll, Anomalous transit-time dispersion in amorphous solids. Phys. Rev. B 122455 (1975).

[13] J. Klafter, A. Blumen and M. F. Shlesinger, Stochastic pathway to anomalous diffusion. Phys. Rev. A 35, 3081 (1987).

[14] J. Klafter, M. F. Shlesinger and G. Zumofen, "Beyond brownian motion", Physics Today 49, 33 (1996); M. F. Shlesinger, J. Klafter and G. Zumofen, Above, below and beyond Brownian motion. Am. J. Phys. 67, 1253 (1999).

[15] R. Metzler and J. Klafter,The random walk's guide to anomalous diffusion: a fractional dynamics approach. Phys. Rep. 339, 1 (2000).

[16] A. N. Kolmogorov, The Wiener spiral and some other interesting curves in Hilbert space. Dokl. Acad. Sci. USSR 26, 115 (1940).

[17] B. B. Mandelbrot and J. W. van Ness, Fractional Brownian motions, fractional noises and applications. SIAM Rev. 1, 422 (1968).

[18] I. M. Tolić-Nørrelykke et al., Anomalous diffusion in living yeast cells. Phys. Rev. Lett. 93, 078102 (2004).

[19] C. Selhuber-Unkel, P. Yde, K. Berg-Sørensen, and L. B. Oddershede, Variety in intracellular diffusion during the cell cycle. Phys. Biol. 6, 025015 (2009).

[20] V. Tejedor et al, Quantitative analysis of single particle trajectories: mean maximal excursion method. Biophys. J. 98, 1364 (2010).

[21] J.-H. Jeon et al., In Vivo Anomalous Diffusion and Weak Ergodicity Breaking of Lipid Granules. Phys. Rev. Lett. 106, 048103 (2011).

[22] I. Golding and E. C. Cox, Physical nature of bacterial cytoplasm. Phys. Rev. Lett. 96, 098102 (2006).

[23] S. C. Weber, A. J. Spakowitz, and J. A. Theriot, Subdiffusive motion of a polymer composed of subdiffusive monomers. Phys. Rev. E 82, 011913 (2010).

[24] S. R. McGuffee and A. H. Elcock, Diffusion, crowding \& protein stability in a dynamic molecular model of the bacterial cytoplasm. PLoS Comput Biol. 6, e1000694 (2010).

[25] M. J. Saxton, Wanted: a positive control for anomalous subdiffusion. Biophys. J. 103, 2411 (2012).

[26] S. C. Weber, J. A. Theriot, and A. J. Spakowitz, Bacterial Chromosomal Loci Move Subdiffusively through a Viscoelastic Cytoplasm. Phys. Rev. Lett. 104, 238102 (2010). 
[27] R. Metzler, J.-H. Jeon, A. G. Cherstvya and E. Barkai, Anomalous diffusion models and their properties: non-stationarity, non-ergodicity, and ageing at the centenary of single particle tracking. Phys. Chem. Chem. Phys. 16, 24128 (2014).

[28] J. -C. Walter and G. T. Barkema, An introduction to Monte Carlo methods. Physica. A, 418, 78 (2015).

[29] M. P. Nightingale and H. W. J. Blöte, Dynamic exponent of the two-dimensional Ising model and Monte Carlo computation of the subdominant eigenvalue of the stochastic matrix. Phys. Rev. Lett. 76, 4548 (1996).

[30] E. Ising, Beitrag zur Theorie des Ferromagnetismus. Zeitschrift für Physik 31, 253 (1925).

[31] L. Onsager, Crystal statistics. I. A two-dimensional model with an order-disorder transition. Physical Review 65, 117 (1944).

[32] J. J. Binney, N. J. Dowrick, A. J. Fisher, M. E. Newman, The Theory of Critical Phenomena, Clarendon Press, Oxford (1995).

[33] H. Kleinert, V. Schulte-Frohlinde, Critical Properties of $\phi^{4}$ Theories, World Scientific, Singapore (2001)

[34] J. Kaupužs, A critical view on the perturbative RG method. Int. J. Mod. Phys. A 271250114 (2012).

[35] A. Pelissetto, E.Vicari, Critical phenomena and renormalization-group theory. Phys. Rep. 368, 549 (2002).

[36] D. J. Amit, Field Theory, the Renormalization Group, and Critical Phenomena, World Scientific, Singapore (1984).

[37] J. Zinn-Justin, Quantum Field Theory and Critical Phenomena, Clarendon Press, New York (1996).

[38] Y. Lin and F. Wang, Linear relaxation in large two-dimensional Ising models. Phys. Rev. E 93, 022113 (2016).

[39] A. Michev, D. W. Heermann, and K. Binder, Finite-size scaling analysis of the $\phi^{4}$ field theory on the square lattice. J. Stat. Phys. 44, 749 (1986).

[40] M. Hasenbusch, A Monte Carlo study of leading order scaling corrections of $\phi^{4}$ theory on a three-dimensional lattice. J. Phys. A 32, 4851 (1999).

[41] J. Kaupužs, R. V. N. Melnik and J. Rimšāns, Corrections to finite-size scaling in the $\phi^{4}$ model on square lattices. Int. J. Mod. Phys. C 27, 1650108 (2016).

[42] B. Mehling, B. M. Forrest, Universality in the critical two-dimensional $\phi^{4}$ model. Z. Phys. B 89, 89 (1992). 
[43] H. Nishimori, Transition point of the random Ising model with bond dilution. J. Phys. C: Solid State Phys. 12, L641 (1979).

[44] I. A. Hadjiagapiou, Monte Carlo analysis of the critical properties of the twodimensional randomly bond-diluted Ising model via Wang-Landau algorithm. Physica A 390, 1279 (2011).

[45] M. E. J. Newman and G. T. Barkema, Monte Carlo Methods in Statistical Physics, Oxford University Press, Oxford, (1999).

[46] N. Metropolis, et al. Equation of state calculations by fast computing machines. The Journal of Chemical Physics 21, 1087 (1953).

[47] W. K. Hastings, Monte Carlo sampling methods using Markov chains and their applications. Biometrika 57, 97 (1970).

[48] U. Wolff, Collective Monte Carlo updating for spin systems. Phys. Rev. Lett. 62, 361 (1989).

[49] R. H. Swendsen, and J. S. Wang. Nonuniversal critical dynamics in Monte Carlo simulations. Phys. Rev. Lett. 58, 86 (1987).

[50] K. Kawasaki, Diffusion constants near the critical point for time-dependent Ising models. I. Phys. Rev 145, 224 (1966).

[51] K. Binder and D. W. Heermann, Monte Carlo simulation in statistical physics: An introduction, Springer-Verlag, New York (1992).

[52] D. P. Landau and K. Binder, A guide to Monte Carlo simulations in statistical physics, Cambridge university press, Cambridge (2014).

[53] D. Panja, Generalized Langevin equation formulation for anomalous polymer dynamics. J. Stat. Mech. (JSTAT) L02001 (2010).

[54] D. Panja, Anomalous polymer dynamics is non-Markovian: memory effects and the generalized Langevin equation formulation. J. Stat. Mech. (JSTAT) P06011 (2010).

[55] D. Panja, Probabilistic phase space trajectory description for anomalous polymer dynamics. J. Phys.: Condens. Matter 23, 105103 (2011).

[56] D. Panja and G. T. Barkema, Rouse modes of self-avoiding flexible polymers. J. Chem. Phys. 131, 154903 (2009).

[57] C. Maes and S. R.Thomas, From Langevin to generalized Langevin equations for the nonequilibrium Rouse model. Phys. Rev. E 87, 022145 (2013).

[58] J. T. Bullerjahn, S. Sturm, L. Wolff and K. Kroy, Monomer dynamics of a wormlike chain. Europhys. Lett. 96, 48005 (2011). 
[59] R. Keesman, G. T. Barkema and D. Panja, Dynamical eigenmodes of star and tadpole polymers. J. Stat. Mech. (JSTAT) P02021 (2013); Dynamical eigenmodes of a polymerized membrane, ibid P04009 (2013).

[60] H. Popova and A. Milchev, Anomalous diffusion of a tethered membrane: A Monte Carlo investigation. Phys. Rev. E 77, 041906 (2008).

[61] K. Mizuochi, H. Nakanishi and T. Sakaue, Dynamical scaling of polymerized membranes. Europhys. Lett. 107, 38003 (2014).

[62] D. Panja, G. T. Barkema and R. C. Ball, Anomalous dynamics of unbiased polymer translocation through a narrow pore. J. Phys.: Condens. Mattter 19, 432202 (2007).

[63] D. Panja, G. T. Barkema and R. C. Ball, Polymer translocation out of planar confinements. J. Phys.: Condens. Matter 20, 075101 (2008).

[64] D. Panja and G. T. Barkema, Passage times for polymer translocation pulled through a narrow pore. Biophys. J. 94, 1630 (2008).

[65] J. L. A. Dubbeldam, V. G. Rostiashvili, A. Milchev and T. A. Vilgis, Fractional Brownian motion approach to polymer translocation: The governing equation of motion. Phys. Rev. E 83, 011802 (2011).

[66] H. Vocks, D. Panja, G. T. Barkema and R. C. Ball, Pore-blockade times for fielddriven polymer translocation. J. Phys.: Condens. Matter 20, 095224 (2008).

[67] D. Panja, G. T. Barkema, A. B. Kolomeisky, Through the eye of the needle: recent advances in understanding biopolymer translocation. J. Phys.: Condens. Matter 21, 242101 (2009).

[68] T. Saito and T. Sakaue, Driven anomalous diffusion: An example from polymer stretching. Phys. Rev. E 92, 012601 (2015).

[69] S. Wansleben and D. P. Landau, "Dynamical critical exponent of the 3D Ising model", J. Appl. Phys. 61, 3968 (1987).

[70] M. L. Goldstein, S. A. Morris and G. G. Yen, Problems with fitting to the powerlaw distribution. Eur. Phys. J. B 41, 255 (2004).

[71] M. Khoury, A. M. Lacasta, J. M. Sancho, and K. Lindenberg, Weak disorder: anomalous transport and diffusion are normal yet again. Phys. Rev. Lett., 106, 090602 (2011).

[72] M. Javanainen et al., Anomalous and normal diffusion of proteins and lipids in crowded lipid membranes. Faraday discussions 161, 397 (2013).

[73] R. Metzler, J. H. Jeon, and A. G. Cherstvy, Non-Brownian diffusion in lipid membranes: Experiments and simulations. Biochimica et Biophysica Acta (BBA)-Biomembranes 1858, 2451(2016). 
[74] W. Zhong, D. Panja, G. T. Barkema, and R. C. Ball, Generalised Langevin Equation Formulation for Anomalous Diffusion in the Ising Model at the Critical Temperature. Phys. Rev. E 98, 012124 (2018).

[75] W. Zhong, D. Panja and G. T. Barkema, Approximate dynamical eigenmodes of the Ising model with local spin-exchange moves. Phys. Rev. E 100, 012132 (2019).

[76] W. Zhong, G. T. Barkema, D. Panja, and R. C. Ball, Critical dynamical exponent of the two-dimensional scalar $\phi^{4}$ model with local moves. Phys. Rev. E 98, 062128 (2018).

[77] H. W. J. Blöte, M. P. Nightingale, Universal Ising dynamics in two dimensions. Physica A 251, 211 (1998).

[78] H. W. J. Blöte, E. Luijten, and J. R. Heringa, Ising universality in three dimensions: a Monte Carlo study. J. Phys. A 28, 6289 (1995).

[79] M. Hasenbusch, K. Pinn, and S. Vinti, Critical exponents of the threedimensional Ising universality class from finite-size scaling with standard and improved actions. Phys. Rev. B 59, 11471 (1999).

[80] P. C. Hohenberg and B. I. Halperin, Theory of dynamic critical phenomena. Rev. Mod. Phys. 49, 435 (1977).

[81] K. H. Hoffmann, M. Schreiber (eds), Computational Physics, Springer, Berlin,(1996).

[82] M. Y. Nalimov, V. A. Sergeev, and L. Sladkoff, Borel resummation of the $\epsilon-$ expansion of the dynamical exponent $\mathrm{z}$ in model a of the $\phi^{4}(\mathrm{O}(\mathrm{n}))$ theory. Theor. Math. Phys. 159, 499 (2009).

[83] R. C. Brower, P. Tamayo, Embedded dynamics for $\phi^{4}$ theory. Phys. Rev. Lett. 62, 1087 (1989).

[84] P. Bosetti, B. De Palma, and M. Guagnelli, Monte Carlo determination of the critical coupling in $\phi_{2}^{4}$ theory. Phys. Rev. D 92, 034509 (2015).

[85] H. E. Stanley, Phase transitions and critical phenomena, Clarendon Press, Oxford (1971).

[86] P. C. Hohenberg and B. I. Halperin, Theory of dynamic critical phenomena. Rev. Mod. Phys. 49, 435 (1977).

[87] H. G. Ballesteros et al., Ising exponents in the two-dimensional site-diluted Ising model. J. Phys. A 30, 8379 (1997).

[88] H. G. Ballesteros et al., Critical exponents of the three-dimensional diluted Ising model. Phys. Rev. B 58, 2740 (1998). 
[89] P. H. L. Martins and J. A. Plascak, Universality class of the two-dimensional site-diluted Ising model. Phys. Rev. E 76, 012102 (2007).

[90] J.-K. Kim and A. Patrascioiu, Critical behavior of the specific heat in the two dimensional site diluted Ising system. Phys. Rev. Lett. 72, 2785 (1994).

[91] R. Kenna and J. J. Ruiz-Lorenzo, Scaling analysis of the site-diluted Ising model in two dimensions. Phys. Rev. E 78, 031134 (2008).

[92] B. N. Shalaev, Critical behavior of the two-dimensional Ising model with random bonds. Phys. Rep. 237, 129 (1994).

[93] C. Jayaprakash, Eberhard K. Riedel, and Michael Wortis, Critical and thermodynamic properties of the randomly diluted Ising model. Phys. Rev. B 18, 2244 (1978).

[94] Y. L. Loh and E. W. Carlson, Efficient algorithm for random-bond Ising models in 2d. Phys. Rev. Lett. 97, 227205 (2006).

[95] M. Hasenbusch, F. P. Toldin, A. Pelissetto, and E. Vicari, Universal dependence on disorder of two-dimensional randomly diluted and random-bond $\pm J$ Ising models. Phys. Rev. E 78, 011110 (2008).

[96] A. R. Rivera, G. A. P. Alcazar, and J. A. Plascak, Diluted and random-bond Ising model: Application to the Fe-Mn-Al alloys. Phys. Rev. B 41, 4774 (1990).

[97] E. Albayrak, Bond Dilution Effects on Bethe Lattice the Spin-1 Blume-Capel Model. Commun. Theor. Phys. 68361 (2017).

[98] M. Hasenbusch, F. Parisen Toldin, A. Pelissetto, and E. Vicari, The universality class of $3 D$ site-diluted and bond-diluted Ising systems. J. Stat. Mech.: Theory Exp. (2007) P02016.

[99] N. G. Fytas and P. E. Theodorakis, Universality in disordered systems: The case of the three-dimensional random-bond Ising model. Phys. Rev. E 82, 062101 (2010).

[100] J. S. Wang, W. Selke, VI. S. Dotsenko,and V. B. Andre-ichenko, The critical behavior of the two-dimensional dilute Ising magnet. Physica A 164, 221 (1990).

[101] H.-O. Heuer, Crossover phenomena in disordered two-dimensional Ising systems: A Monte Carlo study. Phys. Rev. B 45, 5691 (1992).

[102] O. G. Mouritsen and P. J. Shah, Dynamical scaling and crossover from algebraic to logarithmic growth in dilute systems. Phys. Rev. B 40, 11445(R) (1989).

[103] Y. Tomita and Y. Okabe, Crossover and self-averaging in the two-dimensional site-diluted Ising model: Application of probability-changing cluster algorithm. Phys. Rev. E 64, 036114 (2001). 
[104] H. K. Janssen, K. Oerding, and E. Sengespeick, On the crossover to universal criticality in dilute Ising systems. J. Phys. A 28, 6073 (1995).

[105] H. O. Heuer, Critical crossover phenomena in disordered Ising systems. J. Phys. A 26, L333 (1993).

[106] E. Domany, Some results for the two-dimensional Ising model with competing interactions. J. Phys. C 12, L119 (1979).

[107] C. K. Harris and R. B. Stinchcombe, Critical Dynamics at the Percolation Threshold by Fractal and Scaling Approaches. Phys. Rev. Lett. 50, 1399 (1983).

[108] S Jain, Compressive stress enhances coordinated migration of mammary carcinoma cells. J. Phys. A: Math. Gen. 19, L667 (1986).

[109] V. V. Prudnikov and O. N. Markov, Monte Carlo renormalization group of dilute 2D Ising dynamics. EPL, 29, 245 (1995).

[110] G. Parisi, F. Ricci-Tersenghi, and J. J. Ruiz-Lorenzo, Universality in the offequilibrium critical dynamics of the three-dimensional diluted Ising model. Phys. Rev. E 60, 5198 (1999).

[111] D. Ivaneyko, J. Ilnytskyi, B. Berche, and Y. Holovatch, Local and cluster critical dynamics of the $3 \mathrm{~d}$ random-site Ising model. Physica A. 370, 163 (2006).

[112] M. Henkel and M. Pleimling, Superuniversality in phase-ordering disordered ferromagnets. Phys. Rev. B 78, 224419 (2008).

[113] E. Lippiello, A. Mukherhjee, S. Puri, and M. Zannetti, Scaling behavior of response functions in the coarsening dynamics of disordered ferromagnets. EPL 90, 46006 (2010).

[114] K. Binder, Finite size scaling analysis of Ising model block distribution functions. Z. Phys. B 43, 119 (1981).

[115] M. Ohzeki, Locations of multicritical points for spin glasses on regular lattices. Phys. Rev. E. 79, 021129 (2009).

[116] A. B. Harris, Effect of random defects on the critical behavior of Ising models. J. Phys. C: Solid State Phys. 7, 1671 (1974).

[117] F. D. A. Aarão Reis, S. L. A. de Queiroz, and R. R. dos Santos, Universality and logarithmic corrections in two-dimensional random Ising ferromagnets. phys. Rev. B. 56, 6013 (1997).

[118] P. H. L. Martins and J. A. Plascak, Universality class of the two-dimensional site-diluted Ising model. Phys. Rev. E 76, 012102 (2007).

[119] M. Doi, Introduction to Polymer Physics (Oxford University, Oxford, 1996). 
[120] M. Doi and S. F. Edwards, The Theory of Polymer Dynamics (Clarendon Press, Oxford, 1988).

[121] D. Nelson, T. Piran, and S. Weinberg, Statistical Mechanics of Membranes and Surfaces (World Scientific Publishing, Singapore, 2004).

[122] R. Lipowsky and E. Sackmann, Structure and Dymanics of Membranes, Handbook of Biological Physics Vol. 1 (Elsevier Science, Amsterdam, 1995).

[123] C. Picart and D. E. Discher, Actin protofilament orientation at the erythrocyte membrane. Biophys. J. 77, 865 (1999).

[124] E. Sackmann, Biological physics-Origin and perspectives. ChemPhysChem 3, 237 (2002).

[125] T. Hwa, E. Kokufuta, and T. Tanaka, Conformation of graphite oxide membranes in solution. Phys. Rev. A 44, R2235 (1991).

[126] M. S. Spector, E. Naranjo, S. Chiruvolu, and J. A. Zasadzinski, Conformations of a tethered membrane: Crumpling in graphitic oxide? Phys. Rev. Lett. 73, 2867 (1994).

[127] X. Wen et. al., Crumpled and collapsed conformation in graphite oxide membranes. Nature 355, 426 (1992).

[128] G. T. Barkema, D. Panja and J. M. J. van Leeuwen, Structural modes of a polymer in the repton model. J. Chem. Phys. 134, 154901 (2011).

[129] T. Kreer,J. Baschnagel, M. Muller, and K. Binder, Monte Carlo simulation of long chain polymer melts: Crossover from Rouse to reptation dynamics. Macromolecules 34, 1105 (2001).

[130] J. T. Kalathi, S. K. Kumar, M. Rubinstein, and G. S. Grest, Rouse mode analysis of chain relaxation in polymer nanocomposites. Soft Matter, 11, 4123 (2015).

[131] J. T. Kalathi, S. K. Kumar, M. Rubinstein, and G. S. Grest, Rouse mode analysis of chain relaxation in homopolymer melts. Macromolecules 47, 6925 (2014).

[132] B. I. Halperin, P. C. Hohenberg, and S. K. Ma, Renormalization-group methods for critical dynamics: I. Recursion relations and effects of energy conservation. Phys. Rev. B 10, 139 (1974).

[133] M. C. Yalabik, J. D. Gunton, Monte Carlo renormalization-group studies of kinetic Ising models. Phys. Rev. B 25, 534(R) (1982).

[134] F. J. Alexander, D. A. Huse, S. A. Janowsky, Dynamical scaling and decay of correlations for spinodal decomposition at $T_{c}$. Phys. Rev. B 50, 663 (1994).

[135] C. Bechinger, et al. Active particles in complex and crowded environments. Rev. Mod. Phys. 88, 045006 (2016). 
[136] P. Romanczuk, et al. Active brownian particles. The Eur. Phys. J. Spec. Top. 202, 1 (2012).

[137] J. T. Siebert, et al. Critical behavior of active Brownian particles. Phys. Rev. E 98, 030601 (2018).

[138] B. Partridge and C. F. Lee. Critical motility-induced phase separation belongs to the Ising universality class. Phys. Rev. Lett. 123068002 (2019). 


\section{Samenvatting}

Anomale diffusie is een bekend fenomeen in natuurkunde, biologie en vele andere velden van de wetenschap. Echter, dit fenomeen is pas recentelijk gevonden in het 2D Ising model op de kritische temperatuur [28]. In dit proefschrift hebben we gevonden dat anomale diffusie een veelvoorkomend fenomeen is in Ising-achtige modellen op het kritische punt. De modellen besproken in dit proefschrift zijn het normale Ising model, het $2 D$ scalaire $\phi^{4}$ model, en het bond-diluted Ising model.

In hoofdstuk 2 vinden we dat de anomale diffusie een veelvoorkomend fenomeen is op het kritische punt voor 2D en 3D Ising modellen. Door de snelheid autocorrelatie functie van de magnetisatie en de autocorrelatie van de herstelkracht te berekenen, bewijzen we numeriek dat de GLE een toepasbaar model is om anomale diffusie te beschrijven in het Ising model. Bovendien vinden we dat de anomale exponent gebruikt kan worden om de dynamische exponent voor Ising-achtige modellen te bepalen.

We breiden onze vindingen uit naar akritische temperaturen in het Ising model, in hoofdstuk 3. De anomale exponent stroming, i.e., $\dot{c}$ als functie van $c$, is gemeten, en de exacte exponent is gedefinieerd als $\dot{c} \equiv 0$. Omdat we het op een lagere temperatuur dan $T_{c}$ hebben gevonden, kunnen we een exacte exponent vinden voor elke temperatuur, terwijl bij $T<T_{c}$ er geen exacte exponent maar alleen een piekwaarde voor elke temperatuur is. Rondom de kritische temperatuur, stromen de exacte exponenten en de piekwaarden weg van de kritische anomale exponent, wat extra inzichten in het begrijpen van fasetransities biedt.

Vervolgens claimen we dat de anomale exponent gebruikt kan worden als een methode om de dynamische exponent te bepalen. We testen dit idee in het $2 D$ scalaire $\phi^{4}$ model (hoofdstuk 4 ) en het $2 D$ bond-diluted Ising model (hoofdstuk 5). In het $2 D$ scalaire $\phi^{4}$ model, bevestigt de gevonden dynamische exponent dat het model tot de $2 D$ Ising universality klasse behoort. In het $2 D$ bond-diluted Ising model, vinden we dat als de bond concentratie de percolatiegrens benadert, de dynamische exponent stijgt naar oneindig. Dat betekent dat het systeem trager en trager wordt, een fenomeen dat "super slowing down" genoemd wordt.

$\mathrm{Na}$ het numeriek bewijzen en bepalen van verschillende dynamische eigenschap- 
pen, concentreren we ons op het vinden van een manier om deze eigenschappen analytisch te berekenen. Het $2 D$ Ising model met Kawasaki dynamica is bestudeerd in hoofdstuk 6. We laten zien dat de Fourier modi van de algehele magnetisatie een goede benadering zijn van de dynamische eigenmodi. De MSD en de autocorrelatie functie van de magnetisatie zijn afgeleid van de benaderde dynamische eigenmodi. De analytische resultaten fitten de numerieke resultaten vrij goed. Het verkleint ook de impact van finite-size effects, i.e., we kunnen nu resultaten hebben voor grote systeemgrootten met de analytische methode, wat ons meer nauwkeuriger schattingen van de anomale exponent en andere dynamische eigenschappen biedt.

Tot op heden hebben we de anomale diffusie in verschillende Ising-achtige modellen bestudeerd. Er is nog echter veel onontgonnen terrein in het veld van het combineren van anomale diffusie en fasetransities.

In de toekomst willen we ingaan op de vraag of anomale diffusie van de ordeparameter kan worden gevonden op het kritische punt in andere systemen met een tweede-orde fasetransitie. In het bijzonder zijn we geïnteresseerd in het uitbreiden van onze resultaten naar glasstaten. Er zijn nog veel vragen in systemen die glastransitie ondergaan, en we hopen dat de dynamische eigenschappen zoals de mean-square displacement van de ordeparameter nuttige informatie kan bieden, dat ons begrip van glastransities kan verdiepen.

Tenslotte, het fantastische veld van active matter krijgt veel aandacht in de laatste paar jaar [135]. Een van de beroemde modellen is het active Brownian particles (APBs) [136] model, wat een gas-vloeistof achtig transitiediagram heeft. Echter, op het kritische punt is het lastig om nauwkeurige evenwichtseigenschappen te verkrijgen. De precieze positie van het kritische punt is zelfs nog niet duidelijk. Of het kritische gedrag op het kritische punt van de ABPs wel of niet tot de Ising universality klasse behoort, wordt bovendien nog steeds bediscussieerd [137, 138]. Wij vermoeden dat de dynamische eigenschappen meer overtuigend bewijs aanleveren om dit debat op te lossen. Dit idee zal later worden getest. 


\section{Acknowledgements}

Time flies so fast! Until recently, I realized that the ending of the journey of my $\mathrm{PhD}$ is coming. Four years ago, when I arrived in the Netherlands, everything was new to me. I came with excitement, curiosity, and a fear of the unknown. While four years later, I loved the country and the people there and felt that I am so grateful to all the people I have met during the past four years.

First, I would like to thank my supervisors, Gerard and Deb. Thanks a lot, Gerard. Thank you for providing me the chance to experience the adventure with you in the last four years. I learned a lot from Deb and you, not only about how to do research but also how to be a better person. I miss all the discussion with you. The knowledge which I learned from you will stay with me for the rest of my life. Deb, thanks a lot for your patience. Without you and Gerard, I cannot publish those nice papers and graduate so smoothly. I feel that I am so lucky to have you as my daily supervisor. I learned how to do research step by step. Although I know I failed you sometimes, especially in writing.

Second, I want to express my thanks to my collaborator, Robin. I appreciate the discussions with you and your help in our papers. I benefit a lot from your deep insight into the problems we were dealing with.

Besides, I am indebted to many of my colleagues. Sjoerd and Sandeep, thank you for helping me went through the first few months of my PhD. When I was mixed-up about the future and worried about my research, your support on computer programming and explaining the research problems really helps a lot. Federico, it is my honor to work with you in the last four years. The BBQs, each coffee break, lunch, and dinner talks with you are my good memories. Without you, I will feel much lonely. Mark, I am so happy that we can have you join our small group. You are always willing to provide your help and useful advice. Thank you for helping me translate the English summary of my thesis into Dutch. It is not an easy work. Also many thanks to Jolein and Federica for those nice dinners and talks. Zihua, we are so happy that you became a member of our group, although this year is not a normal year because of the virus.

Next, I would like to show my gratitude to many of my friends. Leihua and Jun Liang, you helped us a lot in our daily life. Without your help, life will be much more 
difficult for us. Lianci, you are really a perfect roommate. It is our pleasure to live with you. I enjoy the trips and dinners with you. Yu Yang, my friend, cheers that you find a good job. You were always our driver during our trips, and I am so happy to sit next to you and be your navigator. Chen Lian and Haiping, I realize that I need dozens of pages to fill in the memories we had with you. I appreciate those dinner times, those wonderful discussions, those drinks, and those travels with you. Yuanshuai, Lily and little Shuqiao, thank you for bringing us those memorable moments. All of you, together with Chen, Haiping, Jinghan and myself, we had amazing times on Texel. Jin Ma and Linlin, I am happy that we had those nice discussions and dinners and good luck to both of you. There are so many other friends that I would like to thank, Jiansen, Xiaoqing, Luora, Wentao, Yongtao, Tao Ku, Jeroen, Tianyu, and those names that are not listed here.

I also wish to thank some of my 'old' friends. Weijun and Xiaojun, we are happy that you came to visit us in the Netherlands. Even today, I am still wondering it was a dream or not. Yongkai or I would like to call you 'Zhu Ge'. We don't meet and talk with each other as often as we did. However, you still helped me a lot. Ruofei (Lao $\mathrm{Xu}$ ), I enjoy the project we had together. To create new artwork is never easy, and you did really good job. I also wish to thank Xia Zhang, Hao Zhang, Xinxin Lu, and many other friends and colleagues that I did not mention here. Thank you for each of you.

Last but not least, I would like to express my thankfulness to my parents, my brother and sister, my nieces and other relatives. Most importantly, I want to thank my wife, Ms. Jinghan Zhang. Thank you, my dear. Without your accompany and support, life will be gloomy and boring. You are everything to me. Love you so so much! 


\section{Curriculum Vitae}

The author was born on September 19, 1990, in Changting county, which is a beautiful small city located in the southeast China, Fujian Province. After finishing high school in 2009, the author went to Tianjin, which is a city near Beijing and about 2200 kilometers far away from his hometown, to pursue his bachelor degree in physics. He got the degree from Tianjin Normal University in June 2013, and in that September, he joined Hunan Normal University to continue his master in theoretical physics, under the supervision of Prof. C. M. Xiao. It was a wonderful three years time period. In the summer of 2016, he married his wife Ms. Jinghan Zhang. It was the time for him to decide whether he would like to continue in staying in academia or not, and luckily, he obtained the chance to study computational physics at Utrecht university, beginning in November 2016. In Utrecht, he worked with Prof. Gerard. T. Barkema and Dr. Deb Panja at the department of information and computing sciences, where his research outputs are included in this thesis. 